Portland State University

PDXScholar

1989

\title{
Ultrashort Pulse Production in Synchronously Pumped Mode-Locked Dye Laser Systems
}

Duncan Leo MacFarlane

Portland State University

Follow this and additional works at: https://pdxscholar.library.pdx.edu/open_access_etds

Part of the Electrical and Computer Engineering Commons Let us know how access to this document benefits you.

Recommended Citation

MacFarlane, Duncan Leo, "Ultrashort Pulse Production in Synchronously Pumped Mode-Locked Dye Laser Systems" (1989). Dissertations and Theses. Paper 1392.

https://doi.org/10.15760/etd.1391

This Dissertation is brought to you for free and open access. It has been accepted for inclusion in Dissertations and Theses by an authorized administrator of PDXScholar. Please contact us if we can make this document more accessible: pdxscholar@pdx.edu. 


\title{
ULTRASHORT PULSE PRODUCTION IN SYNCHRONOUSLY PUMPED MODE-LOCRED DYE LASER SYSTEMS
}

by

DUNCAN LEO MACFARLANE

A thesis submitted in partial fulfillment of the requirements for the degree of

\author{
DOCTOR OF PHILOSOPHY \\ in \\ ELECTRICAL ENGINEERING
}

Portland State University

- 1989 
TO THE OFFICE OF GRADUATE STUDIES:

The members of the committee approve the dissertation of Duncan Leo MacFarlane presented March 31, 1989.

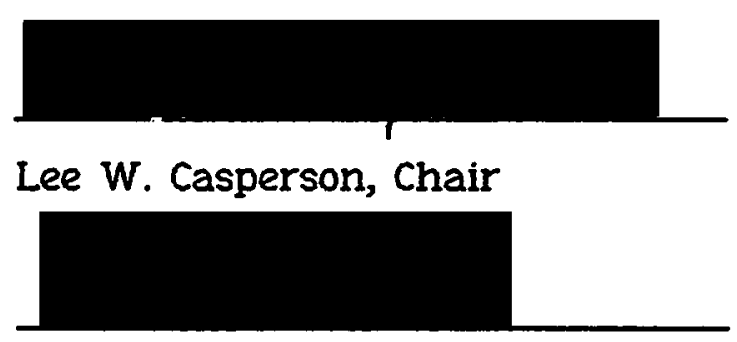

Faris Badi'i
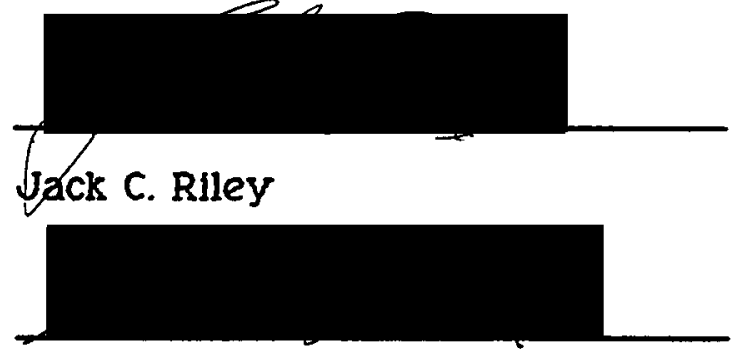

David Paul

APPROVED:

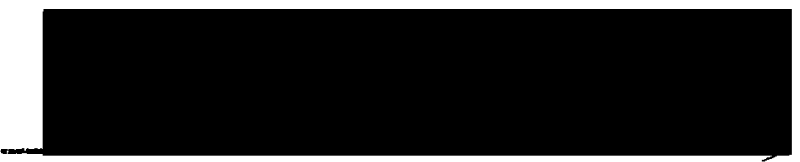

Rolf Schaumann, Head, Department of Electrical Engineering

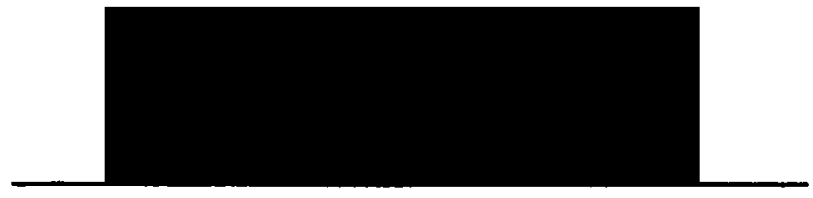

Bernard Ross, Vice Provost for Graduate Studies 
AN ABSTRACT OF THE DISSERTATION OF Duncan Leo MacFarlane for the Doctor of Philosophy in Electrical Engineering presented March 31, 1989.

Title: Ultrashort Pulse Production in Synchronously Pumped Mode-Locked Dye Laser Systems

APPROVED BY THE MEMBERS OF THE DISSERTATION COMMITTEE:

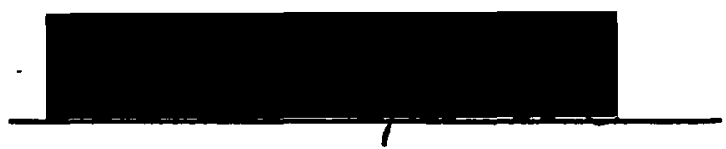

Lee W. Casperson, Chair

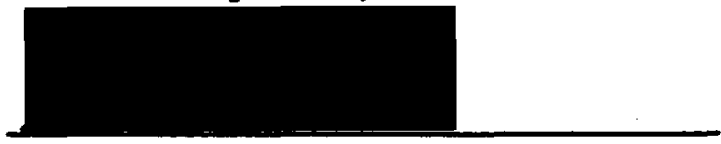

Faris Badi'i

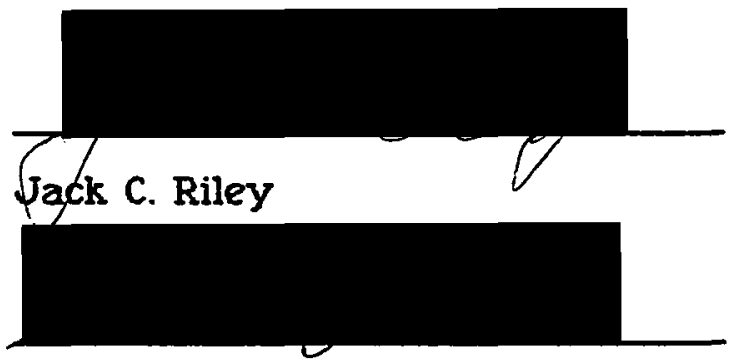

David Paul

The concern of this dissertation is the understanding and improvement of a class of lasers that is responsible for some of the shortest optical pulses available today. In particular, we seek ways to produce from synchronously pumped mode-locked dye laser systems, shorter pulses of higher intensity with improved pulse-to-pulse consistency. Specific topics that are discussed herein 
include the study of the role of the pump pulse in synchronously pumped mode-locked lasers, the study of the pulse shaping and shortening due to an intracavity saturable absorber, and the study of a fundamental pulse train instability associated with these lasers. 


\section{ACKNOWLEDGEMENT}

I have had the good fortune to have interacted with a large number of intelligent people, from whom I have learned much, and whose contributions are, though sometimes subtle, significant.

I must, in particular, thank Professor Lee W. Casperson, who, among many things, taught me to be patient. 1 must also thank Professor Nabil M. Lawandy, who, above all else, taught me to be impatient. To a young scientist today, neither of these skills is more important than the other.

A special thanks, too, goes to my parents for their unwaning support. I am satisfied on those occasions when I make them proud. 
TAELE OF CONTENTS

PAGE

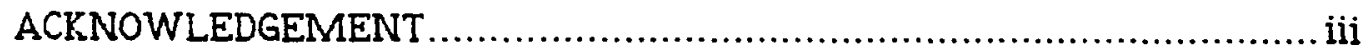

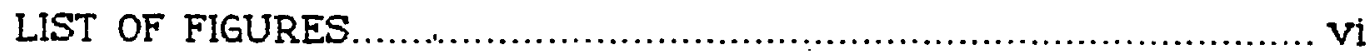

CHAPTER

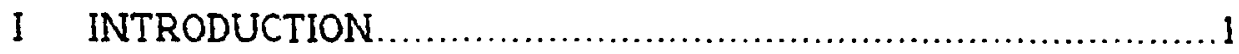

II PUMP PULSE EFFECTS IN SYNCHRONOUSLY

PUMPED MODE-LOCKED DYE LASERS ............................ 4

Introduction....................................................... 4

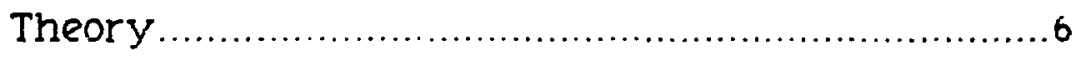

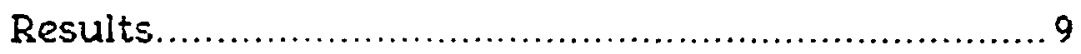

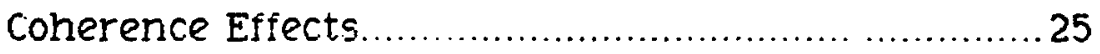

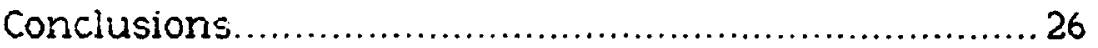

III THEORY OF A SYNCHRONOUSLY PUMPED

MODE-LOCKED DYE LASER IN THE SHORT

PUMP PULSE LIMIT .................................................. 30

Introduction ............................................................ 30

Review and Initial Simplifications

of the Model..................................................... 32

Rate Equation Limit............................................ 39

Zero Detuning Limit.................................... 45

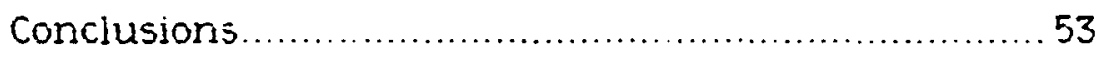


IV COHERENCE EFFECTS IN A HYBRIDLY MODE-LOCKED DYE LASER: THEORY AND EXPERIMENT ..................... 55

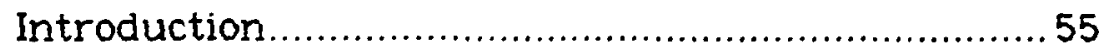

Semiclassical Model .................................................. 58

Density Matrix Equations.................................... 59

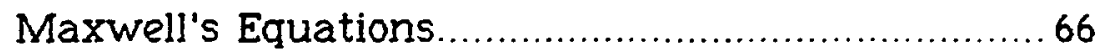

Simplifications and Specializations.........................67

Synchronous Pumping ......................................... 74

Parameter Values................................................... 76

Experimental Results ...........................................79

Theoretical Results................................................... 84

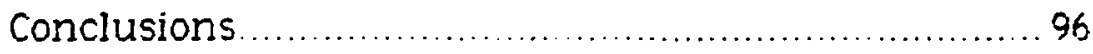

$\mathrm{V}$ SPECTRAL BEHAVIOR AND PULSE TRAIN INSTABILITIES OF A SYNCHRONOUSLY PUMPED MODE-LOCKED DYE LASER ....................................... 102

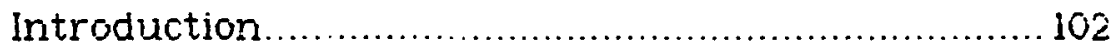

Experimental Setup ...................................... 104

Time and rf Spectra Data....................................... 108

Spectral Behavior of a free running SPML dye laser..........................................110

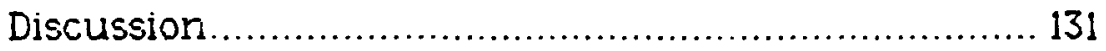

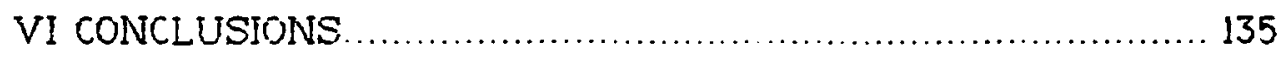

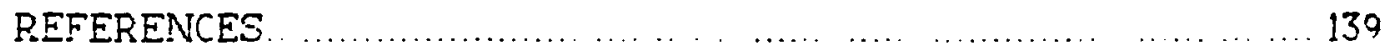

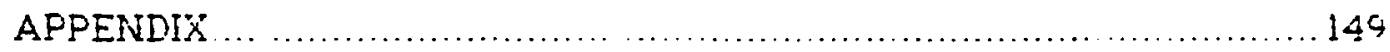




\section{LIST OF FIGURES}

FIGURE

PAGE

1a. SPML pulse width versus the threshold parameter.

1b. SPML pulse width versus the threshold parameter. .12

2a. SPML peak intensity versus the threshold parameter. .13

2b. SPML peak intensity versus the threshold parameter.

3a. SPML occurance time versus the threshold parameter. 15

3b. SPML occurance time versus the threshold parameter......................................16

4. SPML pulse width versus pump pulse width.

5 SPML peak intensity versus pump pulse width

6. SPML occurance time versus pump pulse width. 20

7a. SPMI output pulses for $\Delta \tau=100 \mathrm{psec} \ldots \ldots \ldots \ldots \ldots \ldots . \ldots 21$

7b. SPML output pulses for $\Delta \tau=10 \mathrm{psec} \ldots \ldots \ldots \ldots \ldots \ldots . . \ldots 22$

7c. SPML output pulses for $\Delta \tau=1$ psec.................... 23

8. Error due to the rate equation approximation.........28

9. SPML pulsewidth contours in $r$ and $\Delta \tau$ space .........29 
10. SPML pulse width versus pump

pulse width.

11. SPML output pulse ................................................. 36

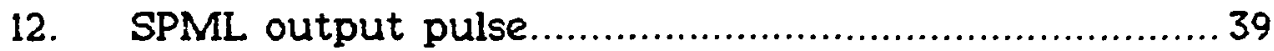

13. SPML peak intensity versus the threshold parameter...........................................41

14. SPMI pulse width versus the threshold parameter. 43

15a. Analytic fit for the SPML output pulse in the rate equation limit

15b. Numerical SPML output pulse in the rate equation limit. 47

16. Analytic solution to the zero cavity length detuning equations. 51

17. Energy level diagram for hybridly mode-locked laser. 60

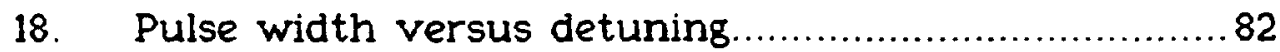

19 Peak intensity versus detuning ............................. 84

20a. Theoretical pulse width versus DODCI molarity. .88

20b. Theoretical pulse width versus DQOCI molarity 89

21a. Theoretical peak intensity versus DODCI molarity ........................................................... 90

21b. Theoretical peak intensity versus DQOCI molarity. 91

22a. Theoretical pulse forms............................................ 93

22b. Theoretical pulse forms........................................... 94

23a. Theoretical pulse width versus $r \ldots \ldots \ldots \ldots \ldots \ldots \ldots \ldots \ldots \ldots . . . \ldots 8$

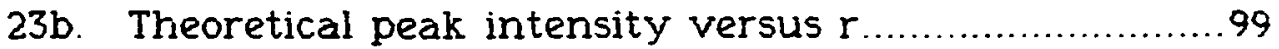


24a. Theoretical pulse width versus pump

pulse width........................................................ 100

24b. Theoretical peak intensity versus pump

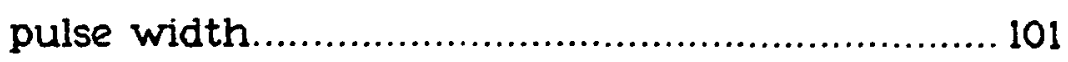

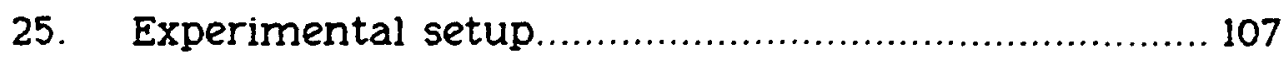

26a. SPML pulse train at optimum cavity length.............112

26b. SPML $r f$ spectrum at optimum cavity length........... 113

27a. SPML pulse train envelope at

$\Delta \mathrm{L}=125$ microns.............................................114

27b. SPML $\mathrm{rf}$ spectrum at $\Delta \mathrm{L}=125$ microns...................115

27c. SPML pulse train envelope at

$\Delta \mathrm{L}=320$ microns............................................ 116

27d. SPML rf spectrum at $\Delta \mathrm{L}=320$ microns....................117

28. Modulation frequency versus detuning ..................... 118

29a. SPML pulse train envelope at

$\Delta \mathrm{L}=-60$ microns............................................ 119

29b. SPML $r$ spectrum at $\Delta L=-60$ microns.................... 120

30a. SPML pulse train envelope at

$\Delta \mathrm{L}=-125$ microns........................................... 121

30b. SPML rf spectrum at $\Delta \mathrm{L}=-125$ microns................... 122

31a. SPML pulse train envelope at

$\Delta \mathrm{L}=-128$ microns....................................... 123

31b. SPML rf spectrum at $\Delta L=-128$ microns................... 124

32. Peak lasing wavelength versus detuning.................. 125

33a. Emission spectrum of SPML laser at

$\Delta \mathrm{L}=-35$ microns.

33b. Emission spectrum of SPIMI laser at

optimum cavity length

33c. Emission spectrum of SPML laser at

$\Delta \mathrm{L}=92$ microns 128 
33d. Emission spectrum of SPML laser at

$$
\Delta L=280 \text { microns..................................... } 129
$$

34. Emission spectrum of SPMI laser..........................130

35a. Sampling scope trace........................................ 156

35b. Sampling scope trace................................... 157

35c. Sampling scope trace ...................................... 158

35d. Sampling scope trace............................................159

36a. Pulse train envelope.......................................... 160

36b. Pulse train envelope........................................... 161

37a. If spectrum.............................................. 162

37b. If spectrum.................................................. 163

38. Average power versus detuning ......................... 164 


\section{CHAPTER I}

\section{INTRODUCTION}

The concern of this dissertation is the understanding and improvement of a class of lasers that is responsible for some of the shortest optical pulses available today, the synchronously pumped mode-locked (SPML) dye laser. Depending on the particular attributes of the system at hand, these lasers produce wavelength tunable pulses in the range of $10^{-14}-10^{-12} \mathrm{sec}$. These ultrashort optical pulses represent a remarkable precision in the localization of energy and information, and thus inspire innovations in the areas of spectroscopy and photochemistry, electronic material and electronic circuit characterization, electro-optic and opto-optic sampling, and optical communication and optical computing. Thus far, ultrashort pulse production has been the most effective method of exploiting the huge bandwidth associated with nearly every laser.

This dissertation seeks ways to obtain improved performance from SPML dye laser systems. Parameters of interest include shorter pulse widths, higher peak intensities and improved pulse-to-pulse consistency. At the same time, an attempt is made herein to provide an intuitive understanding of the operation of SPML lasers, and, through generalization, an intuitive understanding of other mode-locked lasers.

Each chapter of this dissertation is a study complete within itself. It is, however, hoped that each chapter has a non-sibi 
quality about it, and that the juxtaposition of one chapter with respect to the others increases its value. In our first study, entitled, "Pump pulse effects in synchronously pumped mode-locked dye lasers," we look at the optimization of a SPML dye laser through parameters associated with the pump pulse that dynamically drives the SPML laser system. The approach used in this chapter is largely theoretical, however the theoretical results and predictions of this chapter closely agree with the experiments performed to date. One of the important results of this chapter is the discovery of a practical Dirac delta function response limit which represents an optimized point of operation for these lasers; the shortest and most intense output pulses are formed when the pump pulse length is one picosecond or less. Formally and theoretically, this point of operation also represents a great mathematical simplification which is exploited in the next chapter, "Theory of a synchronously pumped mode-locked dye laser in the short pump pulse limit." In this chapter, we analytically explore the theory of operation of a SPML dye laser in the delta function limit. The closed form results obtained here, although highly approximate, do describe the trends and important empirical aspects of a SPML laser. Because these expressions may be written simply in closed form, they provide an immediate intuitive understanding of the operation of these lasers. While these first two studies consider - to some extent the improvement of a SPML laser with respect to the external pump pulse, the third study looks at the dramatic decrease in pulse width attained when a saturable absorber is placed inside the SPML laser cavity. Because mode-locking in this situation occurs by both active and passive methods, this experimental 
configuration is called hybrid mode-locking. Chapter IV, then, is called, "Coherence effects in a hybridly mode-locked dye laser: theory and experiment." The last chapter, "Spectral behavior and pulse train instabilities of a synchronously pumped mode-locked dye laser," contains an experimental study of the pulse-to-pulse stability of a SPML dye laser. It is desirable from the applications standpoint that each pulse from a SPML laser be consistent to within a small error. Our work shows that typical errors are approximately $20 \%$ and are therefore non-negligible. In the appendix, "Pulse train instabilities in a mode-locked argon laser: experimental studies," we study similar effects in the more temperate, loss modulated argon ion laser. When these two studies are taken together, they point out a fundamental instability associated with actively mode-locked lasers. 
CHAPTER II

\section{PUMP PULSE EFFECTS IN SYNCHRONOUSLY PUMPED MODE-LOCKED DYE LASERS}

\section{INTRODUCTION}

The synchronously pumped mode-locked (SPML) dye laser has benefited repeatedly in its 20 year history from improved pump sources. The SPML concept was first demonstrated in 1968 using pulsed pumped sources [1-3], however the technique remained of limited practical value until cw mode-locked pump sources were exploited [4,5]. Recently there have again been significant improvements in available pump sources for SPML dye lasers; notable is the fiber/grating compressed Nd:YAG laser which can deliver subpicosecond pulses [6]. When pulses from these laser systems are doubled and used to synchronously pump an organic dye laser, tunable pulses of 200 - 300 femtoseconds are obtained directly from the SPML laser $[7,8]$. The purpose of this chapter is to support these ongoing technological improvements in SPML pump sources with a sound theoretical discussion on the role of the pump pulse in synchronously pumped, mode-locked dye lasers. Our numerical results are in good agreement with the recent experiments but suggest that only limited further improvements may be possible.

The theoretical understanding of SPML lasers has advanced on both the analytic and the numerical level. Because of the stringent approximations imposed in the analytic theory, the two 
approaches are perhaps best considered as distinct, at least in their goals. The analytic work has evolved from the self-consistent pulse picture of Kuizenga and Siegman [9] and of Haus [10] and specific application of this formalism to SPML systems has been considered by Frigo et. al. [11], Ausschnitt et. al. [12], and Kim et. al. [13]. These studies have provided closed-form, approximate expressions for the output pulse characteristics as functions of cavity detuning, system gain and several dye parameters. More rigorous numerical modeling of SPML dye lasers has been undertaken by many groups [14-25], since only this technique affords the study of transient effects and specific pulse shape information. The numerical models also have the advantage that their framework can easily support the study of random noise sources, such as spontaneous emission which can lead to profound effects at large cavity mismatches, far from the optimum cavity length $[20,21]$. Much of this computational work has been done in the rate equation limit, however semiclassical effects have been shown to be significant in the steady state pulse shape [21-25].

An important point of study for nearly all these references has been the dependence of the SPML laser output on the cavity length mismatch between the pump laser and the SPML laser since this is a strong and sensitive test of a candidate theory and is of great practical import. Found in most practical systems is a bandwidth limiting tuning element and the effects of these filters have been well considered $[11,12,13,16,20,21,25]$ On the other hand, only cursory attention has been paid to the adjustable parameters associated with the pump source. The analysis of Ausschnitt, Jain and Heritage predicts that at zero mismatch the SPML pulse width varies as the square root of the pump pulse width [12]. Kim, Kuhl, Lambrich and von der Linde, in an analysis under 
slightly different conditions, show the leading term of the SPML pulse width to go as the cube root of the pump pulse width [13]. Both these works were highly approximate and there has been no rigorous numerical study detailing pump pulse effects in SPML lasers.

In this chapter we explicitly consider the role of the pump pulse in the output of a SPML dye laser by numerically modeling the rhodamine $6 G$ laser under different pumping conditions. In section 2 of this work we review the theoretical model developed in [24]. This nonlinear dynamical model includes semiclassical effects, dipole orientational effects and intraband relaxation. The results of our calculations are then presented in section 3 . In particular, we find a shortening of the output pulse with decreasing pump pulse length or increasing pump pulse level. The effect of pump pulse width is compared with the experiments of [7] and [8] and the effect of pumping level is tested with our own data. Our theory agrees quantitatively with available experimental data, and we also present results from regimes outside the realm of current experimental interest. In section 4 we reduce our model to the rate equation limit and discuss the importance of coherence effects in ultrashort pulse laser modeling. We present a summary in section 4 and also consider the possibility of further enhancements in SPML lasers.

\section{THEORY}

Our starting point is the semiclassical dye laser amplifier model developed in reference [24]. Begining with the density matrix equations and Maxwell's equations, that paper derived a set of ordinary, nonlinear differential equations that govern the 
interplay of the electric field with the molecular populations and the polarization. When the electric field is normalized to its steady state gain saturating value, this set of equations may be written as:

$\mathrm{dD} / \mathrm{dt}=-1 / \tau_{2}\left[\left(1+\tau_{2} / 2 \tau_{1}\right) \mathrm{D}+\left(1-\tau_{2} / 2 \tau_{1}\right) \mathrm{M}+2 \mathrm{QAx}-\mathrm{Px}^{2}\right]$

$\mathrm{dM} / \mathrm{dt}=-1 / \tau_{2}\left[-\left(\tau_{2} / 2 \tau_{1}\right) D+\left(\tau_{2} / 2 \tau_{1}\right) M-P x^{2}\right]$

$\mathrm{dQ} / \mathrm{dt}=-1 / \mathrm{T}_{\mathrm{S}}[\mathrm{Q}-\mathrm{ADx}]$

$d A / d t=-L / 2 t_{c} \Delta L\left[A-\int_{0}^{1} Q x d x\right]$

In equations (1) - (4), D is a normalized population difference, $M$ is a normalized population sum, $Q$ is a normalized polarization and $A$ is a normalized electric field. The dye decay time from the $S_{1}$ band to the $S_{0}$ band is $\tau_{2}$, and $\tau_{1}$ is the lower manifold intraband vibrational relaxation time. $T_{S}$ is the semiclassical coherence time. In our modeling of the rhodamine $6 G$ laser, $\tau_{1}=1 \mathrm{psec}, \tau_{2}=5 \mathrm{nsec}$, and $T_{S}=50$ fsec. The dynamical rate constant associated with the field is composed of the cavity length, $L$, the cavity lifetime, $t_{c}$ and the optical length mismatch, $\Delta \mathrm{L}$, between the pump laser cavity and that of the dye laser. In this work, $L=1.8 \mathrm{~m}, t_{c}=10$ nsec, and $\Delta \mathrm{L}$ is kept in the $0-30$ micron range that gives the shortest output pulses. This model is also general enough to explicitly include the orientation of the molecular dipoles, $x=\cos (\theta)$, and, in particular, we have chosen an isotropic molecular distribution. Thus equations (1) - (3) refer specifically to only one orientational class. 
The individual classes contribute to the field through the integral over $\mathrm{x}$ in equation (4). Properly accounting for the different classes is important in accurately establishing the pumping threshold level; misaligned electric dipoles represent an ensemble inefficiency. Orientational effects also manifest themselves in the specific output pulse shape, particularly at small $\Delta L$ 's [24].

Of specific interest in this paper is the normalized pump function, $P(t)$ and for most of this work, a Gaussian shape was chosen:

$P(t)=P_{0}\left(2 / \Delta_{\tau}\right)\left(\ln 2 / \tau^{1 / 2} \exp \left[-\left(2 t / \Delta_{\tau}\right)^{2} \ln 2\right]\right.$

Importantly from the point of view of this paper, the pump represents two additional control parameters, the pump pulse width, $\Delta \tau$, and the pump level, $P_{0}$, with which the dye laser output may be optimized.

It was demonstrated in [24] that this set of equations adequately describes the pulse shapes for typical SPML dye laser systems in operation at the time. The main point of comparison in [24] was the sensitive behavior of the satellite pulses as a function of $\Delta \mathrm{L}$, and care was taken to match the experimentally measured autocorrelations with numerically calculated autocorrelations. Missing from our set is a description of the bandwidth limiting filter with which the operating wavelength is tuned, and it is possible that the agreement between our theory and the experiments below might be improved if the effects of such a filter were explicitly included in our modeling. In the next section we present the results of our study, focusing on the importance of $\Delta \tau$ and $P_{0}$ and emphasizing systems of current experimental interest. 
RESULTS

Equations (1) - (5) were numerically solved on a Sun workstation minicomputer using a standard Runge-kutta routine for the time integration and a Simpson's routine for the integral over the dipole orientation. The individual pulse dynamics have, in general, been reported on extensively [14 - 25] and for this study we are more concerned with the trends associated with various pertinent output pulse parameters as either the pump level or the pump pulse width is varied. In particular we concentrate on the full-width at half-maximum (FWHM) pulse width, $t_{p}$, the peak intensity, $I_{p k}$, and the time, $I_{p k}$, at which the peak of the output pulse occurs. $T_{\mathrm{pk}}$ is measured with respect to the Gaussian pump pulse which is centered at zero, and it should therefore be noted that when the dye laser pulse occurs in the first half of the pumping process, $T_{\mathrm{pk}}$ is negative.

In keeping with our own SPML system, an argon ion pumped rhodamine dye laser, and the more recently developed systems in [8] we studied the effects of the pump level on $t_{p}, l_{p k}$, and $T_{p k}$ for pump pulse widths of $100 \mathrm{psec}$ and $10 \mathrm{psec}$. The pump level is given here in units of the threshold parameter, $r$, which is the pump level divided by the threshold pump level. Hence $r=1$ corresponds to the minimum value for lasing.

Figures la and $1 \mathrm{~b}$ show a rapidly decreasing $t_{p}$ as $r$ increases from 1 to 3 . Values of $r=5$ or higher represent a practically unrealizable region, but increases in $r$ beyond values around 4 result in very small decreases in $t_{p}$. The different contours in Figs. 
la and $1 \mathrm{~b}$ correspond to different cavity detunings. The data points in Fig. la were measured using our commercially available Spectra Physics argon ion/rhodamine 66 dye laser system. In these experiments, the threshold parameter was varied by linearly attenuating the argon pump beam and was measured as the ratio of the average power of the data point to the average power that just extinguished the laser. The dye laser pulse width was measured using a Spectra Physics 409 autocorrelator at the cavity length that gave the optimum, shortest pulse, which, as suggested by the theory is around 5 microns.

In Figs $2 \mathrm{a}$ and $2 \mathrm{~b}$ are plots of $\mathrm{I}_{\mathrm{pk}}$ vs. $\mathrm{r}$ for $\Delta \mathrm{t}=100 \mathrm{psec}$ and $\Delta \tau=10$ psec, respectively. Noteworthy is the sharp increase in $\mathrm{l}_{\mathrm{pk}}$ as $r$ increases from 1 to 3 . The scale in $2 \mathrm{~b}$ is 10 times greater than in $2 \mathrm{a}$. As $r$ increases for a given $\Delta \tau$ the output pulse occurs sooner, as shown in Figs. $3 \mathrm{a}$ and $3 \mathrm{~b}$. The zero crossing for $\mathrm{T}_{\mathrm{pk}}$ occurs around $r=3$ for $\Delta \tau=100$ psec and between $r=4-8$ for $\Delta \tau=$ 10 psec.

Of perhaps more interest is the variation in $t_{p}, I_{p k}$, and $T_{p k}$ as functions of $\Delta \tau$. Figure 4 is a semilog plot of of $t_{p}$ vs $\Delta \tau$ for various values of $r$ and $\Delta L$. Superimposed on the calculated curves are data taken from references [7] and [8]. While this graph shows significant improvement in pulse width when $\Delta t$ decreases from 100 psec to $1 \mathrm{psec}$, there is no significant improvement upon further shortening, even to 1 fsec. When the midrange values $(\Delta \tau$ $=500$ psec to 1 psec) are fitted to the relation:

$$
t_{p}=\beta \Delta \tau^{a}
$$




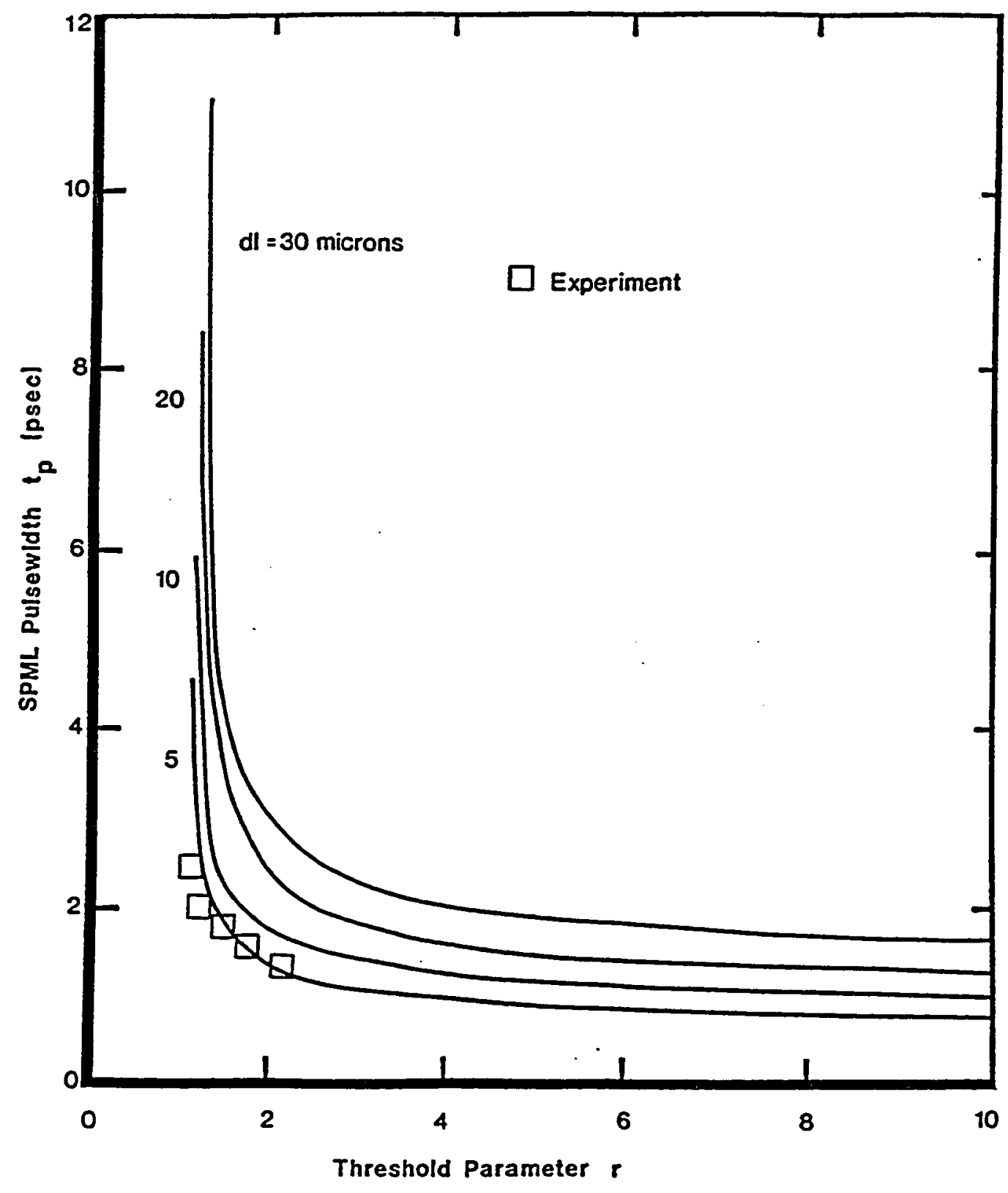

Figure 1a. SPML pulse width versus the threshold parameter. $\quad \Delta i=100$ psec. 


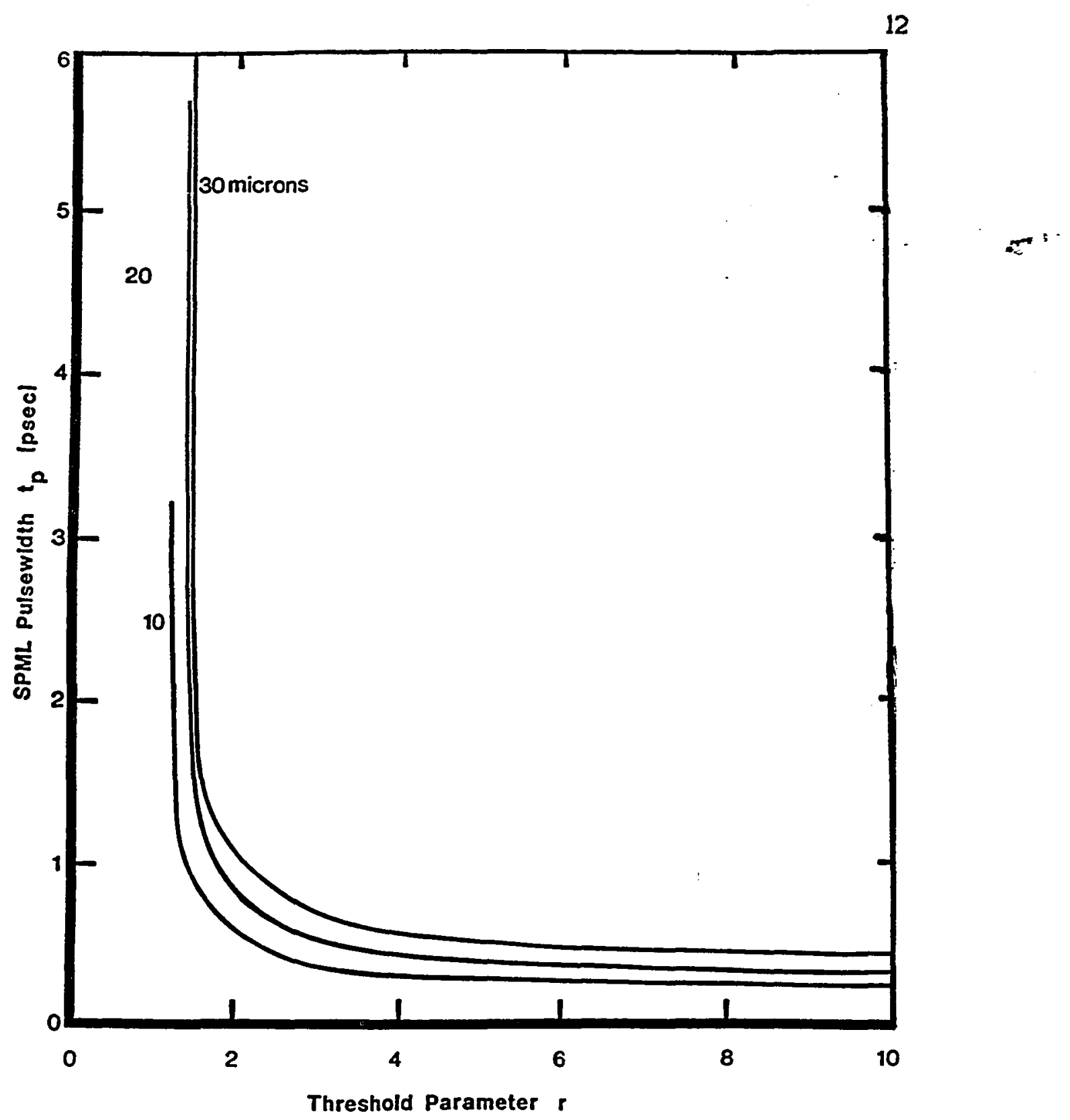

Figure 1b. SPML pulse width versus the threshold parameter. $\Delta r=10 \mathrm{psec}$. 


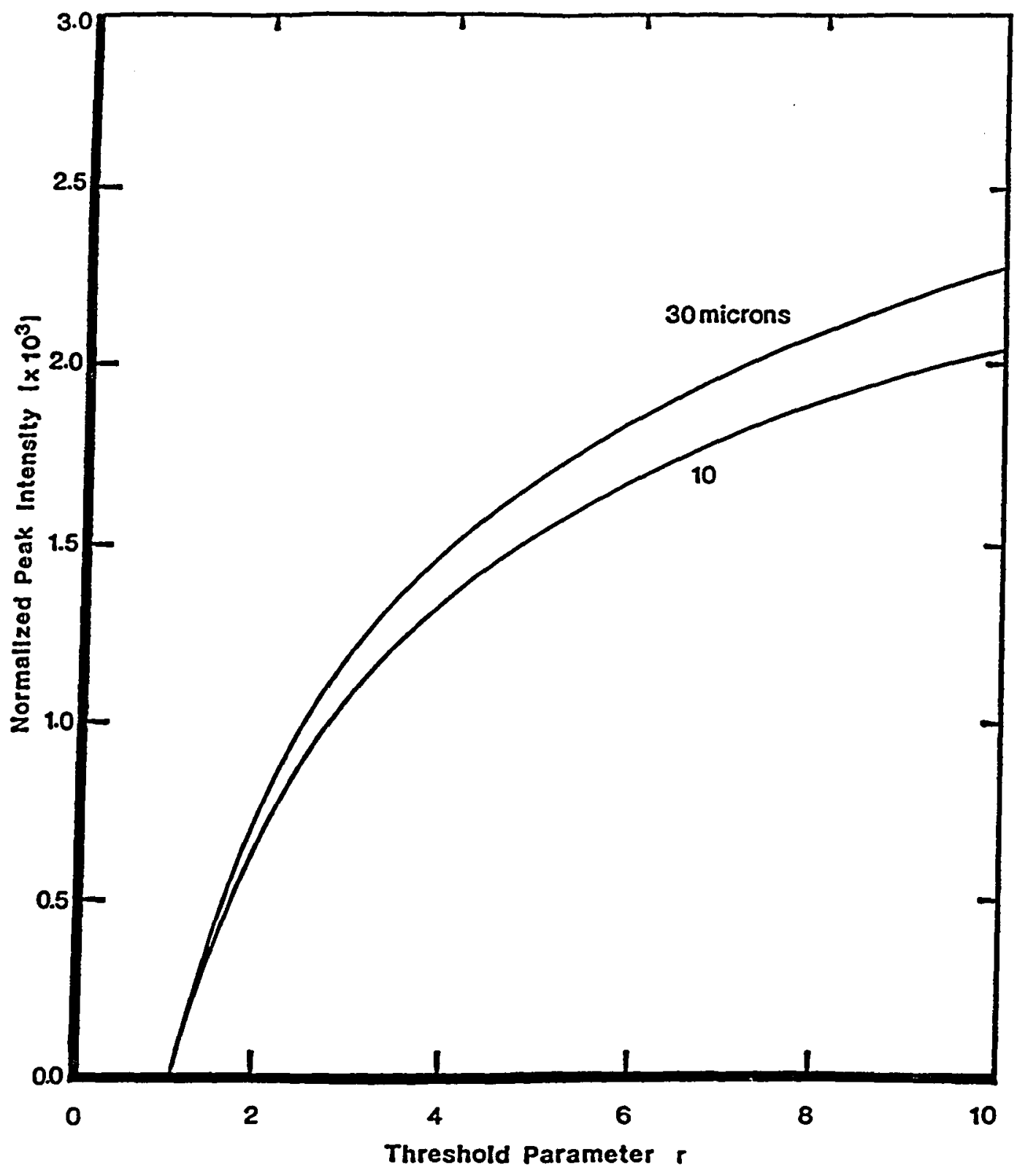

Figure 2a. SPML peak intensity versus the threshold parameter. $\quad \Delta t=100$ psec. 


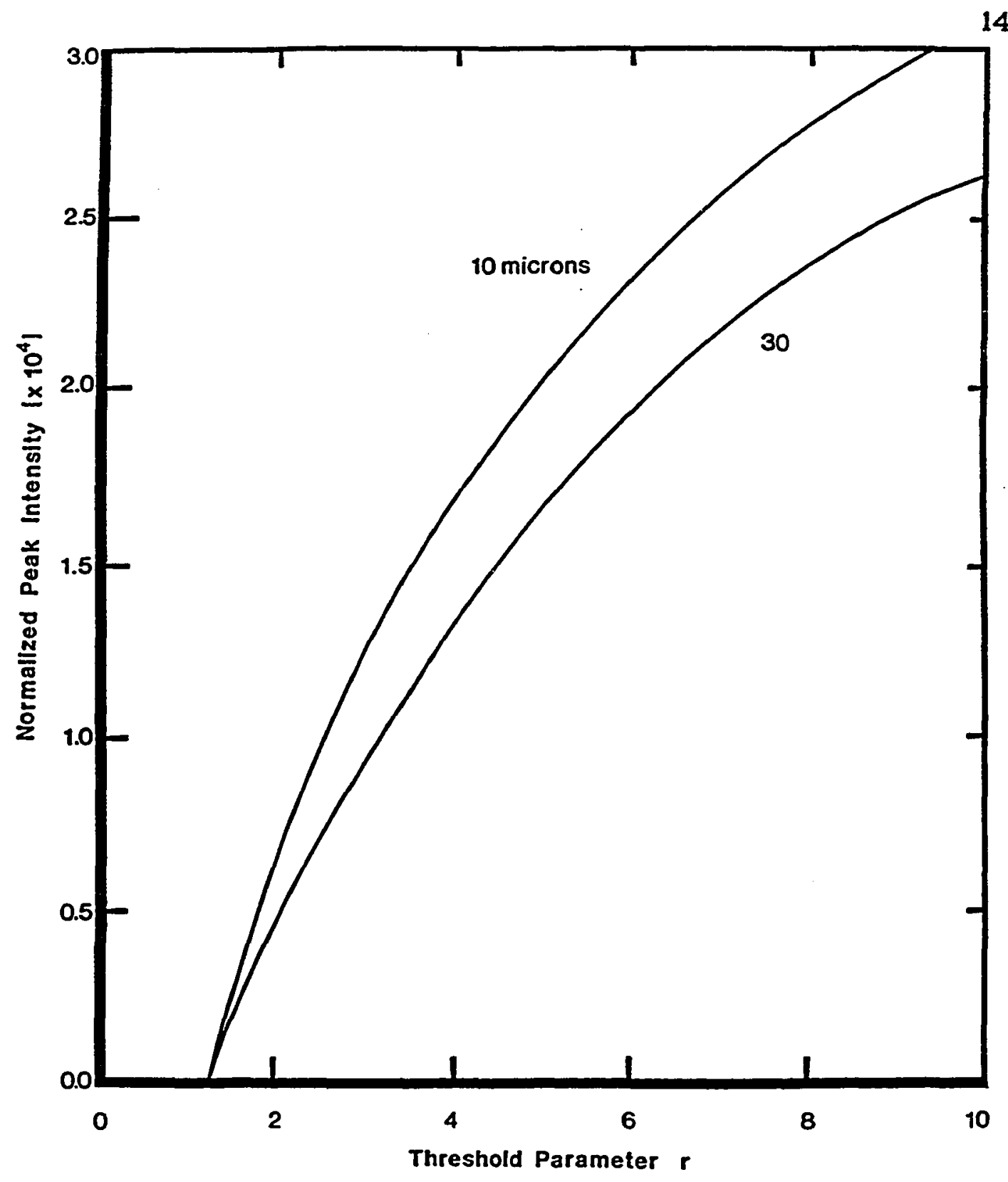

Figure 2b. SPML peak intensity versus the threshold parameter. $\quad \Delta \mathrm{r}=10 \mathrm{psec}$. 


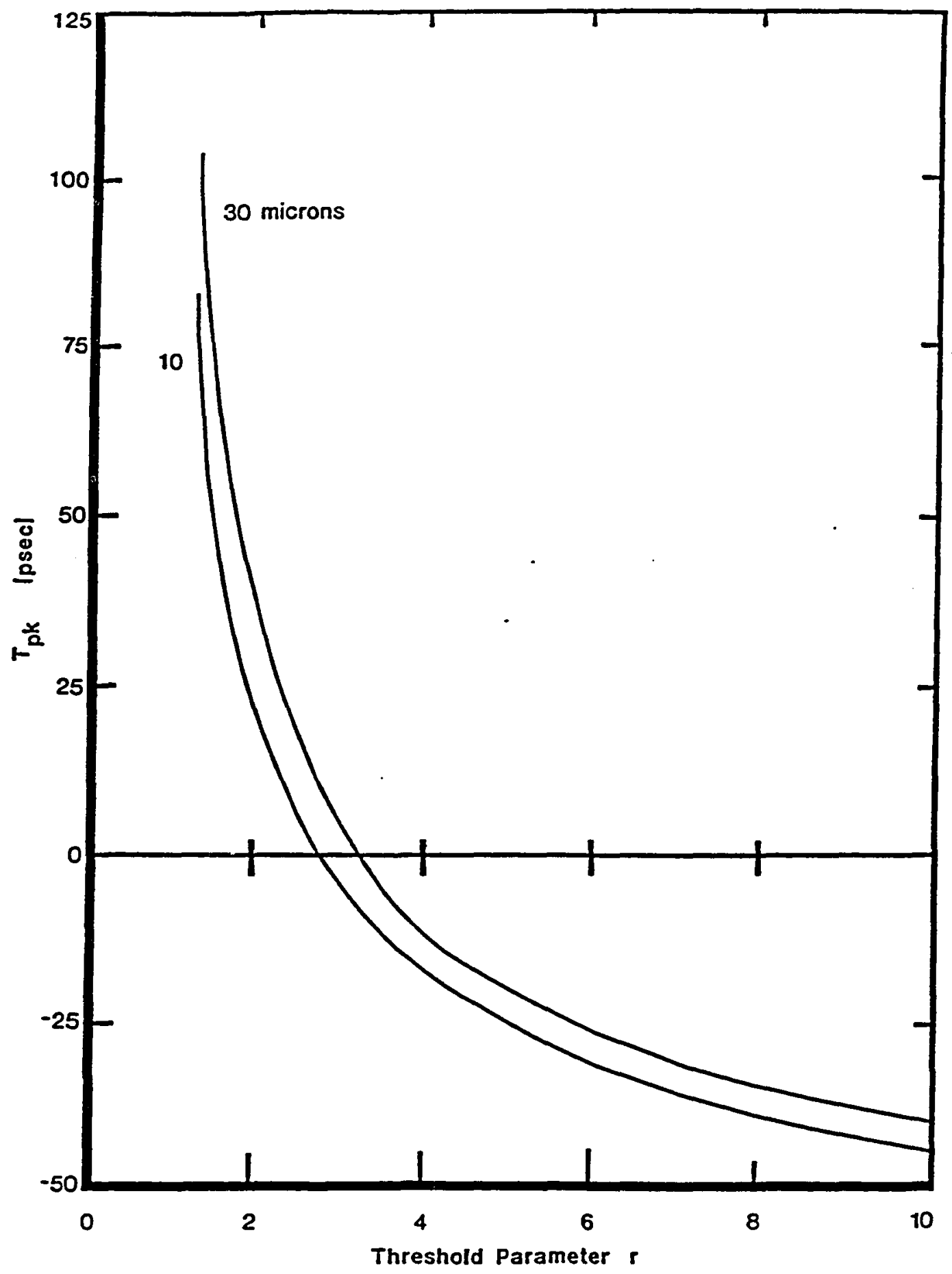

Figure 3a. SPML occurance time versus the threshold parameter. $\quad \Delta \mathrm{r}=100$ psec. 


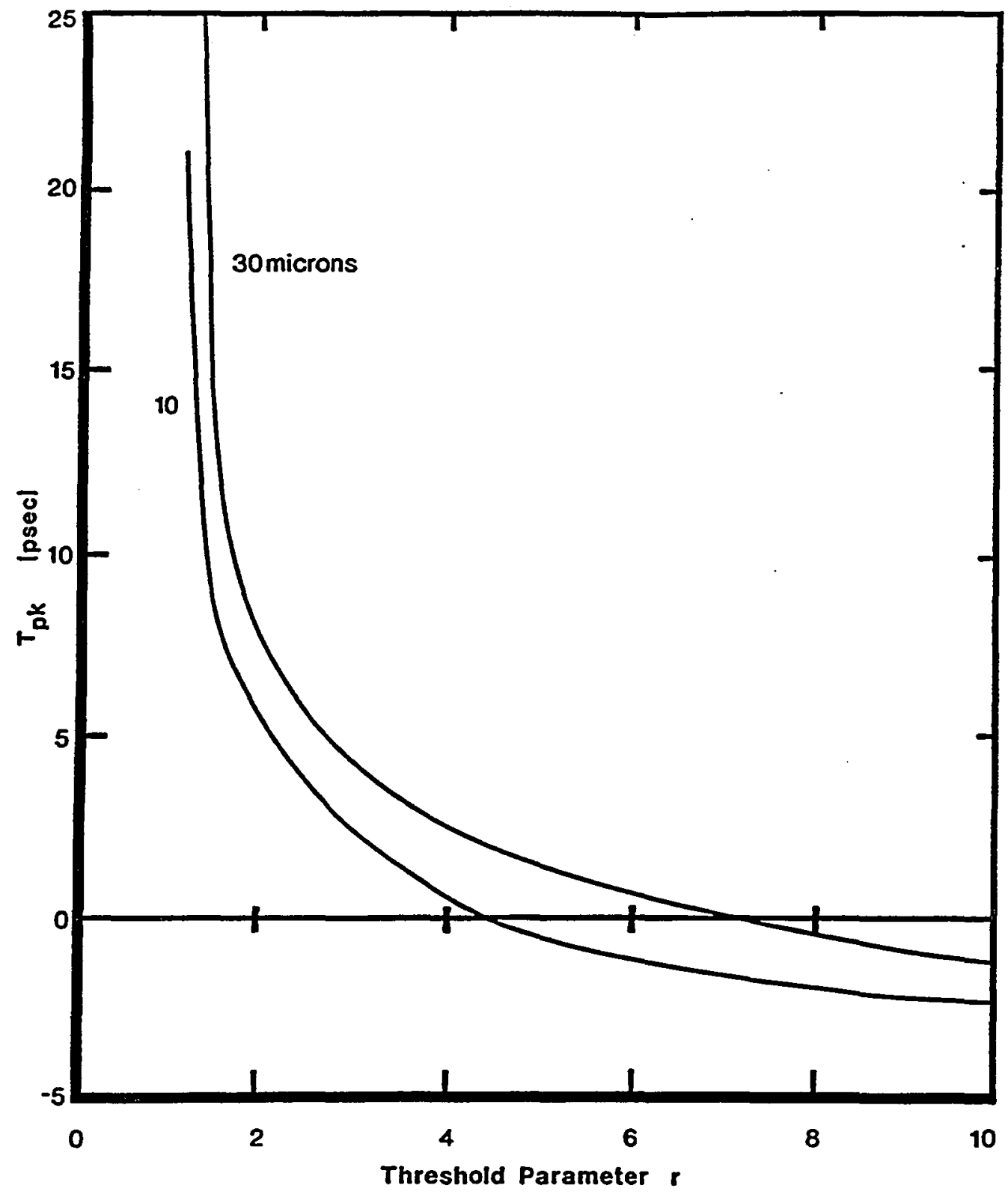

Figure $3 b$. SPML occurance time versus the threshold parameter. $\Delta t=10$ psec. 


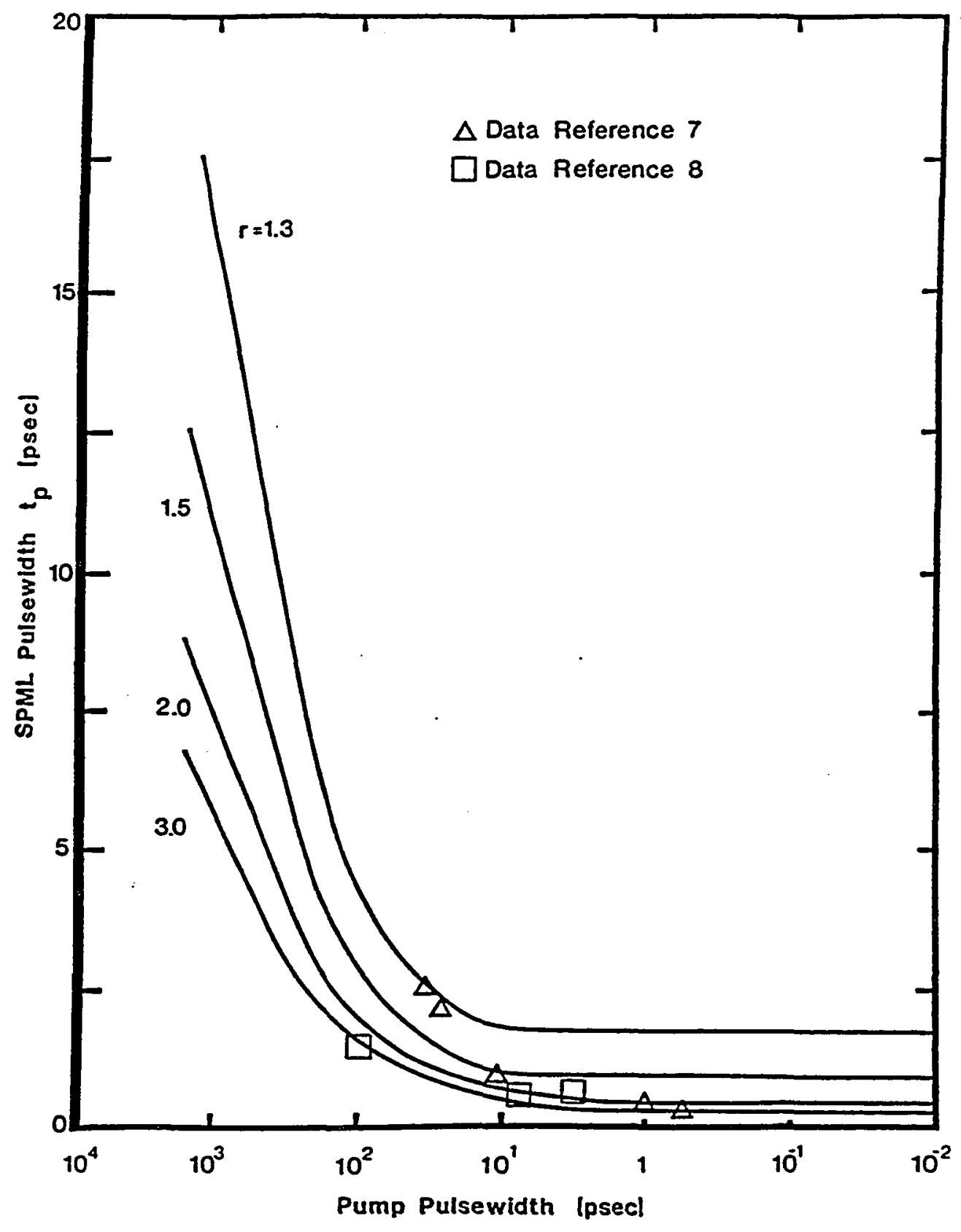

Figure 4. SPML pulse width versus pump pulse width. 
an about $2 / 5$ is typically calculated. This is in fair agreement with the 1/2 value derived by Ausschnitt, Jain and Heritage in [12] and the 1/3 value derived by $\mathrm{Kim}$ et. al. in [13].

Figure 5 shows the character of $I_{\mathrm{pk}}$ as $\Delta \tau$ is shortened. Of practical interest is the large increase in peak intensity predicted if $r$ is increased to 3 times above threshold and $\Delta \tau$ is decreased to $1 \mathrm{psec}$, but no improvement in $I_{\mathrm{pk}}$ occurs when $\Delta x$ is shortened further. The pump pulse delay, $T_{p k}$, as a function of $\Delta \tau$ is shown in Fig. 6 for several values of $r$ and $\Delta L$. It is noteworthy that each curve approaches a constant, $T_{\mathrm{pk}}=2.5 \mathrm{psec}$ for $\Delta \mathrm{\tau}$ 's shorter than a few psec. Thus, for subpicosecond pump pulses, the dye laser pulse is formed after the completion of the pump.

This limit where the pulse width, $t_{p}$, the peak intensity, $I_{p k}$, and the pulse peak time, $T_{p k}$, become insensitive to further decreases in $\Delta t$ is clearly the delta function limit. For the cases chosen here, this limit is approximately $\Delta \tau=1$ psec.

It is of interest to also study pulse shaping as a function of pump pulse length, and plots of calculated output pulses for fixed $\mathrm{r}=3$ and $\Delta \mathrm{L}=10$ microns are shown in Figs. $7 \mathrm{a}, \mathrm{b}$ and $\mathrm{c}$ for $\Delta \mathrm{t}=$ 100 psec, $10 \mathrm{psec}$ and $1 \mathrm{psec}$ respectively. Importantly, the satellite pulses diminish as $\Delta \tau$ decreases. This is not surprising since the cause of the satellite pulses is principally a surplus of gain delivered by the pump pulse after the primary output pulse is formed [24]. Near the delta function limit, all the gain is delivered before dye laser action starts, and only one pulse is formed. We note that the published autocorrelation in [8] shows no satellite 


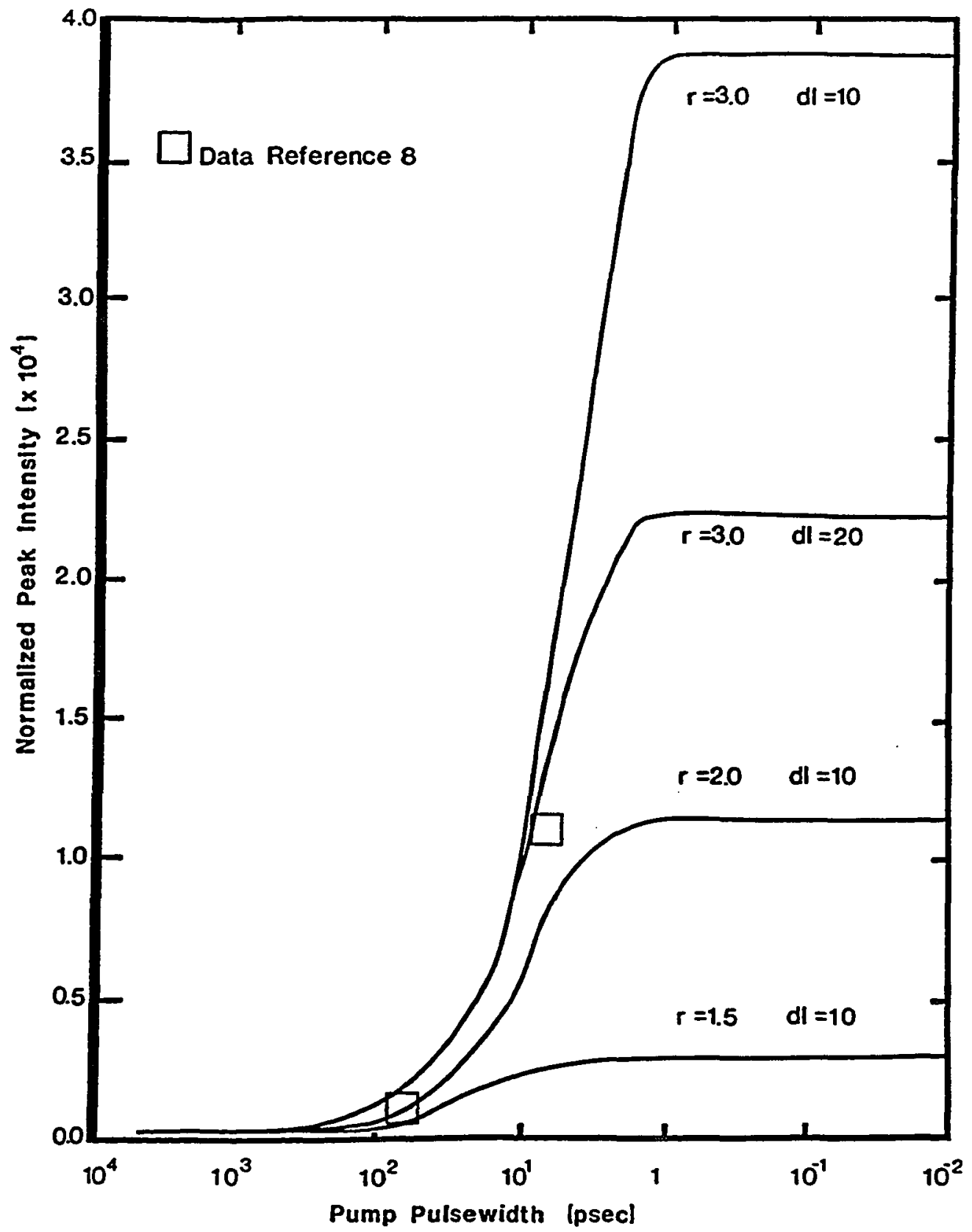

Figure 5. SPML peak intensity versus pump pulse width. 


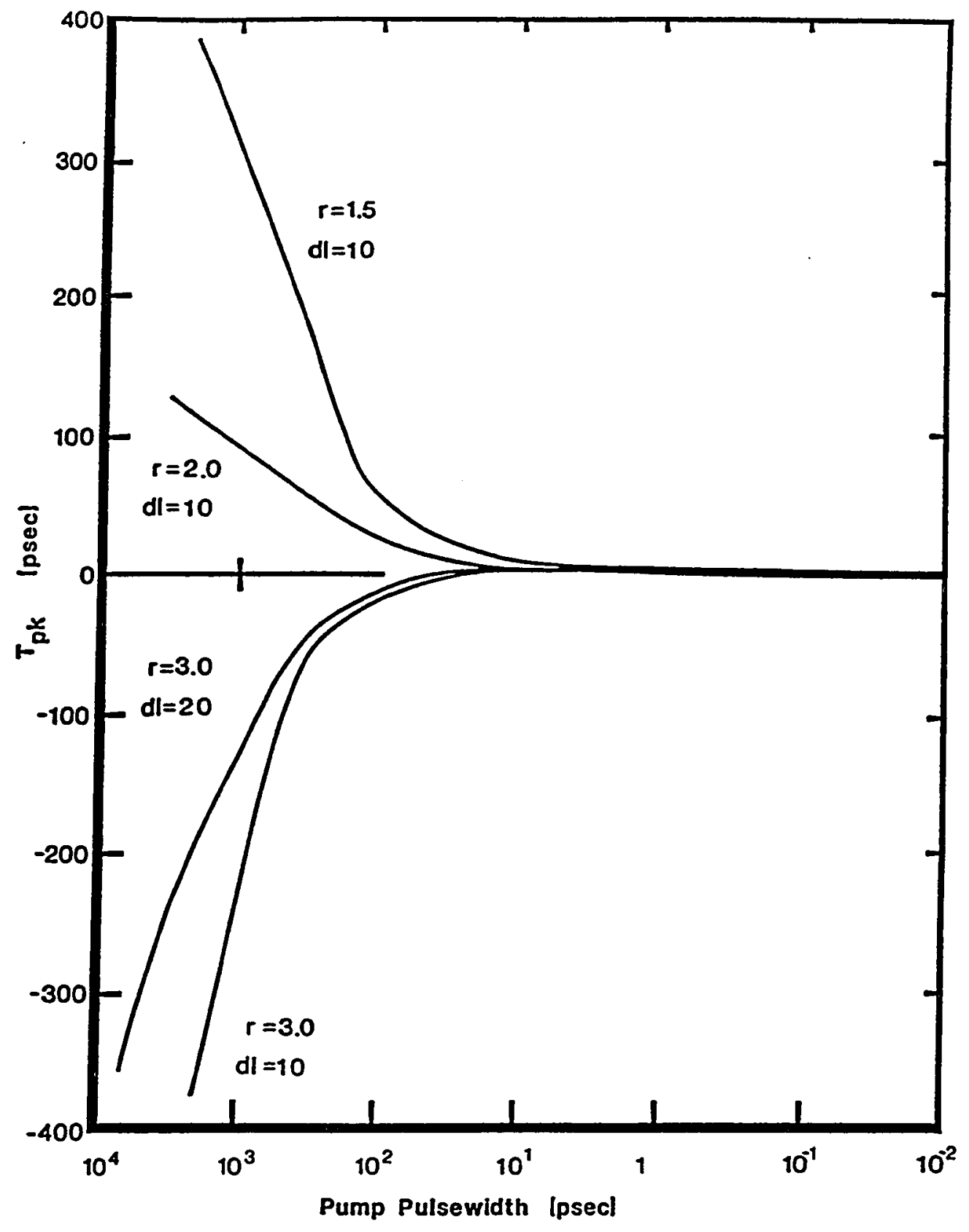

Figure 6. SPML occurance time versus pump pulse width. 


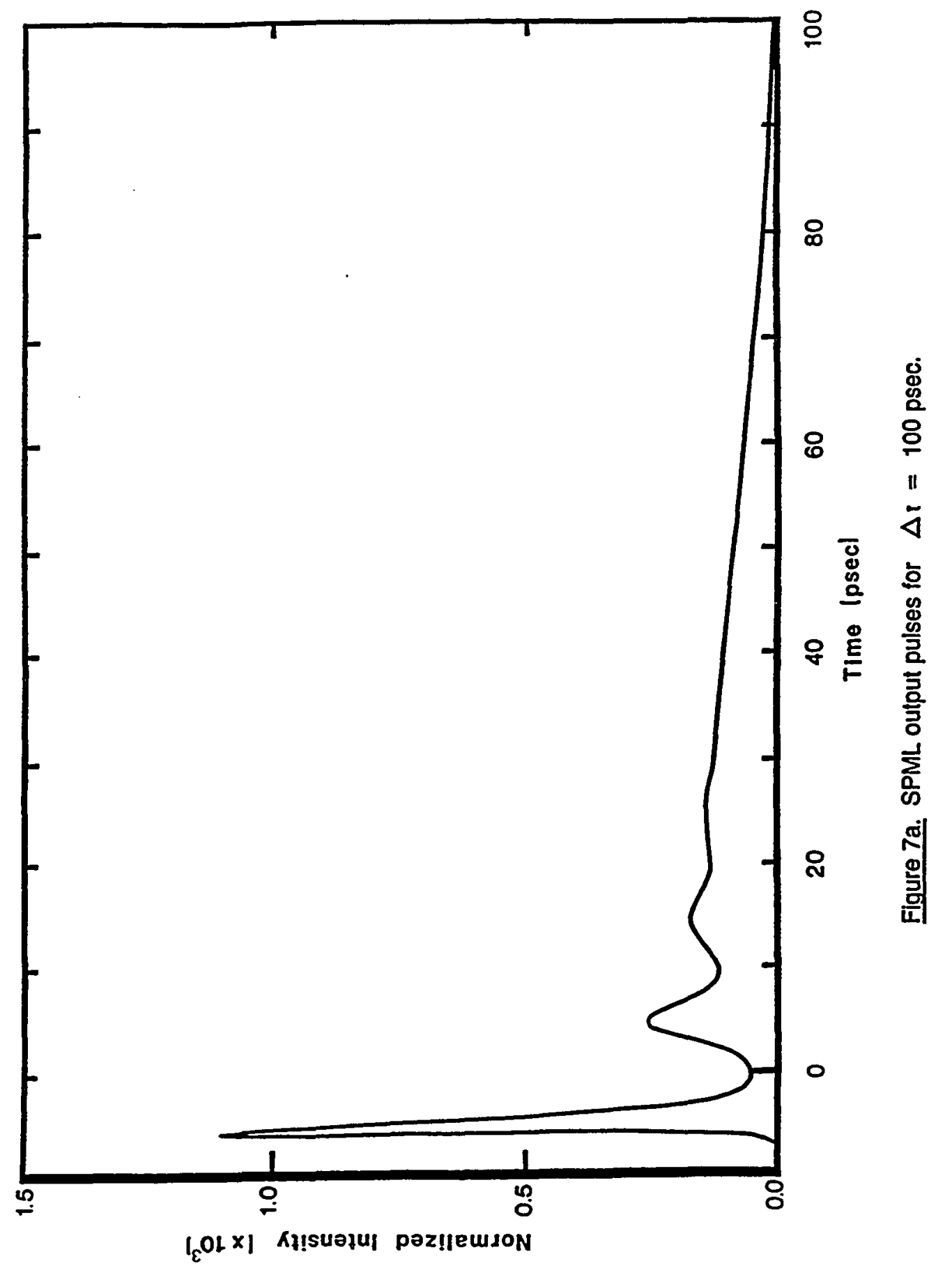




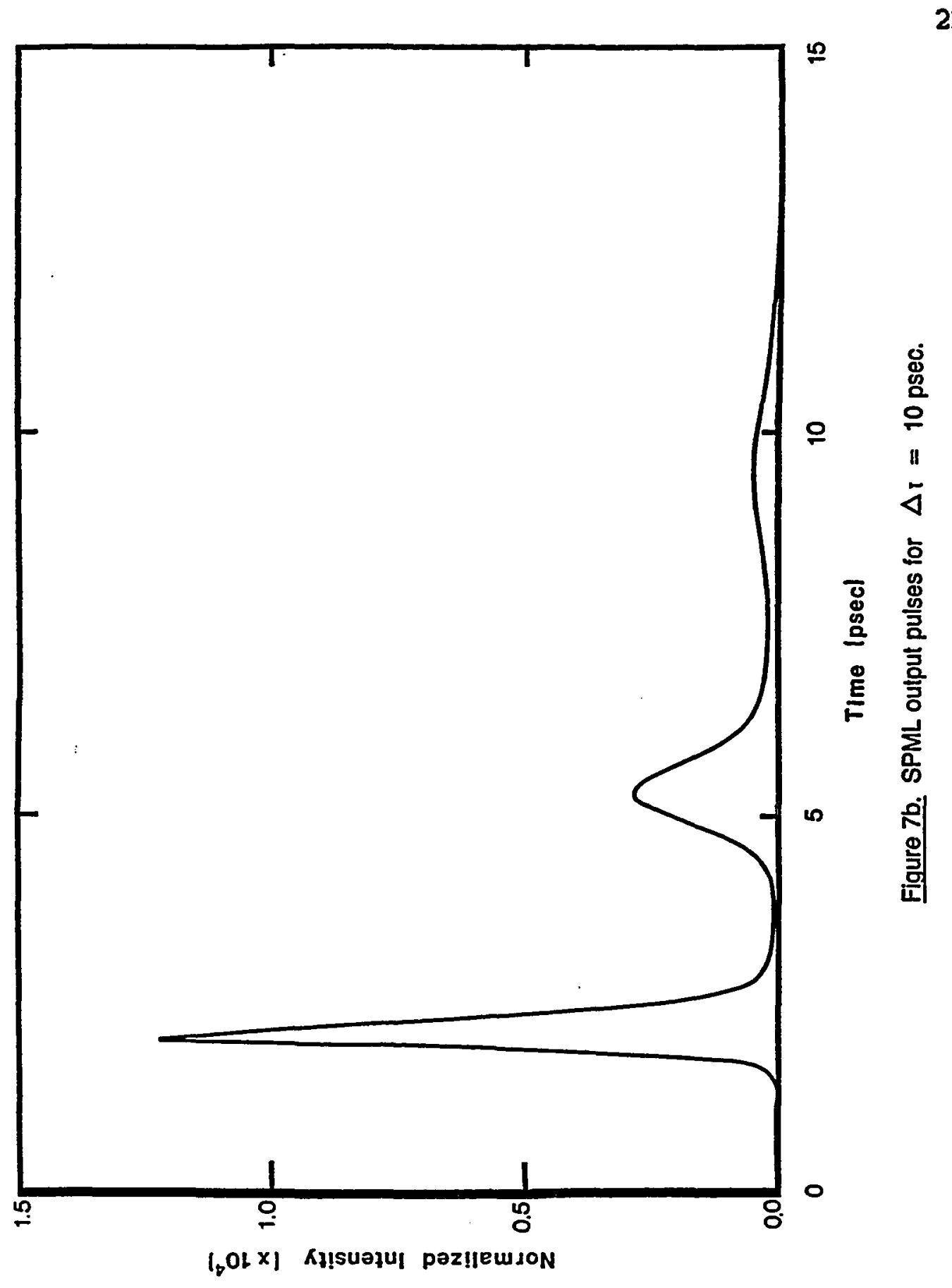




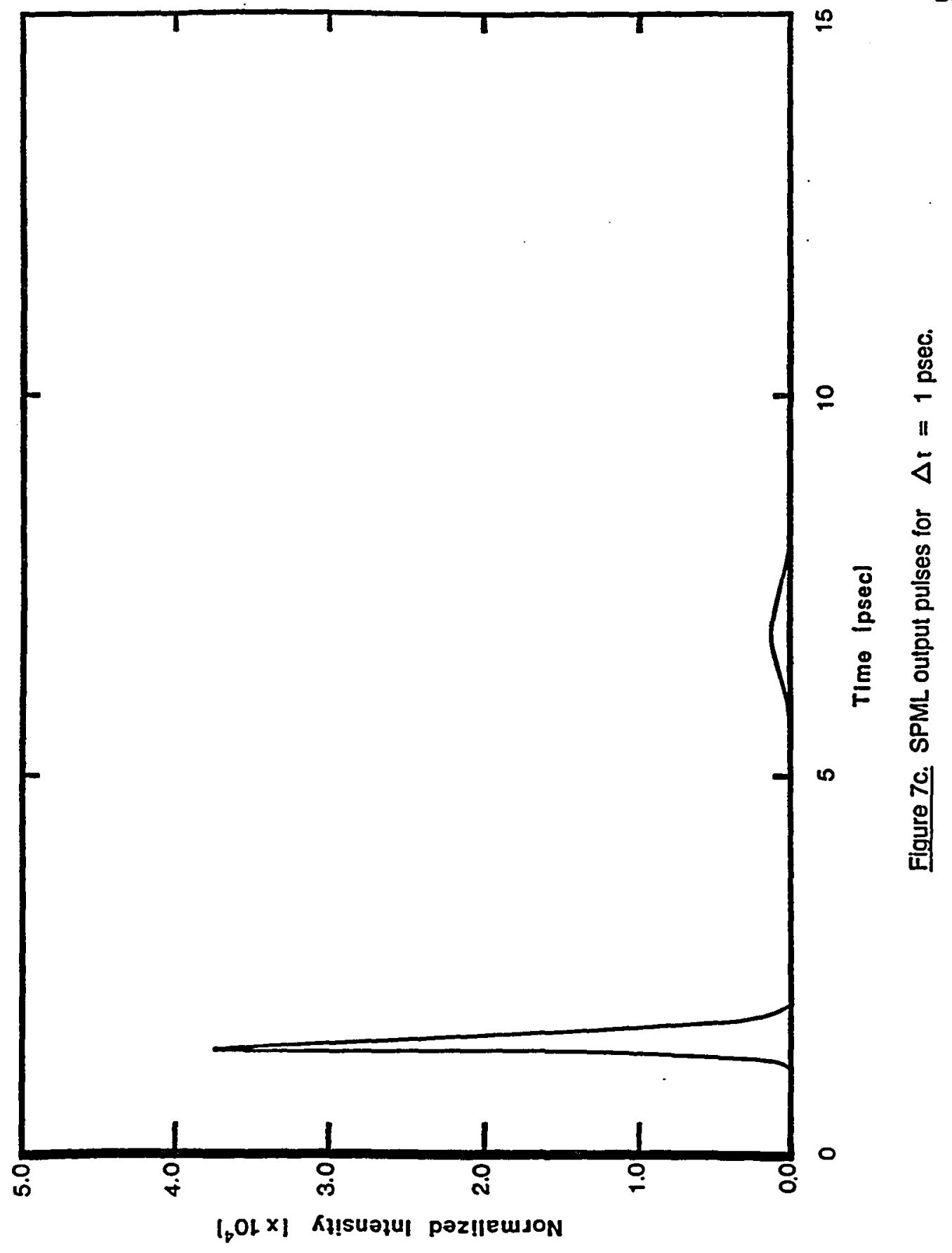


pulse for $\Delta \tau=3.5$ psec.

Our results so far have been limited to a symmetric Gaussian pump pulse. If one allows for asymmetric pulse shapes, then the rise time and the fall time become decoupled from the pulse width, and thus potentially become additional optimization parameters. To test, in particular, the cases of a short rise or fall time with a relatively long pulse time, we used a trapezoidal pump pulse of the form:

$$
P(t)=\begin{array}{ll}
0 & t \leq 0 \\
P_{0} t / \Delta \tau T_{1} & 0 \leq t \leq T_{1} \\
P_{0} / \Delta \tau & T_{1} \leq t \leq T_{2} \\
\left(P_{0} / \Delta \tau\right)\left(T_{3}-t\right) /\left(T_{3}-T_{2}\right) & T_{2} \leq t \leq T_{3} \\
0 & 0 \leq t
\end{array}
$$

for an extensive series of runs. Here $T_{1}$ is the total risetime, $\left(T_{3}-T_{2}\right)$ is the total fall time and $\Delta \tau=\left(T_{3}+T_{2}-T_{1}\right) / 2$ is the FWHM pulse length.

After checking that the discontinuous nature of this simple shape did not significantly effect the output, a wide range of runs were done with different risetimes and fall times. Though it might be plausible to expect that for a given $\Delta t$, a shorter risetime would result in a shorter output pulse, we found no trend to support this. Further, in some instances, a shorter risetime resulted in a longer $t_{p}$, a result which in some cases implies that the complicated switching dynamics of a SPML laser depends as much on when the pump pulse turns off as on how fast the energy is delivered. Also of importance is the integrating nature of the gain medium which comes from its finite response time. Simply put, there are regimes 
of operation where the integral of the pump is more important to the output than the form of the pump itself. Consequently, it might only be in rare instances where engineered pump pulse shaping becomes important, particularly if this comes at the expense of pump level and overall pump pulse width.

\section{COHERENCE EFFECTS}

An important distinction of the model used here and derived in [24] is its inclusion of semiclassical effects, or more specifically in its consideration of a finite coherence time, $T_{S}=50 \mathrm{fsec}$. At this value, it is reasonable to expect coherence effects to become more pronounced as the system dynamics become faster, or in light of the above results, at high $r$ or small $\Delta \tau$. To test, in general, the importance of coherence effects, several representative runs were taken in the rate equation limit, $T_{S}=0$, for various $\Delta \tau$ 's. The pertinent equations come from the adiabatic elimination of the polarization, Q, from (1) - (4) and are:

$$
\begin{aligned}
& \mathrm{dD} / \mathrm{dt}=-1 / \tau_{2}\left[\left(1+\tau_{2} / 2 \tau_{1}\right) D+\left(1-\tau_{2} / 2 \tau_{1}\right) M+2 A^{2} D x^{2}-P x^{2}\right] \\
& d M / d t=-1 / \tau_{2}\left[-\left(\tau_{2} / 2 \tau_{1}\right) D+\left(\tau_{2} / 2 \tau_{1}\right) M-P x^{2}\right] \\
& d A / d t=-L / 2 t_{C} \Delta L\left[A-\int_{0}^{1} A D x^{2} d x\right]
\end{aligned}
$$

It was shown in [24] that coherence effects manifest themselves most significantly in the behavior of the satellite pulses for small detunings. Physically, a finite coherence time implies that the 
polarization depends on past states of the field and therefore in the SPML laser case we are, when we include semiclassical effects, carefully modeling the evolution of a trailing satellite pulse as it is acted upon by a molecular ensemble dressed by an intense primary pulse. However we saw above that near the delta function limit, no satellite pulses are formed, and so semiclassical effects in SPML lasers, in one sense, become less important as $\Delta \tau$ is decreased.

A finite coherence time also limits how fast the gain can react and consequently semiclassical effects also manifest themselves measurably in the character of the primary pulse at all $\Delta \tau$ 's considered. Because of this additional lag, the pulse width, $t_{p}$, calculated using semiclassical effects is always longer than those calculated in the rate equation limit. For longer $\Delta \tau$ 's, the calculated peak intensity is higher when semiclassical effects are included but for shorter $\Delta \tau$ 's, the opposite is true. As shown in Fig. 8, the rate equation error in these two quantities, although only about 208 on average, is not a simple function of $\Delta \tau$. The additional delay induced by a nonzero $T_{S}$ also implies that $T_{\text {pk }}$ occurs much later in a semiclassical model than in the rate equation picture. For example, at $\mathrm{r}=2.0$, and $\Delta \mathrm{L}=10$ microns, $\mathrm{T}_{\mathrm{pk}}$ in the delta function limit is 1.5 psec for the rate equation calculations and $2.5 \mathrm{psec}$ for the semiclassical theory.

\section{CONCLUSIONS}

In this chapter we have studied the role of the pump pulse in the output of a SPML rhodamine 6G dye laser. In particular we focused on two key parameters associated with a Gaussian pump - 
its width, $\Delta \tau$, and its level with respect to threshold, $r$. Our results for $t_{p}$, the output pulse width, can be summarized in the contour plot of Fig. 9, where lines of constant $t_{p}$ are plotted against $r$ and $\Delta \tau$. As $\mathrm{r}$ increases and $\Delta \tau$ decreases, $t_{\mathrm{p}}$ gets shorter. The increase in spacing between adjacent curves in the upper right hand corner represents a case of diminishing returns. For the rhodamine $6 \mathbf{G}$ laser, state of the art technology is already near this regime, and further shortening of pulses from rhodamine lasers will most likely come from additional system elements such as intracavity saturable absorbers or external cavity fiber/grating compressors. On the other hand, we believe that the trends predicted in this work are general enough to be representative of most synchronously pumped, mode-locked systems. Consequently one might expect improvements from SPML lasers operating at different wavelengths as their pump sources mature. 


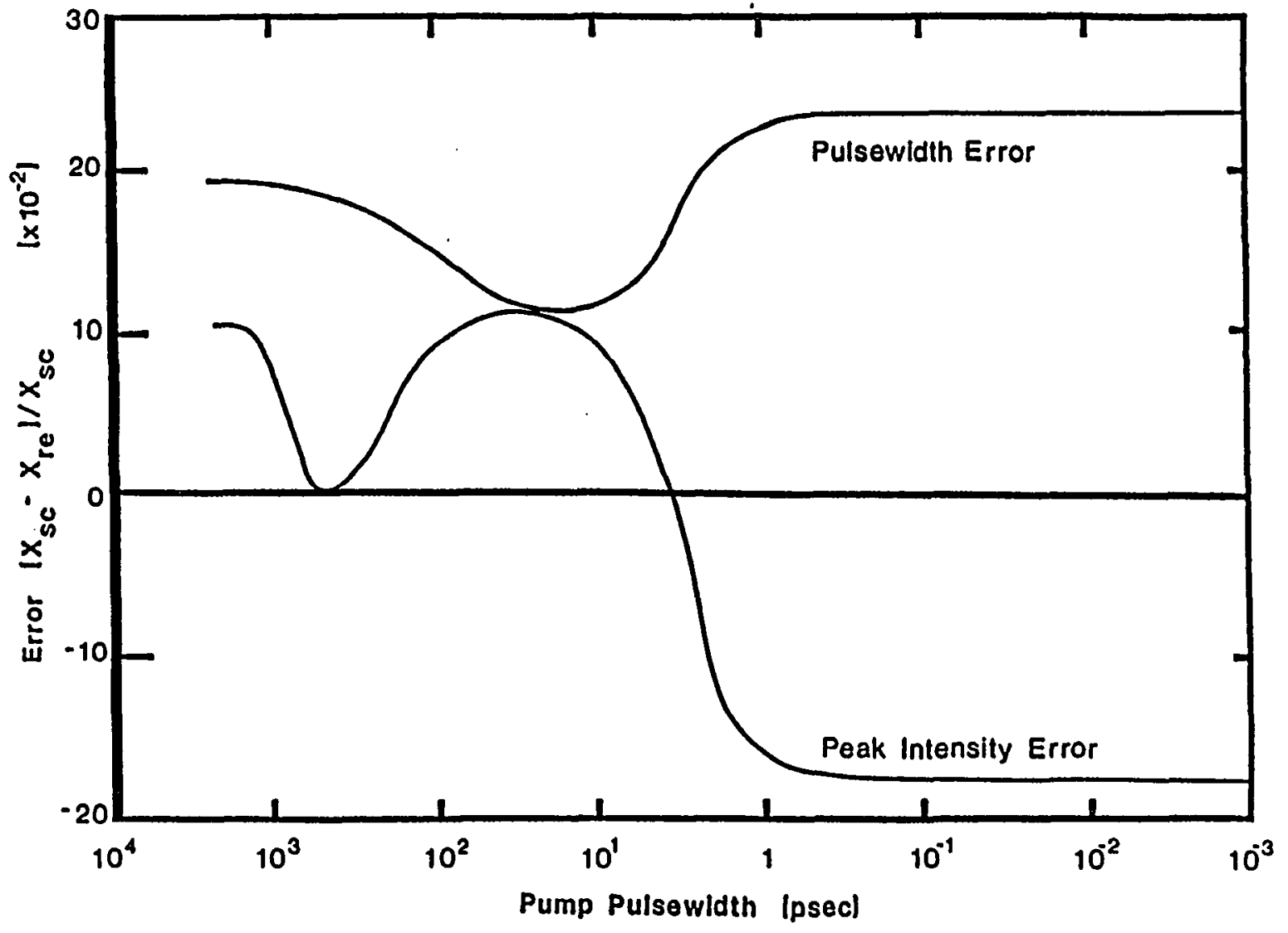

Figure 8. Error due to the rate equation approximation. The error In the pulse width and the intensity that occurs in the rate equation limit plotted as a function of the pump pulse width. The error is defined here as the difference between the semiclassical value and the rate equation value, normalized to the semiclassical value. 


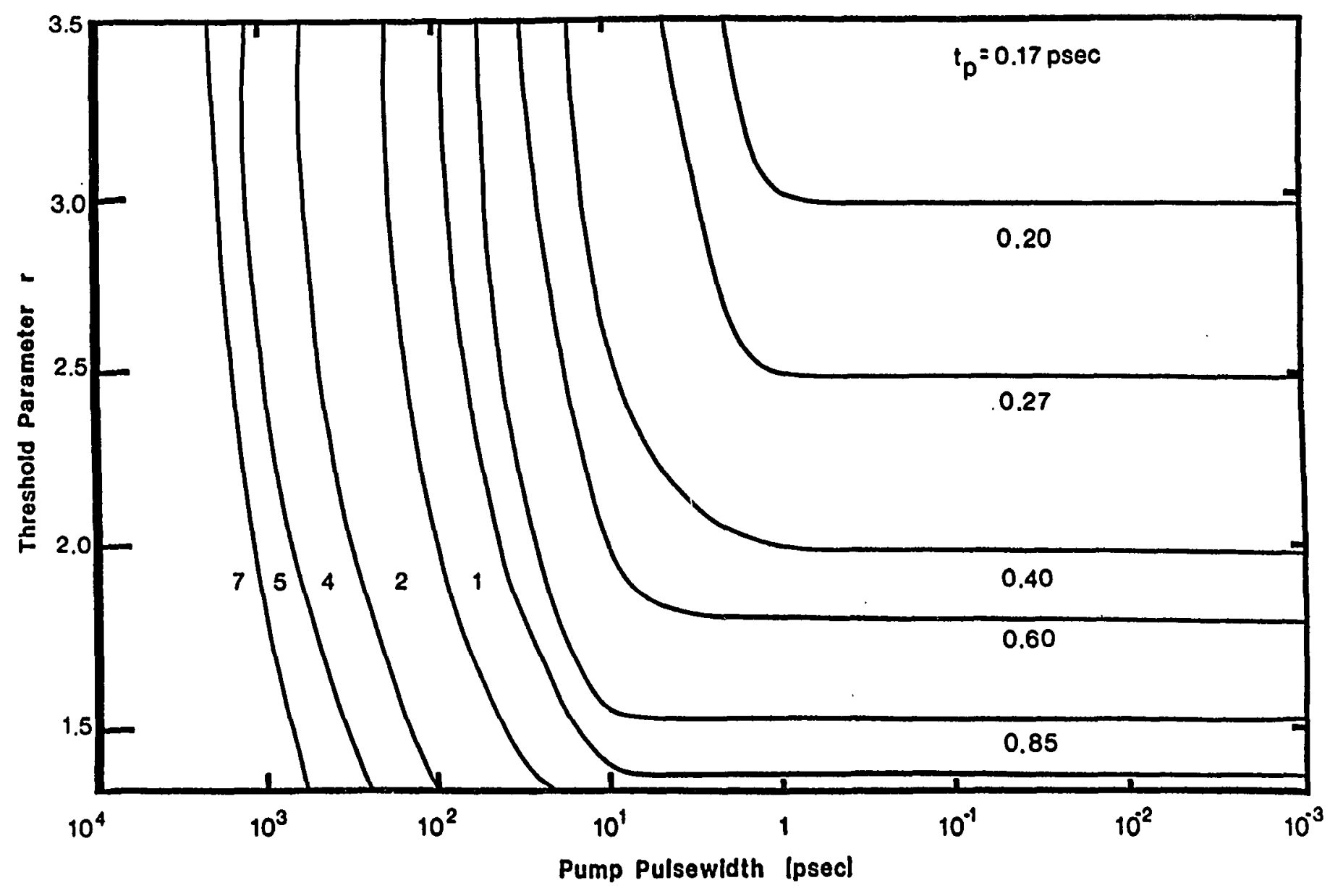

Flgure 9. SPML pulse width contours in $r$ and $\Delta t$ space. 


\section{CHAPTER III}

THEORY OF A SYNCHRONOUSLY PUMPED MODE-LOCKED DYE LASER IN THE SHORT PUMP PULSE LIMIT

\section{INTRODUCTION}

The analysis of pump pulse effects in synchronously pumped mode-locked (SPML) dye lasers in the last chapter revealed that several of the most advanced SPML dye lasers $[7,8]$ operate in the regime where the character of the output pulse is unresponsive to any further shortening of the pump pulse. Experimentally, this situation occurs when the pump pulse of a SPML rhodamine dye laser system is shorter than a few psec, a limit that has recently been achieved with fiber/grating compressed, frequency doubled Nd:YAG lasers [7,8]. This study would also apply to SPML dye laser pumped SPML dye lasers [26]. Mathematically, this situation corresponds to a Dirac delta function pump and therefore leads to a great simplification of the underlying model. In effect, the forced system becomes unforced, and the SPML dye laser becomes prepumped every cavity roundtrip.

In this chapter we reduce a detailed, proven, nonlinear dynamical model $[24,27]$ to a point where analytic exploration becomes possible, while the character of SPML operation is retained. In particular, we consider two limits, both of which predict pulses that are in qualitative agreement with the full model that agrees quantitatively with experiment. The first limit considered is the rate equation approximation, and here we find a 
parallel between SPML systems and giant pulsed, or Q-switched lasers [28-31]. That gain switching should follow as an extreme form of gain modulation seems reasonable - even intuitive, however the traditional Q-Switching model neglects semiclassical effects which we find to be equally important to pulse development on the time scales considered here. Our second limit is one of zero length mismatch between the pump laser and the SPML laser and mathematically corresponds to what is now called the bad cavity limit. The resulting set of coherent equations is somewhat simpler than the rate equations, but also yields short pulse solutions, and these solutions may be expressed analytically. Any further simplification of either of these limits causes the pulsed behavior to disappear.

In the next section we review the model derived in reference [24] and studied in the last chapter. We consider the delta function pump pulse limit and several other simplifying assumptions. The rate equation limit is presented in section 3 , and parallels to Q-switched operation are discussed. In particular, we apply the approximate analytic solutions for Q-switched laser pulses developed in reference [32] to SPML dye laser operation. We also derive approximate analytic formulas for the peak intensity and the pulse width. Section 4 considers the zero detuning limit and demonstrates the importance of coherence effects in SPML operation in the short pump pulse limit. In our conclusion we use our findings to comment on the character of SPML operation and the practical significance of an ultrashort pump pulse. 
REVIEW AND INITIAL SIMPLIFICATIONS OF THE MODEL

Our starting point is the semiclassical dye laser amplifier model developed in reference [24] and studied in the last chapter. Begining with the density matrix equations and Maxwell's equations, reference [24] developed a set of ordinary, nonlinear differential equations that govern the interplay of the electric field with the molecular populations and the polarization. When the electric field is normalized to its steady state gain saturating value, this set of equations may be written as:

$$
\begin{aligned}
& \mathrm{dD} / \mathrm{dt}=-\left(1 / \tau_{2}\right)\left[\left(1+\tau_{2} / 2 \tau_{1}\right) D+\left(1-\tau_{2} / 2 \tau_{1}\right) M+2 Q A x-P x^{2}\right] \\
& d M / d t=-\left(1 / \tau_{2}\right)\left[-\left(\tau_{2} / 2 \tau_{1}\right) D+\left(\tau_{2} / 2 \tau_{1}\right) M-P x^{2}\right] \\
& d Q / d t=-\left(1 / T_{S}\right)[Q-A D x] \\
& d A / d t=-\left(L / 2 t_{c} \Delta L\right)\left[A-\int_{0}^{1} Q x d x\right]
\end{aligned}
$$

In equations (11) - (14), D is a normalized population difference, $M$ is a normalized population sum, $Q$ is a normalized polarization and $A$ is a normalized electric field. The dye decay time from the $S_{1}$ band to the $S_{0}$ band is $\tau_{2}$, and $\tau_{1}$ is the lower manifold intraband vibrational relaxation time. $T_{S}$ is the semiclassical coherence time. A convenient benchmark system is the rhodamine $6 G$ laser for which one finds the approximate values, $\tau_{1}=1 \mathrm{psec}, \tau_{2}$ $=5 \mathrm{nsec}$, and $T_{S}=50 \mathrm{fsec}$. The dynamical rate constant associated with the field is 
composed of the cavity length, $\mathrm{L}$, the cavity lifetime, $t_{C}$, and the optical length mismatch, $\Delta L$, which is the amount by which the dye laser cavity length exceeds that of the pump laser. Typically, $\mathrm{L}=1.8 \mathrm{~m}, \mathrm{t}_{\mathrm{c}}=10 \mathrm{nsec}$, and $\Delta \mathrm{L}$ is small, approximately $0-30$ microns. For the shortest output pulses, $\Delta \mathrm{L}$ is a key experimental adjustment. This model is also general enough to explicitly include the orientation of the molecular dipoles, $x=\cos (\theta)$. Thus, in general, equations (11) - (13) refer specifically to only one orientational class and the individual classes contribute to the field through the integral over $x$ in equation (14).

$P(t)$ is the normalized pump function and its effects were explored in detail in the last chapter for the Gaussian shape:

$P(t)=P_{0}\left(2 / \Delta_{\tau}\right)(\ln 2 / \pi)^{1 / 2} \exp \left[-\left(2 t / \Delta_{\tau}\right)^{2} \ln 2\right]$

In equation (15), $\Delta \mathrm{t}$ is the full-width at half-maximum (FWHM) pulse width and $P_{0}$ is a measure of the pump level, which in most practical cases is directly proportional to the threshold parameter, $r$.

of specific interest in the last chapter was the change in the SPML output pulse as the pump pulse width was decreased. Figure 10 shows the decrease in the output pulse width as $\Delta \tau$ is shortened through several orders of magnitude. Like all aspects of the SPML output pulse character, there is no change in the SPML pulse width as $\Delta \tau$ is shortened beyond a few psec. This point is central to this paper because it shows that for $\Delta \tau \leq 1 \mathrm{psec}, \mathrm{P}(\mathrm{t})$ may be replaced with a Dirac delta function, $P(t)=P_{0} \delta(t)$. $A$ typical pulse predicted by set (11) - (15) in the delta function limit 


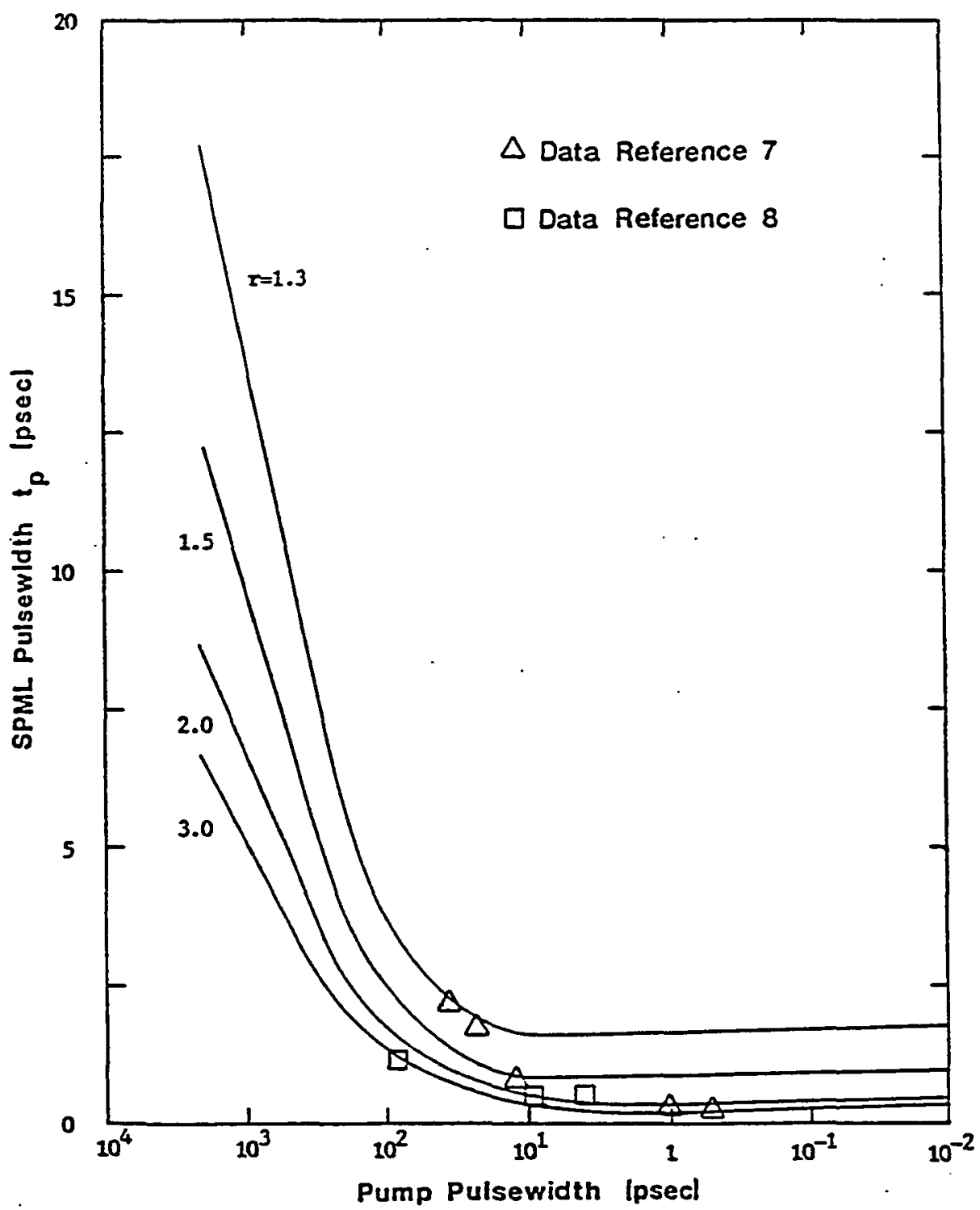

Eigure 10. SPML pulse width versus pump pulse width. 
is shown in Fig. 11. In the remainder of this section we simplify the set (11) - (15) using various approximations in order to study the nature of SPML dye lasers in the delta function limit.

One of the biggest complications to the set (11) - (14) is the dipole orientation integral in the field equation. While orientational effects have been shown to be of importance in quantitative modeling of the output pulse shape, dipole orientation is not essential to the general nature of SPML operation [24]. More formally, one might argue that with parallel pump and signal polarizations and slow rotational relaxation, only one orientational class of dye molecules would contribute to the field. Hence we consider here a unidirectional orientational distribution $[24,33]$ whereby the set (11) - (15) reduces to:

$$
\begin{aligned}
& d D / d t=-\left(1 / \tau_{2}\right)\left[\left(1+\tau_{2} / 2 \tau_{1}\right) D+\left(1-\tau_{2} / 2 \tau_{1}\right) M+2 Q A-P_{0} \delta(t)\right] \\
& d M / d t=-\left(1 / \tau_{2}\right)\left[-\left(\tau_{2} / 2 \tau_{1}\right) D+\left(\tau_{2} / 2 \tau_{1}\right) M-P_{0} \delta(t)\right] \\
& d Q / d t=-\left(1 / T_{S}\right)[Q-A D] \\
& d A / d t=-\left(L / 2 t_{C} \Delta L\right)[A-Q]
\end{aligned}
$$

In equations (16) - (19) we have also written the pump function explicitly as a delta function.

For values of $\tau_{1}$ and $\tau_{2}$ relevant to typical dye lasers, we note that $\tau_{2} / 2 \tau_{1} \gg 1$, and to good approximation, equation (16) may be rewritten as: 


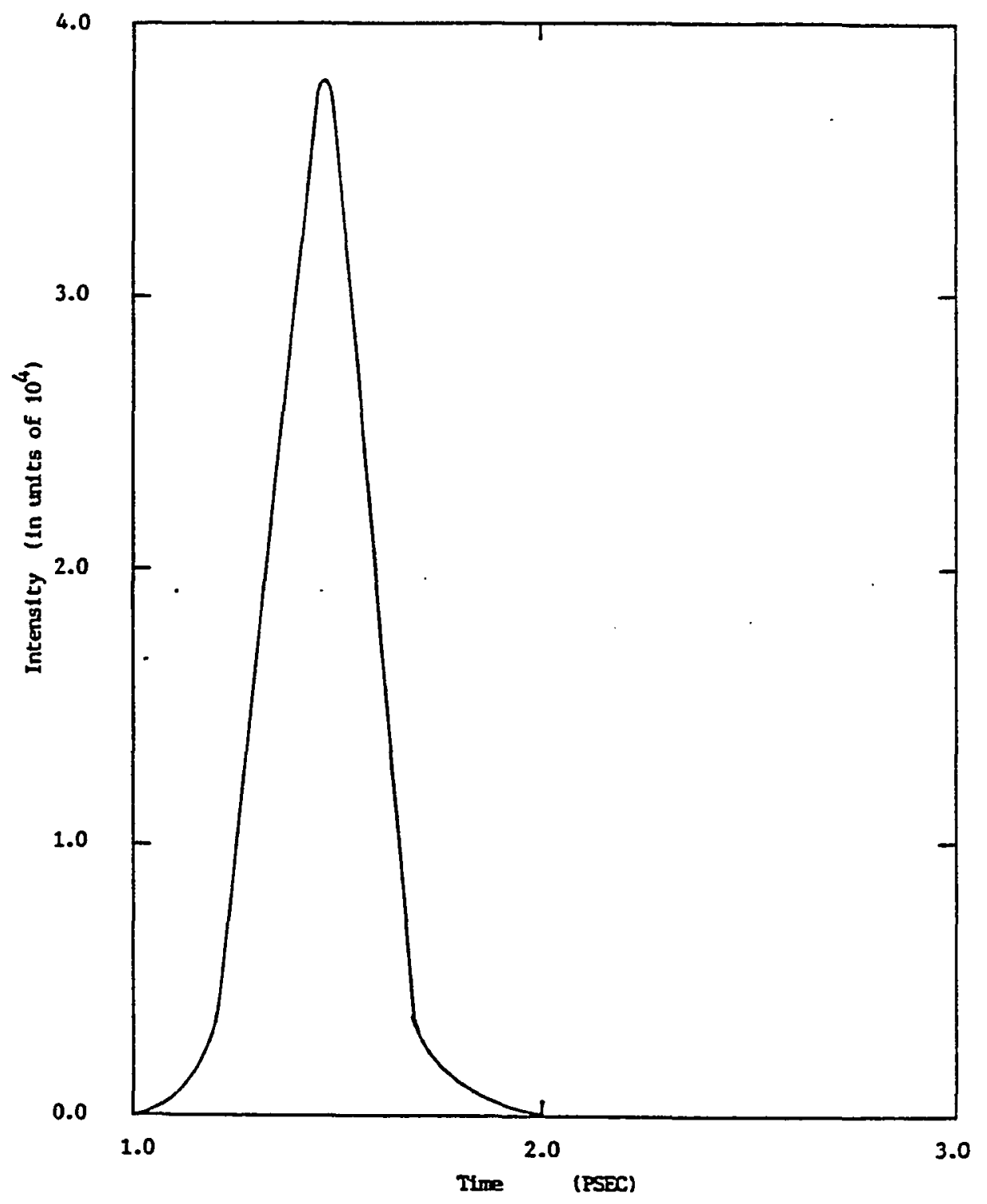

Eisure Il SPML output pulse. 
$d D / d t=-\left(1 / \tau_{2}\right)\left[\left(\tau_{2} / 2 \tau_{1}\right) D-\left(\tau^{2} / 2 \tau_{1}\right) M+2 Q A-P_{o} \delta(t)\right]$

Considering the femtosecond output pulses found to be produced by this system in the delta function pumping limit, it seems reasonable to neglect the nonradiative relaxation from the lower laser state. This limit of $\tau_{1}$ long compared to the lasing action allows us to combine equations (17) and (20), and to eliminate the population sum, $M$, and its dynamical equation. The population difference equation then becomes:

$d D / d t=-\left(1 / \tau_{2}\right)\left[2 Q A-P_{0} 8(t)\right]$

The delta function in (21) is only nonzero at $t=0$ and therefore serves to set the initial conditions appropriate to the pumping level. At $t=0, A \approx 0$ and equation (21) is decoupled from equations (18) and (19). We integrate from $t=0^{-}$to $t=0^{+}$, through the delta function, to establish the initial condition for $D$ at a time $0^{+}$. Thus, immediately after the pump function, $D\left(\mathrm{O}^{+}\right)=\mathrm{P}_{\mathrm{O}} / \tau_{2}=r$, the threshold parameter. The corresponding initial condition for the field should be a value that represents an approximate level of spontaneous emission. For our work, this $A_{0}$ is chosen to be $10^{-10}$. For all times greater than 0 , then, our set of equations is:

$$
\begin{aligned}
& \mathrm{dD} / \mathrm{dt}=-\left(1 / \tau_{2}\right)[2 \mathrm{QA}] \\
& \mathrm{dQ} / \mathrm{dt}=-\left(1 / T_{S}\right)[\mathrm{Q}-\mathrm{AD}]
\end{aligned}
$$


$\mathrm{dA} / \mathrm{dt}=-\left(\mathrm{L} / 2 \mathrm{t}_{c} \Delta \mathrm{L}\right)[\mathrm{A}-\mathrm{Q}]$

The output pulse predicted by this set of equations is shown in Fig. 12. Neglecting orientational effects and simplifying the energy level scheme causes the output pulse to form 4 picoseconds later. Notably, the peak intensity is essentially unchanged with these approximations, and there is only a slight shortening in the pulse width. We also note that equations (22), (23) and (24) correspond to the much considered Lorenz equations [34 - 36] for the case of zero forcing.

In the following two sections of this paper, we consider equations (22), (23) and (24) in two limits: the rate equation limit, $T_{S}=0$, and the zero detuning limit, $\Delta L=0$. Interestingly, both limits yield pulses that are at least qualitatively similar to those predicted by the initial set (11) - (15).

\section{RATE EQUATION LIMIT}

In the rate equation approximation, one ignores coherence effects and therefore the dependence of the polarization on past values of the electric field. Since a finite coherence time represents a tempoaral lag between the electric field and the polarization, coherence effects play an important role in limiting the magnitude and width of short optical pulses $[24,27,37]$. However, the error involved in the rate equation limit is typically less than a factor of two for these systems $[24,27]$ and thus, for a qualitative study, 


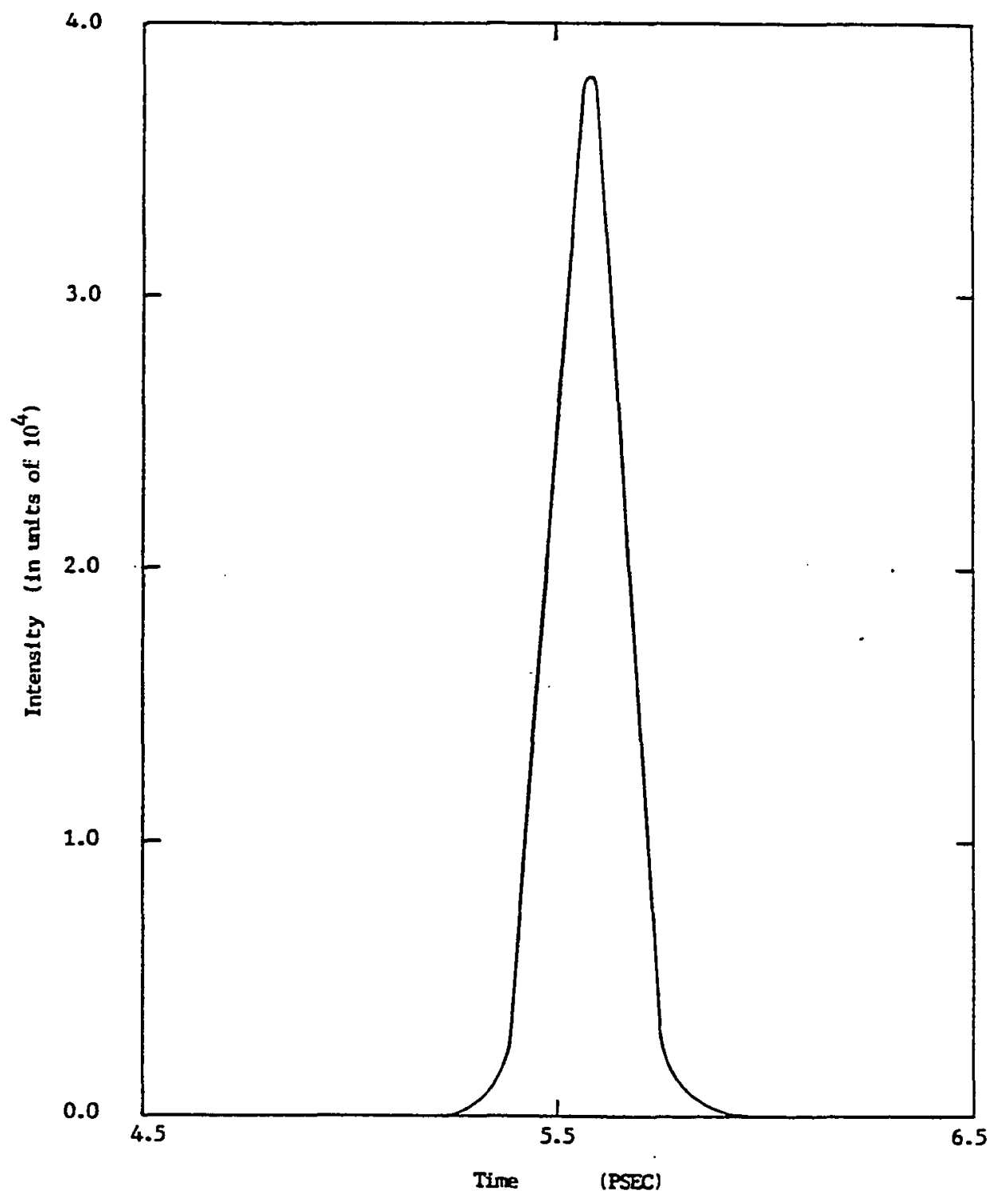

Elgure 12, SPML output pulse. 
the rate equation approximation is the limit of $T_{S} \approx 0$. From equation (23), then, $Q=A D$, and our set may be written in terms of the normalized population difference, $D$, and the intensity, $I \equiv$ $A^{2}$.

$\mathrm{dD} / \mathrm{dt}=-\left(1 / \tau_{2}\right)[2 \mathrm{ID}]$

$\mathrm{dI} / \mathrm{dt}=-\left(\mathrm{L} / \mathrm{t}_{\mathrm{c}} \Delta \mathrm{L}\right)[\mathrm{I}-\mathrm{ID}]$

Equations (25) and (26) are exactly the form of the equations that describe giant pulses from Q-switched lasers [29,30,31] and it is useful to reinterpret the results of these earlier studies in the context of mode-locked lasers.

Dividing (25) by (26) and integrating, yields the intensity as a function of D. Noting from equation (26) that the peak intensity occurs at $D=1$, we may recast the familiar formula for the peak intensity of a Q-switched laser pulse in terms of SPML laser parameters.

$\mathrm{I}_{\mathrm{pk}}=\tau_{2} \mathrm{~L} / 2 \mathrm{t}_{c} \Delta \mathrm{L}\left[\mathrm{r}-\ln r-1+2 \mathrm{t}_{c} \Delta \mathrm{LI} \mathrm{I}_{\mathrm{o}} \tau_{2} \mathrm{~L}\right]$

Figure 13 includes a plot of equation (27) as a function of $r$ and compares it to the peak intensity calculated by equations (11) - (15). We see that over the range of interest, the agreement is of the correct order of magnitude.

We know of no complete analytic solution to the set of equations (25) and (26). A typical approach in analytic studies involves fitting the numerical solution of (25) and (26) to a family 


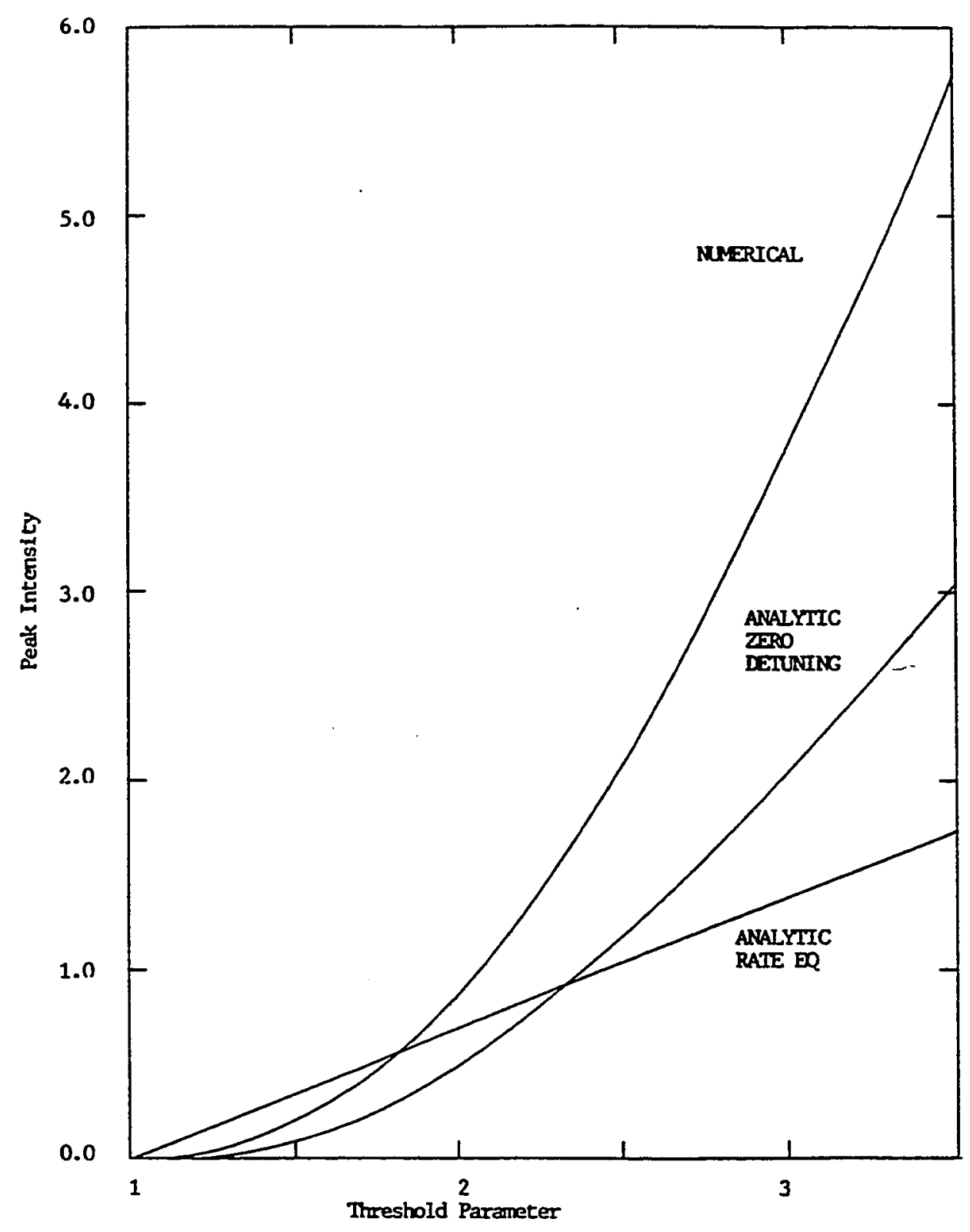

Figure 13. SPML peak intensity versus the threshold parameter. The intensity scale is in units of $10^{4}$ for the numerical and the rate equation curves, but it is in units of $10^{5}$ for the zero detuning curve. 
of analytic pulse shapes [32]. Before considering specific examples of such pulses and their autocorrelations, we will study (25) and (26) in two limits that yield, respectively, the leading edge and the falling edge of the pulse. These limits will suggest an appropriate pulse form and will allow us to estimate the mode-locked pulse width analytically.

The leading edge of the pulse is essentially governed by a maximal, constant population difference, $D \approx r$. This assumption linearizes equation (26) and allows direct solution of the set. The resulting intensity is a rising exponential:

$I(t)=I_{0} \exp \left[L(r-1) t / t_{c} \Delta L\right]$

The falling edge of the pulse may be characterized by a depleted gain, $D=0$. With this approximation, equation (26) again becomes decoupled from (25) and may be solved to yield an exponentially decaying intensity:

$I(t) \propto \exp \left[-L t / t_{c} \Delta L\right]$

One can crudely approximate the pulse width, $t_{p}$, by the sum of the rise time in (28) and the fall time in (29):

$$
t_{p}=\left(r t_{c} \Delta L\right) /[L(r-1)]
$$

This equation represents a lower limit on $t_{p}$. Figure 14 compares the $r$ dependence of $t_{p}$ as predicted by equation (30) to the FWHM 


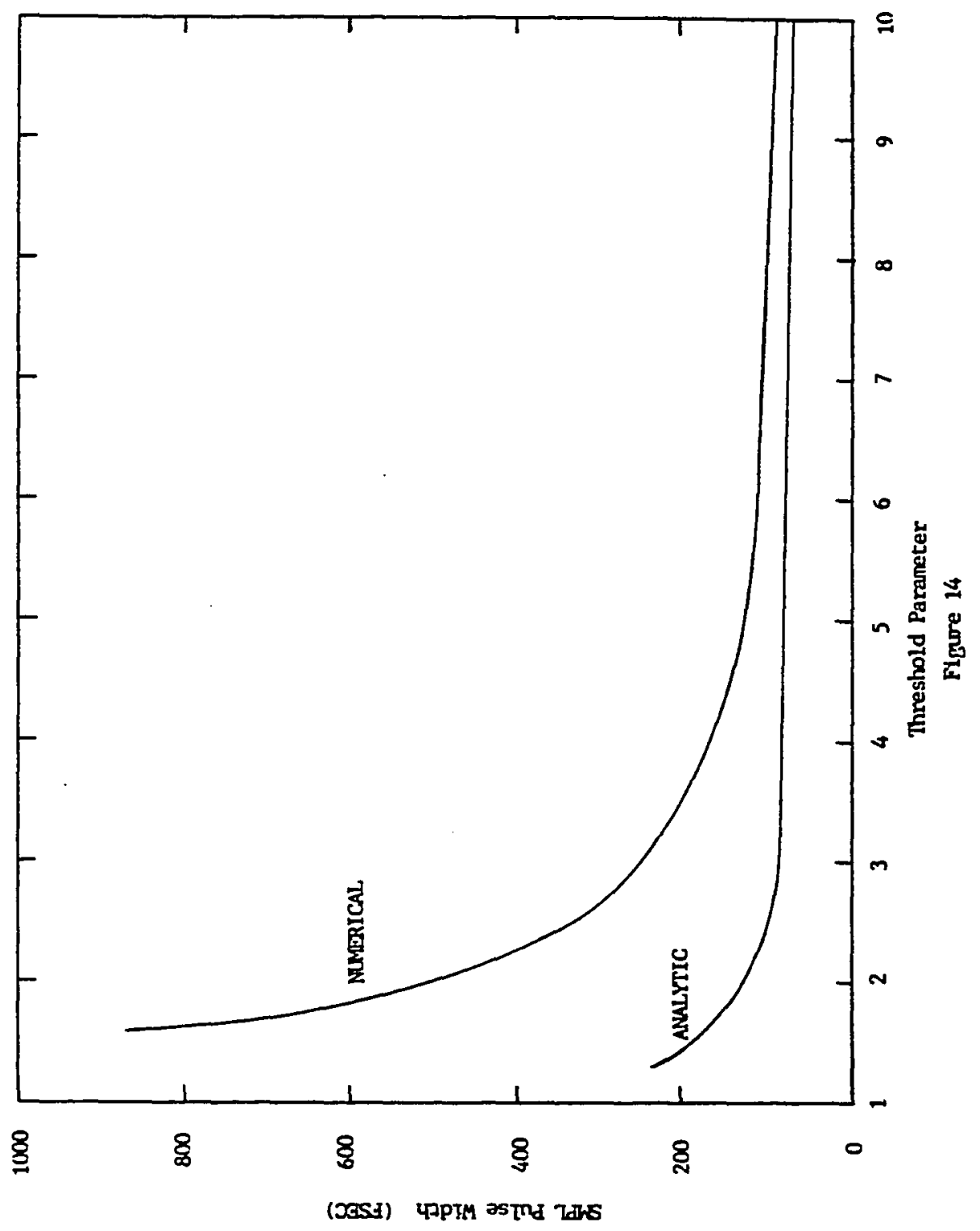

Figure 14. SPML puise width versus the threshold parameter. 
$t_{p}$ given by equations (11) $-(15)$ for $\Delta L=10$ microns and $t_{c}=10$ nsec. In the large $r$ limit we see a reasonable, order of magnitude agreement between the two. This agreement, however, deteriorates quickly as $r$ decreases and the consequences of our approximations become evident.

In selecting an analytic pulse shape, one might consider numerically approximating equations (25) and (26), by a curve fitting technique, to the family of symmetric pulses,

$I(t)=I_{p}\left\{\operatorname{sech}\left[r L\left(t-t_{p}\right) /\left(4 n t_{c} \Delta L\right)\right]\right\}^{n}$

or the family of asymmetric pulses,

$I(t)=I_{p}\left\{1+\left(t-t_{p}\right) / n t_{0}\right\}^{n} \exp \left[-\left(t-t_{p}\right) / t_{0}\right]$

for values of $t>t_{p}-n t_{0}$. A particular advantage of the pulses of the form of (32) is that one can calculate analytically, their autocorrelation, which is the experimentally measured parameter. For example, the autocorrelation of (32) for $n=1$ is

$$
(|\tau|+1) \exp (2-|\tau|)
$$

and for $n=2$ is

$$
\left(\tau^{2}+3|\tau|+3\right) \exp (4-|\tau|)
$$

The normalized time, $\tau$, in (33) and (34) is in units of $t_{0}$. In light of equations (28) and (29), however, one desires a pulse shape with 
an exponential rise and fall, and an example that satisfies these conditions is [32]:

$I(t)=I_{p k} r \exp \left[-L\left(t-T_{p k}\right) /\left(t_{c} \Delta L\right)\right]\left\{\exp \left[-r L\left(t-T_{p k}\right) /\left(t_{c} \Delta L\right)\right]+r-1\right\}^{-1}$

Here, $\mathrm{T}_{\mathrm{pk}}$ is the time at which the peak of the pulse occurs and may be calculated approximately from the initial (spontaneous emission) intensity, $I_{0}$, by,

$T_{p k}=\left(t_{c} \Delta L / L(1-r)\right)\left[\ln \left(2 I_{o} t_{c} \Delta L(r-\ln r-1) / \tau_{2} L r\right)\right]$

The numerical solution to the set (25) and (26) is shown in Fig. 15a and equation (36) is shown in Fig. 15b.

\section{ZERO DETUNING LIMIT}

One might also consider equations (22) - (24) in the limit of very small cavity detunings. If $\Delta \mathrm{L}$ is allowed to go to zero, equation (24) implies $A=Q$ and we may adiabatically eliminate the electric field, $A$. This approximation is mathematically identical to considering a very short cavity lifetime, $t_{c}$ and hence is sometimes refered to as the bad cavity limit in non-mode-locked lasers. This limit contrasts with the rate equation limit in that this approximation retains the semiclassical effects. With this approximation our governing set of equations becomes: 


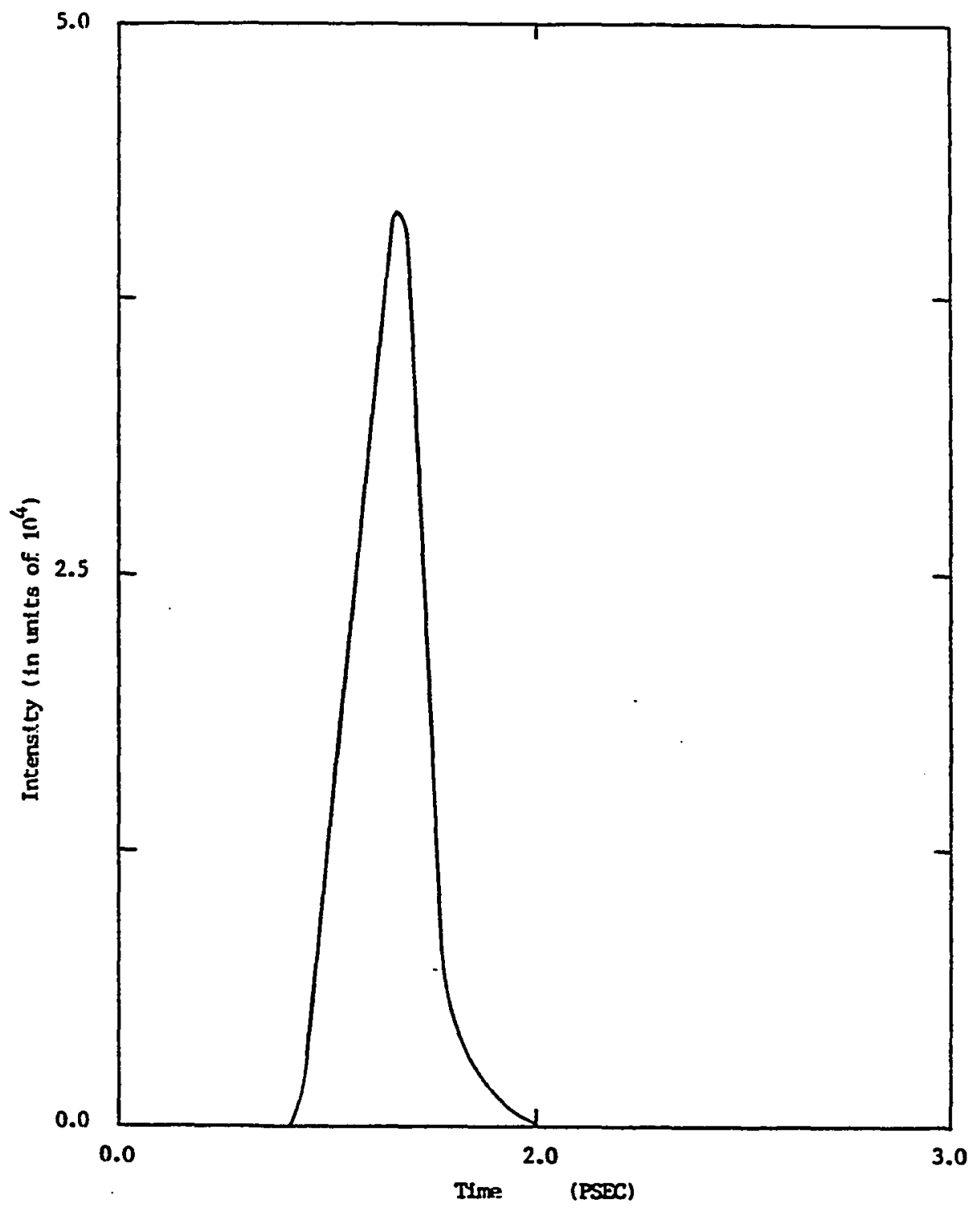

Eigure 15a. Analytic fit for the SPML output pulse in the rate equation limit. 


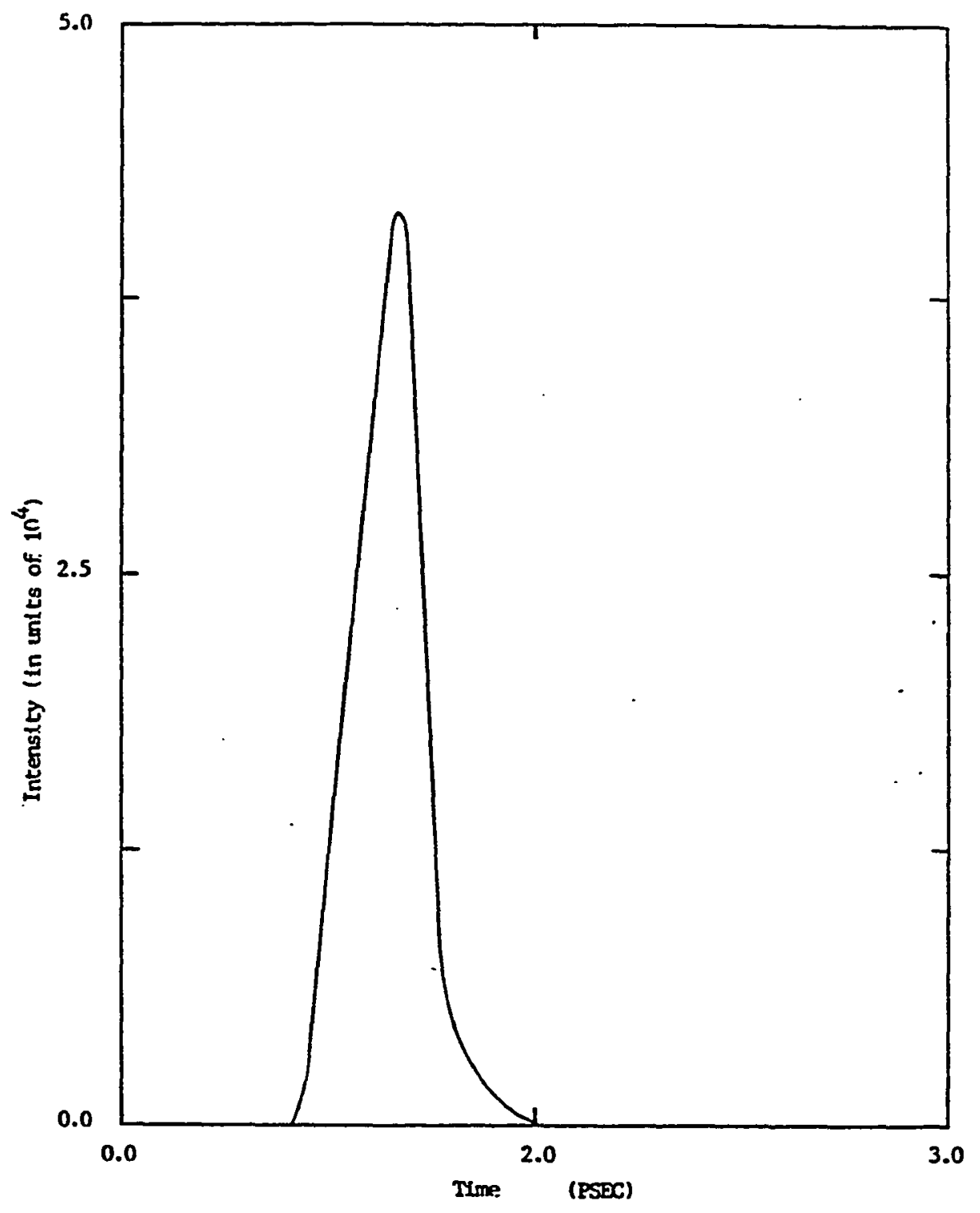

Figure 15b. Numerical SPML output pulse in the rate equation limit. 
$\mathrm{dD} / \mathrm{dt}=-\left(1 / \tau_{2}\right)[2 \mathrm{I}]$

$d I / d t=-\left(2 / T_{S}\right)[I-I D]$

We have identified the intensity, 1 , with the square of the polarization, $I=A^{2}=Q^{2}$. Noteworthy is that the population equation (37) is linear and hence is simpler than the population equation (25) of the rate equation limit. An analytic solution to (37) and (38) may be obtained subject to the initial conditions $\mathrm{D}\left(\mathrm{O}^{+}\right)$ $=\mathrm{r}, \mathrm{I}\left(\mathrm{O}^{+}\right)=\mathrm{I}_{\mathrm{O}}$, where $\mathrm{I}_{\mathrm{O}}$ represents the spontaneous emission. Dividing (37) by (38) and integrating gives the intensity as a function of the population difference.

$I(D)=\left(\tau_{2} / 2 T_{S}\right)\left[-D^{2}+2 D+r^{2}-2 r+2 T_{S} I_{0} / \tau_{2}\right]$

Noting that the peak intensity again occurs at $D=1$, allows us to write for $\mathrm{I}_{\mathrm{pk}}$ :

$\mathrm{I}_{\mathrm{pk}}=\left(\tau^{2} 2 / 2 \mathrm{~T}_{\mathrm{S}}\right)\left[(r-1)^{2}+2 \mathrm{~T}_{\mathrm{S}} \mathrm{J}_{\mathrm{O}} / \tau_{2}\right]$

Equation (40) is plotted in Fig. 13 as a function of $\mathrm{r}$ for a negligible value of $I_{0}$. We note that the quadratic form of equation $(40)$ is more faithful to the exact theory of equations (11) - (15) than its rate equation counterpart.

Substituting (39) into (37) gives a nonlinear differential equation for $D$ of the form: 
$\mathrm{dD} / \mathrm{dt}=\mathrm{aD} \mathrm{D}^{2}+\mathrm{bD}+\mathrm{c}$

where

$a=1 / T_{s}, \quad b=-2 / T_{S}$

and

$c=\left(-1 / T_{s}\right)\left[r^{2}-2 r+2 T_{s} I_{0} / \tau_{2}\right]$

Equation (41) is a constantly forced Ricatti equation that may be solved by making either the substitution:

$E(t)=[D(t)-a]^{-1}$

or the substitution:

$\left(T_{S / F(t)}\right) d F / d t=[1-D(t)]$

or by direct integration, which is the method that we will illustrate. Separating equation (41) yields the integral,

$\int_{r}^{D}\left[a D^{2}+b D+c\right]^{-1} d D=t$ 
This integral is well tabulated [38] and, pertinently, for $b^{2}, 4 a c$, the result is:

$t=\left(b^{2}-4 a c\right)^{1 / 2} \ln \frac{\left\{\left[2 a D+b-\left(b^{2}-4 a c\right)^{1 / 2}\right]\left[2 a r+b+\left(b^{2}-4 a c\right)^{1 / 2}\right]\right\}}{\left\{\left[2 a D+b+\left(b^{2}-4 a c\right)^{1 / 2}\right]\left[2 a r+b-\left(b^{2}-4 a c\right)^{1 / 2}\right]\right\}}$

Using equations (40), (42) and (46), we may solve for $D(t)$ explicitly:

$D(t)=$

$$
(1+k)-[r-1-k(r-k)][r-1+k]^{-1} \exp \left[\left(2 k / T_{S}\right) t\right]
$$

$$
1-[r-1-k][r-1+k]^{-1} \exp \left[\left(2 k / T_{S}\right) t\right]
$$

where

$k=\left(2 T_{S} I_{p k} / \tau_{2}\right)^{1 / 2}$

Substitution of (47) into (39) yields a closed expression for I(t) which is plotted in Fig. 16 for our typical values of $r=3, T_{S}=50$ fsec, $\tau_{2}=5$ nsec and the reasonable spontaneous emission level of $10^{-20}$. The solution has a pulsed character of reasonable shape and energy when compared to Fig. 11. However, the pulse width predicted by equation (47) is approximately $44 \mathrm{fsec}$, a value that is considerably less than the 200 fsec figure calculated using equations (11) - (15). Conversely, the height is overstated by roughly a factor of four. Further, the agreement is considerably worse than the rate equation pulse of Fig. 15. Nonetheless, that the inherently semiclassical set of (43) and (44) gives rise to 


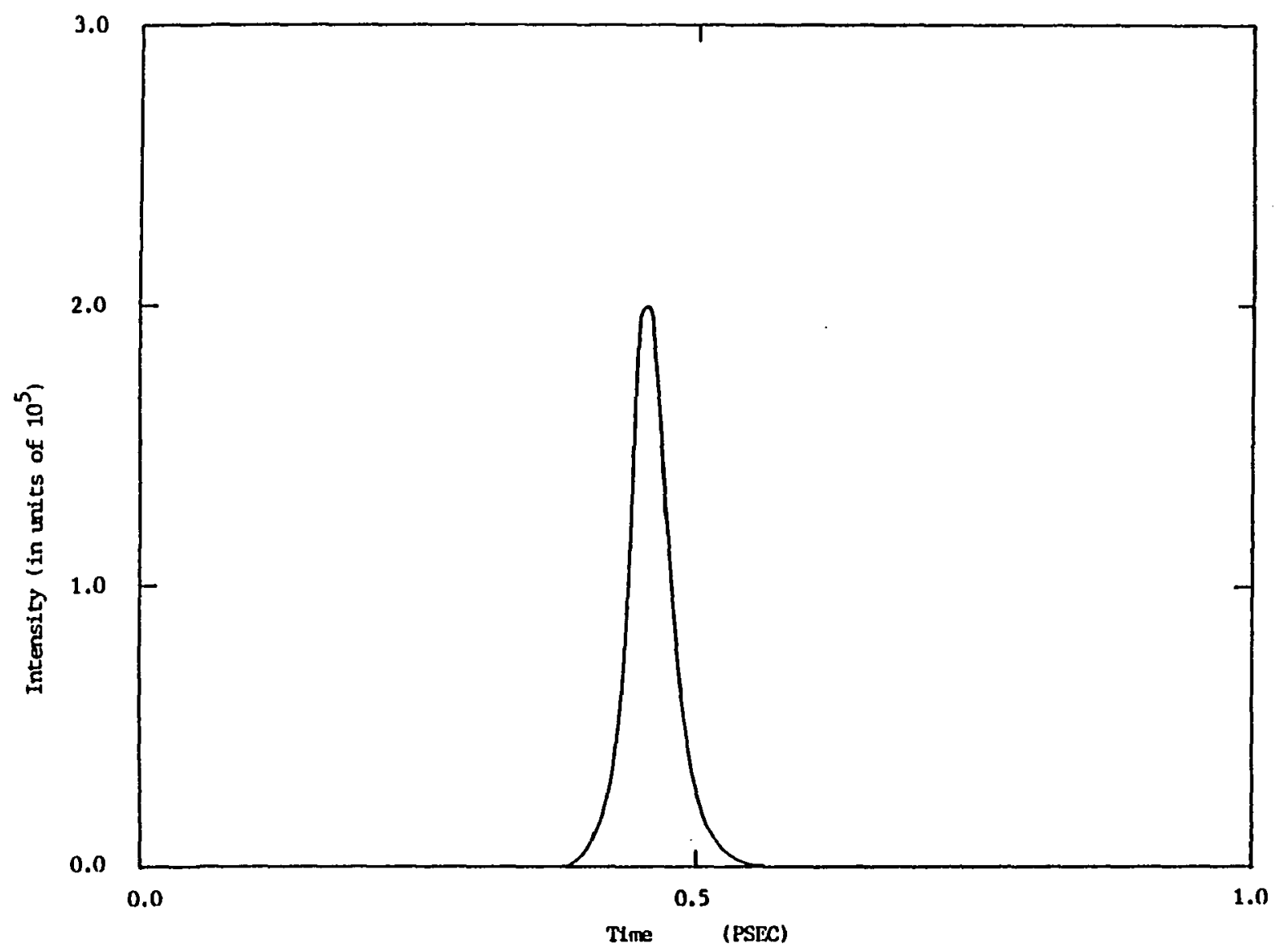

Figure 16. Analytic solution to the zero cavity length detuning equations.

$\Xi$ 
reasonable pulses is an indication of the importance of semiclassical effects in SPML operation, particularly at very small cavity length detunings.

If we ignore the effects of spontaneous emission, our solution for $I(t)$ may be written simply as:

$I(t)=\left(\tau_{2}(r-1)^{2} / 2 T_{s}\right) \operatorname{sech}^{2}\left\{(r-1)\left(t-T_{p k}\right) / T_{s}\right\}$

In equation (49), $\mathrm{T}_{\mathrm{pk}}$ is the time when the peak of the output pulse occurs. From equation (46) with $\mathrm{D}=1$ and $\mathrm{I}_{0}=0, \mathrm{~T}_{\mathrm{pk}}$ is:

$T_{p k}=\left(T_{s} / 2(r-1)\right) \ln (r)$

Equation (49) is an exact solution to our zero detuning equations, a fact that may easily be verified by direct substitution into equations (37) and (38). From equation (49) it follows that the FWHM pulse width in the zero detuning limit is approximately,

$t_{p}=(1.7627) T_{s} /(r-1)$

The hyperbolic secant form in equation (49) is a recurring one in the study of laser pulses. The popular formalism of Haus yields such a form for certain types of passively mode-locked lasers [10], and this result has been compared through experimental autocorrelation measurements with the output of a passively mode-locked dye laser [39]. A useful result given in reference [39] is the autocorrelation of a $\operatorname{sech}^{2}\left(t / t_{p}\right)$ pulse: 
$G(\tau)=\left\{\cosh \left(\tau / t_{p}\right)-\left(\tau / t_{p}\right) \sinh \left(\tau / t_{p}\right)\right\} / \sinh ^{3}\left(\tau / t_{p}\right)$

We note, however, that out formalism is fundamentally distinct from that of Haus, and we feel a better analogy may be drawn between ultrashort pulse production in zero detuned SPML dye lasers and the phenomenon of self-induced transparency. Self-induced transparency was well studied by McCall and Hahn [40-42] who also derived hyperbolic secant functions. In both self-induced transparency and in our modeling of SPML dye lasers, one seeks steady-state pulse solutions to inherently semiclassical Maxwell-Schrodinger equations.

\section{CONCLUSIONS}

We have explored a nonlinear dynamical model of a SPML dye laser simplified principally by considering a delta function pump. From a motivational standpoint, this is the regime where the latest SPML dye lasers operate $[7,8,27]$. Further approximations have led us to the interpretation that SPML dye lasers operated in the short pump pulse limit are predominantly gain switched devices. Thus, they can, in some sense, be described by the giant pulse laser equations derived in the early 1960 s [28-31]. We have also seen, however, that for quantitative agreement, it is important to consider, among other things, semiclassical effects. Hence, in the laser system discussed in this paper, one is $Q$-switching a coherent laser medium.

More specifically, we have distilled our model into two simple 
sets, the rate equation limit - equations (25) and (26) - and the zero detuning limit - equations (37) and (38). The distinction between these two sets lies in the time constant of the intensity equation and in the degree of nonlinearity. Though slightly different, both of these sets qualitatively describe the ultrashort pulse production of SPML lasers. Further simplification of either set results in an absence of pulsed solutions. This leads us to identify the nonlinear term, ID, common to the intensity equation of both sets, as a critical mode-locking term. Physically, then, it is a saturating, nonadiabatically changing population difference that gives rise to SPML laser pulses. Not surprisingly, it is the large rate constant associated with the intensity equation that allows ultrashort pulse durations.

The delta function limit is particularly important because, for a given dye and cavity, it defines the region of operation that gives the optimum, shortest output pulses. Furthermore, the ultrashort pump pulse provides degrees of freedom unique to synch-pumped systems; one does not have such optimization parameters in $\mathrm{cw}$ pumped colliding pulse mode-locked lasers $[43,44]$. One might speculate that hybridly $[45,46]$ mode-locked laser systems driven by pulses of a few picoseconds could produce output pulses that compare favorably with current colliding pulse mode-locked lasers. 


\section{CHAPTER IV}

\section{COHERENCE EFFECTS IN HYBRIDLY \\ MODE-LOCKED DYE LASERS: THEORY AND EXPERIMENT}

\section{INTRODUCTION}

Today's commercial mode-locked dye lasers offer tunable, subpicosecond pulses for a variety of applications, including high-speed time resolved spectroscopy and novel, precision photochemistry. These femtosecond sources come in three types. The shortest optical pulses directly from a laser come from the colliding pulse mode-locked laser, and in some cases these pulses are as short as $27 \mathrm{fsec}$ [47]. A synchronously pumped mode-locked (SPML) dye laser that is fully optimized with respect to the pump pulse will produce output pulses of a few hundred femtoseconds in duration $[7,27]$. An alternate technique for ultrashort laser pulse generation is the hybridly mode-locked laser, which is a synchronously pumped mode-locked laser with an added intracavity saturable absorber. This active-passive technique has produced optical pulses with durations of approximately 60 fsec [48-50]. It is the theory that governs these hybridly mode-locked dye lasers that is the subject of this chapter.

Considerable improvement has been made in the technology of hybridly mode-locked dye lasers since the first report appeared a decade ago [57]. In addition to reported pulses as short as $55 \mathrm{fsec}$ [49], subpicosecond operation has been demonstrated over the wide wavelength range of $560-840 \mathrm{~nm}$ using different gain and 
saturable absorber dyes [52]. One of the most important contributors to this development has been the understanding of the role of group velocity dispersion and the corresponding methods of chirp correction $[48,49,50,52]$. There have also been important advances in both active and passive (structural) cavity length stabilization techniques [46,53,54]. Another, less remarked upon, reason for these advances has been the higher powers available from improved pump sources.

In spite of the healthy state of the technology, there are several facets of hybridly mode-locked laser operation that could still be improved. For example, the wavelength range over which the shortest pulses are produced could be extended. Further, a blue hybridly mode-locked dye laser has not yet been reported. Most applications of these lasers would benefit from improved overall stability of the pulse train. Additionally, we see the possibility for further optimization of the systems through improvements of the pump sources.

In order to achieve these improvements it seems necessary to gain an enhanced understanding of the dynamics of the hybridly mode-locked process, particularly from a theoretical basis. To date there have only been a few theoretical studies of hybridly mode-locked laser operation $[54,55,56]$ and many theoretical issues remain unaddressed.

In selecting a theoretical approach for modeling a hybridly mode-locked dye laser, one may consider the wealth of work on SPML systems; a hybridly mode-locked dye laser can be viewed as a synchronously pumped mode-locked dye laser with a saturable absorber. The theoretical understanding of SPML lasers has advanced on both the analytic and the numerical levels. Because of the stringent approximations imposed in any analytic theory, 
the two approaches are perhaps best considered as distinct, at least in their goals. The analytic work has evolved from the self-consistent pulse picture of Kuizenga and Siegman [9] and of Haus [10] and specific application of this formalism to SPML systems has been considered by Frigo et. al. [11], Ausschnitt et. al. [12] and Kim et. al. [13]. These studies have provided closed-form, approximate expressions for the output pulse characteristics as functions of cavity length, system gain and several dye parameters. The added complexity of the additional saturable absorber in a hybridly mode-locked laser makes it difficult to extend these limited analyses to the case at hand. Further, the validity of these theories has recently been challenged in the literature [57].

More rigorous numerical modeling of SPML dye lasers has been undertaken by many groups [14-24] since only this technique affords the study of transient effects and specific pulse shape information. The numerical models also have the advantage that their framework can easily support the study of random noise sources such as spontaneous emission which can lead to profound effects at large cavity mismatches, far from the optimum cavity length $[20,21]$. Much of this computational work has been done in the rate equation limit, however semiclassical effects have been shown to be significant in the steady state pulse shape [21-24]. The few theoretical studies of hybridly mode-locked lasers have all been numerical studies taken in the rate equation limit $[54,55,56]$. In light of the results of reference [24], we have included coherence effects in this theory.

Section il of this chapter contains a derivation of the nonlinear dynamical equations that govern a hybridly mode-locked dye laser. Section III describes experiments performed 
on a commercially available hybridly mode-locked dye laser. The model is therefore verified by direct comparison of measured and calculated autocorrelations taken over a wide range of cavity detunings. Section IV presents the results of several pertinent theoretical explorations of the model. Included here are effects of varying the absorber molarity and the saturation parameter. These parameters are important because they can, to some extent, be varied experimentally in order to optimize performance for a given application. The model is general enough to allow for the numerical study of semiclassical and dipole orientational effects, and discussions of these points are also included here. Finally, we consider the pump pulse width and power as additional optimization parameters. The underlying goal of this paper is to provide a better understanding of hybridly mode-locked lasers and, in turn, to suggest ways to improve the performance of existing hybridly mode-locked systems.

\section{SEMICLASSICAL MODEL}

Our goal in this section is to derive a model of a hybridly mode-locked dye laser. This section closely follows the work in reference [24], in which a coherent model for a SPML dye laser was derived. The work in reference [24] and below follow as an extension of Lamb's semiciassical laser theory [58]. This Maxwell-Schrodinger model has the advantage of providing a tractable formalism that accurately accounts for the vectoral nature of the electric field. It is also important to include semiclassical effects in problems where an electric field or a polarization is expected to fluctuate rapidly $[24,37,58]$. Below we derive the specialized density matrix equations that govern a dye 
laser amplifier and a saturable absorber. We also derive the Maxwell wave equation appropriate to the problem of a mode-locked dye laser. The driving terms in this electric field equation are the field induced polarizations. Thus the two sets are fully coupled. A proper choice of boundary conditions accounts for the laser cavity and synchronous pumping. The model is simplified as much as possible without losing any term that is expected to play an important role in the laser dynamics on a subpicosecond time scale. Our end result is a set of six coupled, nonlinear, ordinary integro-differential equations that govern the interplay of the electric field with the molecular populations and the polarizations of both the gain dye and the saturable absorber.

\section{DENSITY MATRIX EQUATIONS}

The energy level scheme used in this analysis is shown in Fig. 17. The four level amplifier system and much of the notation associated with it is taken directly from references $[24,33 \& 59]$. The pump absorption takes place between levels 0 and 3 , while the signal-stimulated transition occurs between levels 2 and 1. The molecules in level 3 decay nonradiatively into the upper lasing state, level 2, with the vibrational intraband relaxation time I3. $_{3}$ The molecules decay out of the lower lasing state, level 1 , into the ground state, level 0 , with the vibrational relaxation time $\tau_{1}$. The spontaneous decay time for the laser transition is represented by $\tau_{2}$. The actual energy level structure is more complex than this simple model would indicate. Nonetheless, the model does, at least, include the essential features of vibrational relaxation into and out of the laser levels. The saturable absorber is taken simply as 


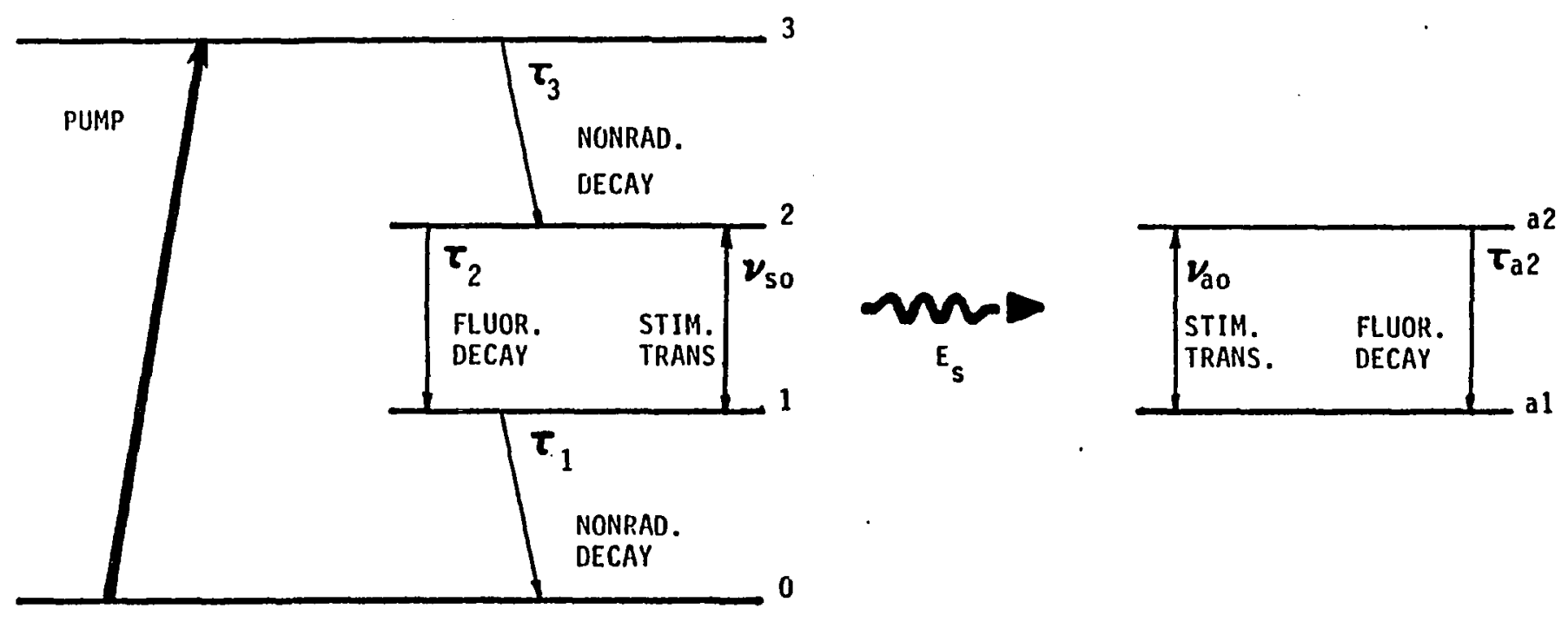

Eigure 17. Energy level diagram for hybridly mode-locked laser. 
a two level system that is resonant with the stimulated transition of the gain levels. The stimulated transition of the saturable absorber takes place between the ground state, level al, and the upper state, level a2. The spontaneous emission associated with the saturable absorber is characterized by the time constant, $\tau_{\mathrm{a} 2}$.

The procedure for setting up the density matrix equations is well known, and for the particular energy level scheme of this problem, the dynamical equations are [24,59]:

$$
\begin{aligned}
& \delta p_{00} / \delta t=-(i 2 \pi / h)\left(\rho_{03} \mu_{30}-\rho_{30} \mu_{03}\right) \cdot \mathbf{E}_{p}+\rho_{11 / \tau_{1}} \\
& \delta \rho_{33 / \delta t}=(i 2 \pi / h)\left(\rho_{03} \mu_{30}-\rho_{30} \mu_{03}\right) \cdot \mathbf{E}_{\mathbf{p}}-\rho_{33 / \tau_{3}} \\
& \delta p_{22 / \delta t}=-(i 2 \pi / h)\left(\rho_{21} \mu_{12}-\rho_{12} \mu_{21}\right) \cdot E_{s}-p_{22} / \tau_{2}+p_{33} / \tau_{3} \\
& \delta \rho_{11 / \delta t}=(i 2 \pi / h)\left(\rho_{21} \mu_{12}-\rho_{12} \mu_{21}\right) \cdot E_{s}-\rho_{11 / \tau_{1}}+\rho_{22} / \tau_{2} \\
& \delta_{p_{30} / \delta t}=-i \omega_{p 0} P_{30}+(i 2 \pi / h)\left(\rho_{00}-\rho_{33}\right) \mu_{30} \cdot E_{p}-p_{30} / T_{p} \\
& \delta p_{21} / \delta t=-j \omega_{s o} p_{21}-(i 2 \pi / h)\left(p_{22}-\rho_{11}\right) \mu_{21} \cdot \mathbf{E}_{S}-p_{21} / T_{S} \\
& \text { P03 }=030^{*} \\
& p_{12}=p_{21}^{*} \\
& \delta \rho_{a 22} / \delta t=-(i 2 \pi / h)\left(\rho_{a 21} \boldsymbol{\mu}_{a 12}-\rho_{a 12} \boldsymbol{\mu}_{\mathbf{a} 21}\right) \cdot \mathbf{E}_{\mathbf{s}}-\rho_{a 22 / \tau} \tau_{a 2}
\end{aligned}
$$




$$
\begin{aligned}
& \delta \rho_{a 11 / \delta t}=(i 2 \pi / h)\left(\rho_{a 21} \mu_{a 12}-\rho_{a 12} \mu_{a 21}\right) \cdot \mathbf{E}_{\mathbf{S}}+\rho_{a 22 / \tau_{a 2}} \\
& \delta \rho_{a 21 / \delta t}=-i w_{\text {aoo }} \rho_{a 21}-(i 2 \pi / h)\left(\rho_{a 22}-\rho_{a 11}\right) \mu_{a 21} \cdot \mathbf{E}_{\mathbf{s}}-\rho_{a 21 / T} \\
& \rho_{a 12}=\rho_{a 21}{ }^{*}
\end{aligned}
$$

Equations (53) - (60) govern the molecular dynamics of the four level gain system and are identical to equations (1) - (8) in reference [24]. Equations (61) - (64) are the dynamical equations of motion for the density matrix elements of the saturable absorber. $\mathbf{E}_{\mathbf{p}}$ and $\mathbf{E}_{\mathbf{s}}$ are, respectively, the pump and signal electric fields, while $\omega_{\text {po }} \omega_{\mathrm{so}}$ and $\omega_{\text {ao }}$ are, respectively, the center frequencies of the pump, signal and absorbing transitions. The various $\boldsymbol{\mu}_{\mathbf{j i}}$ are the matrix elements of the dipole moment operators. The coherence times for the off-diagonal matrix elements are $T_{p}, T_{s}$ and $\mathrm{T}_{\mathrm{a}}$.

It is convenient to multiply equations (57), (58) and (63) by $\mu_{03}, \mu_{12}$ and $\mu_{a 12}$ respectively, and the results are:

$$
\begin{aligned}
& \delta \eta 30 / \delta t=-i \omega_{p o} \eta_{30}+(i 2 \pi / h)\left(\rho_{00}-\rho_{33}\right) \mu_{p} L^{2}\left(E_{p} \cdot e_{p}\right) e_{p}-\eta 30 / T_{p} \\
& \delta \eta_{21} / \delta t=-i \omega_{S O} \eta_{21}-\left.(i 2 \pi / h)\left(\rho_{22}-\rho_{11}\right) \mu_{S}\right|^{2}\left(E_{S} \cdot e_{S}\right) e_{S}-\eta_{21} / T_{S} \\
& \delta \eta_{\mathbf{a} 21} / \delta t=-i \omega_{a 0} \eta_{\mathbf{a} 21}-(i 2 \pi / h)\left(p_{a 22}-p_{a 11}\right) \mu_{\mathbf{a}} L^{2}\left(\mathbf{E}_{\mathbf{s}} \cdot e_{\mathbf{a}}\right) e_{\mathbf{a}} \\
& \text { - } \pi_{221 / T_{a}}
\end{aligned}
$$


The unit vectors in the direction of $\mu_{03}, \mu_{12}$ and $\mu_{\mathbf{a} 12}$ are $\mathbf{e}_{\mathbf{p}}, \mathbf{e}_{\mathbf{s}}$ and $e_{\mathbf{a}}$ respectively. The other new parameters are:

$\eta_{30}=\rho_{30 \mu_{03}}=\rho_{30 \mu_{03} e_{p}}$

$\eta_{21}=\rho_{21} \mu_{12}=\rho_{21} \mu_{12} e_{3}$

$\eta_{\mathbf{a} 21}=\rho_{\mathrm{a} 21} \mu_{\mathbf{a} 12}=\rho_{\mathrm{a} 21} \mu_{\mathrm{a} 12} e_{\mathbf{a}}$

$\left|\mu_{p}\right|^{2}=\mu_{30} \mu_{30}{ }^{*}=\mu_{30} \mu_{03}$

$\left|\mu_{s}\right|^{2}=\mu_{21} \mu_{21}^{*}=\mu_{21} \mu_{12}$

$\left|\mu_{a}\right|^{2}=\mu_{a 21} \mu_{a 21}{ }^{*}=\mu_{a 21} \mu_{a 12}$

Equations (53) - (56), (61), (62) and (65) - (67) describe the response of the two organic dye media to an arbitrary electric field. These equations can be simplified substantially for the case of a hybridly mode-locked dye laser by specifying plane-wave pump and signal fields having parallel propagation vectors $\mathbf{k}_{\mathbf{p}}$ and $\mathbf{k}_{\mathbf{s}}$. For propagation in the $\mathbf{z}$ direction, the field components can be written as:

$\mathbf{E}_{\mathbf{p}}(z, t)=y_{2} \mathbf{E}_{\mathbf{p}}^{\prime}(z, t) \exp \left[i\left(k_{p} z-\omega_{p} t\right)\right]+c . c$.

$E_{S}(z, t)=y_{2} E_{s}^{\prime}(z, t) \exp \left[i\left(k_{s} z-w_{s} t\right)\right]+c . c$. 
where $\mathbf{E}_{\mathbf{p}}(z, t)$ and $\mathbf{E}_{\mathbf{s}}(z, t)$ are slowly varying complex vector functions of $z$ and $t$. This form for the electric field is rigorously valid for a ring dye laser. Many of the results described below should hold for the hybridly mode-locked laser since the mode-locked pulse, which, by definition, is short compared to the cavity length, never counter-propagates through either of the active media. This is clearly not the experimental situation of the colliding pulse mode-locked laser.

The plane-wave dependence in the off-diagonal matrix elements can be factored out using the substitutions

$\eta_{30}(\theta, \phi, z, t)=\eta_{p}(\theta, \phi, z, t) \exp \left[i\left(k_{p} z-\omega_{p} p^{t}\right)\right] \mathbf{e}_{p}$

$\eta_{21}(\theta, \phi, z, t)=\eta_{s}(\theta, \phi, z, t) \exp \left[i\left(k_{s} z-w_{s} t\right)\right] e_{s}$

$\eta_{\mathbf{a} 21}(\theta, \phi, z, t)=\eta_{\mathbf{a}}(\theta, \phi, z, t) \exp \left[i\left(k_{\mathbf{s}} z-w_{s} t\right)\right] e_{\mathbf{a}}$

In equation (76), $\theta$ measures the orientation of a class of pump dipoles with respect to the $\mathrm{x}$ axis (which is perpendicular to the pump direction), and $\phi$ measures the orientation of the dipoles around the $\mathrm{x}$ axis $[24,59]$. Similar interpretations hold for the gain dipoles in equation (77) and the saturable absorber dipoles in equation (78).

When equations (74) - (78) are substituted into our set of equations (53) - (56), (61), (62) and (65) - (67) and the rotating wave approximation is used, our set of density matrix equations simplify to:

$$
\delta \rho_{00 / \delta t}=-(2 \pi / h) \operatorname{Im}\left(\boldsymbol{\eta}_{\mathbf{p}} \cdot \mathbf{E}_{\mathbf{p}}^{*}\right)+\rho_{11 / \tau_{1}}
$$


$\delta \rho_{33 / \delta t}=(2 \pi / h) \operatorname{Im}\left(\eta_{\mathbf{p}} \cdot \mathbf{E}_{\mathbf{p}}^{\prime}\right)-\rho_{33 / \tau_{3}}$

$$
\delta \rho_{22 / \delta t}=(2 \pi / h) \operatorname{Im}\left(\boldsymbol{n}_{\mathbf{s}} \cdot \mathbf{E}_{\mathbf{s}}^{\prime}\right)-\rho_{22 / \tau_{2}}+\rho_{33 / \tau_{3}}
$$

$$
\delta \rho_{11 / \delta t}=-(2 \pi / h) \operatorname{Im}\left(\eta_{\mathbf{s}} \cdot \mathbf{E}_{\mathbf{S}}^{*}\right)-\rho_{11 / \tau_{1}}+\rho_{22 / \tau_{2}}
$$

$$
\delta \rho_{\mathbf{a} 22 / \delta t}=(2 \pi / h) \operatorname{Im}\left(\eta_{\mathbf{a}} \cdot \mathbf{E}_{\mathbf{s}}^{*}\right)-\rho_{\mathrm{a} 22 / \tau_{\mathbf{a} 2}}
$$

$$
\left.\delta \rho_{a 11 / \delta t}=-(2 \pi / h) \operatorname{Im}\left(\eta_{\mathbf{a}} \cdot E_{\mathbf{s}}^{\prime}\right)^{\mathbf{z}}\right)+\rho_{\mathbf{a} 22 / \tau_{\mathbf{a} 2}}
$$

$$
\delta \eta_{p} / \delta t=i\left(\omega_{p}-\omega_{p o}\right) \eta_{p}+(i \pi / h)\left(p_{00}-p_{33}\right) \mu_{p} L^{2}\left(E_{p}^{*} \cdot e_{p}\right)-\eta_{p} / T_{p}
$$

$\delta \eta_{S / \delta t}=i\left(\omega_{S}-\omega_{S O}\right) \eta_{S}-\left.\left.(i \pi / h)\left(\rho_{22}-\rho_{11}\right)\right|_{S}\right|^{2}\left(E_{s}^{\prime} \cdot e_{3}\right)-\eta_{S} / T_{S}$

$$
\delta \eta_{a} / \delta t=i\left(\omega_{s}-\omega_{a 0}\right) \eta_{a}-\left.(i \pi / h)\left(p_{a 22}-\rho_{a 11}\right) \mu_{a}\right|^{2}\left(E_{s}^{\prime} \cdot e_{a}\right)-\eta_{a} / T_{a}
$$

where $n_{p}=n_{p} e_{p}, n_{s}=n_{s} e_{s}$ and $n_{a}=n_{a} e_{\mathbf{a}}$.

The average values of the polarizations induced by the pump and signal fields are given by,

$$
\begin{aligned}
& \mathbf{P}_{\mathbf{p}}=\int_{\Omega} \mathrm{n}_{\mathrm{p}}(\theta, \phi)\left(\rho_{03} \mu_{30}+\rho_{30} \mu_{03}\right) d \Omega \\
& \mathbf{P}_{\mathbf{s}}=\int_{\Omega} \mathrm{n}_{\mathrm{s}}(\theta, \phi)\left(\rho_{21} \boldsymbol{\mu}_{12}+\rho_{12} \boldsymbol{\mu}_{21}\right) d \Omega
\end{aligned}
$$


$\mathbf{P}_{\mathbf{a}}=\int_{\Omega} \mathrm{n}_{\mathbf{a}}(\theta, \phi)\left(\rho_{\mathrm{a} 21} \boldsymbol{\mu}_{\mathbf{a} 12}+\rho_{\mathrm{a} 12} \boldsymbol{\mu}_{\mathbf{a} 21}\right) \mathrm{d} \Omega$

where $n_{p}(\theta, \phi) d \Omega, n_{s}(\theta, \phi) d \Omega$ and $n_{a}(\theta, \phi) d \Omega$ are, respectively, the number of pump, gain and saturable absorber molecules per unit volume oriented within the solid angle $d \Omega$ about the $(\theta, \phi)$ direction. With the previous definitions, these polarizations can be written as

$\mathbf{P}_{\mathbf{p}}=\int_{\Omega} n_{p}(\theta, \phi) \eta_{p}(\theta, \phi, z, t) e_{p} d \Omega \exp \left[i\left(k_{p} z-\omega_{p} t\right)\right]+$ c.c.

$P_{s}=\int_{\Omega} n_{s}(\theta, \phi) \eta_{s}(\theta, \phi, z, t) e_{s} d \Omega \exp \left[i\left(k_{s} z-\omega_{s} t\right)\right]+c . c$.

$\mathbf{P}_{\mathbf{a}}=\int_{\Omega} n_{\mathbf{a}}(\theta, \phi) n_{\mathbf{a}}(\theta, \phi, z, t) e_{\mathbf{a}} d \Omega \exp \left[i\left(k_{s} z-\omega_{s} t\right)\right]+$ c.c.

Now, equations (79) - (87) and (91) - (93) may be taken as a complete set implying the pump, the gain and the saturable absorber polarization components in terms of the pump and signal fields. The next step in this analysis is to develop equations which express the fields in terms of the polarizations.

\section{MAXWELL'S EQUATIONS}

From Maxwell's equations, the pump and signal vectors are related to the corresponding polarizations by the wave equations

$\delta^{2} \mathbf{E}_{\mathbf{p} / \delta z^{2}}-\mu \epsilon \delta^{2} \mathbf{E}_{\mathbf{p} / \delta t^{2}}-\mu \sigma \delta \mathbf{E}_{\mathbf{p} / \delta t}=\mu \delta^{2} \mathbf{P}_{\mathbf{p} / \delta t^{2}}$ 


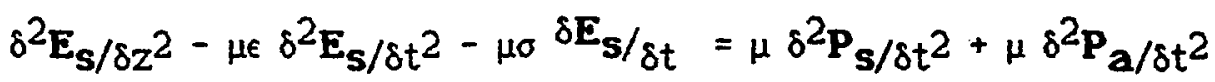

Using equations (74), (75), (88), (89) and (90) these wave equations reduce to

$$
\begin{aligned}
& \delta \mathbf{E}_{\mathbf{p} / \delta \mathbf{z}}^{\prime}+(1 / \mathrm{v}) \delta \mathbf{E}^{\prime} \mathbf{p} / \delta t+\left(\gamma_{p} / 2\right) \mathbf{E}_{\mathbf{p}}^{\prime} \\
& =\left(i \mu \omega_{p}{ }_{p}^{2} / k_{p}\right) \int_{\Omega} n(\theta, \phi) \eta_{p} e_{p} d \Omega \\
& \delta E_{s / \delta z}^{\prime}+(l / v) \delta E_{s / \delta t}^{\prime}+\left(v_{s} / 2\right) E_{s}^{\prime} \\
& =\left(j \mu \omega_{s} 2 / k_{S}\right) \int_{\Omega} n(\theta, \phi) \eta_{s} e_{s} d \Omega \\
& +\left(i_{\mu \omega} \omega_{s} 2 / k_{s}\right) \int_{\Omega} n_{a}(\theta, \phi) n_{a} e_{a} d \Omega
\end{aligned}
$$

In equations (96) and (97), the higher order space and time derivatives of slowly varying quantities have been neglected, and the rotating-wave approximation has been employed. The propagation constants are given by $\mathrm{k}_{\mathrm{p}}{ }^{2}=\mu \epsilon \omega_{\mathrm{p}}{ }^{2}$ and $\mathrm{k}_{\mathrm{s}}{ }^{2}=\mu \epsilon \omega_{\mathrm{s}}{ }^{2}$. The approximate velocity $v=(\mu \epsilon)^{-1 / 2}$ has also been introduced.

\section{SIMPLIFICATIONS AND SPECIALIZATIONS}

Equations (79) - (87), (96) and (97) together form a complete set for investigating the interaction of the laser pump and signal fields with gain and absorbing dye media. These equations are more general than necessary for the present application of a hybridly mode-locked laser. We now make several simplifications 
that do not seem to compromise the model's agreement with experiment.

If the pump and signal frequencies are close to the center frequencies of the molecular transitions $\left(w_{p} \approx w_{\text {po }}, w_{\mathrm{S}} \approx w_{\mathrm{sO}}, w_{\mathrm{S}} \approx\right.$ $\left.w_{a 0}\right)$ and the saturation of the pump transition is weak $\left(1 \approx p_{00} \gg\right.$ P33) then equations (85), (86) and (87) become:

$$
\delta \eta_{p} / \delta t=\left.(i \pi / h) \mu_{p}\right|^{2}\left(E_{p}^{*} \cdot e_{p}\right)-\eta_{p} / T_{p}
$$

$\delta \eta_{S} / \delta t=-(i \pi / h)\left(\rho_{22}-\rho_{11}\right)\left|\mu_{S}\right|^{2}\left(E_{s}^{*} \cdot e_{s}\right)-\eta_{s} / T_{S}$

$$
\delta \eta_{a} / \delta t=-(i \pi / h)\left(\rho_{a 22}-\rho_{a 11}\right)\left|\mu_{a}\right|^{2}\left(E_{s}^{*} \cdot e_{a}\right)-\eta_{a} / T_{a}
$$

These approximations would not always be valid, but they seem to provide adequate agreement with available data. If necessary, the more general equations could be retained. In particular, we note that issues of frequency detuning can be addressed by generalizing the appropriate coherence time to a complex quantity whose imaginary part is precisely the difference between the field frequency and the transition center frequency.

As will be discussed below, practical values for the coherence times, $T_{p}$ and $T_{S}$ are on the order of $10^{-14} \mathrm{sec}$. On the other hand, the width of the pump pulses for hybridly mode-locked lasers are generally about $10^{-11} \mathrm{sec}$. Thus, the time derivative in (98) may be neglected as $n_{p}$ instantaneously tracks the value of the pump field amplitude. Explicitly, the steady state solution of equation (98) is: 
$\eta_{p}=\left({ }^{(\pi T} T_{p / h}\right)\left|\mu_{p}\right|^{2} \mathbf{E}_{p}^{\prime} \cdot e_{p}$

The dye laser pulse is much shorter than the pump pulse and it is not obvious that the corresponding derivatives in equations (99) and $(100)$ can be neglected. In fact it will be shown that neglect of this derivative - the rate equation limit - can lead to significant errors in the predictions of the theory.

The width of the pump pulse can also be shown to be long compared to the vibrational relaxation time ${ }_{3}$. Therefore, the time derivative in equation (80) is unimportant and we may adiabatically eliminate $p_{33}$ from our set of equations. Using the steady state solution to (80) and equation (100),

$\rho_{33}=\left.\left(2 \pi^{2} \tau_{3} T_{p} / h^{2}\right) \mu_{p}\right|^{2}\left|E_{p}^{\prime} \cdot e_{p}\right|^{2}$

Substitution of equation (102) into equation (81) yields

$$
\begin{array}{r}
\delta \rho_{22 / \delta t}=(2 \pi / h) I m\left(\eta_{s} \cdot E_{s}^{\prime}\right)-\rho_{22 / \tau_{2}+} \\
\left(2 \pi^{2} T_{p} / h^{2}\right)\left|\mu_{p}\right|^{2}\left|E_{p}^{\prime} \cdot e_{p}\right|^{2}
\end{array}
$$

As long as pump depletion is not too severe, the field, $\mathbf{E}_{\mathbf{p}}$ may be regarded as a known quantity, and equations (82), (83), (84), (97), (99), (100) and (103) form a set sufficient to characterize a hybridly mode-locked laser.

It can now be assumed that $\mathbf{E}_{\mathbf{p}}^{\prime}$ and $\mathbf{E}_{\mathbf{s}}^{\prime}$ are vectors that are purely real, while $\eta_{s}$ and $\eta_{\mathbf{a}}$ are purely imaginary. With the substitutions, $\eta_{\mathbf{s}}=$ in ${ }_{\mathbf{s}}$ and $\eta_{\mathbf{a}}=$ in $\boldsymbol{\eta}_{\mathbf{a}}$, our set of equations reduces 
to the seven purely real equations:

$$
\begin{aligned}
& \delta_{\rho_{22} / \delta t}=(2 \pi / h) \eta_{S}^{\prime} \mathbf{E}_{\mathbf{s}}^{\prime} \cdot \boldsymbol{e}_{\mathbf{S}}-\rho_{22 / \tau_{2}} \\
& +\left.\left(2 \pi^{2} T_{p} / h^{2}\right) \mu_{p}\right|^{2}\left(E_{p}^{*} \cdot e_{p}\right)^{2} \\
& \delta \rho_{11 / \delta t}=-(2 \pi / h) \eta_{S}^{\prime} E_{s}^{\prime} \cdot e_{s}-P_{11 / \tau_{1}}+P_{22} / \tau_{2} \\
& \delta \eta_{S}^{\prime} / \delta t=-(\pi / h)\left(\rho_{22}-\rho_{11}\right) \mu_{S}{ }^{2}\left(E_{s}^{0} \cdot e_{s}\right)-\eta_{S}^{\prime} / T_{S} \\
& \delta E^{\prime} s_{/ \delta z}+(1 / v) \delta E^{\prime} s / \delta t+\left(\gamma_{S} / 2\right) E_{s}^{\prime} \\
& =-\left(\mu \omega_{s} 2 / k_{s}\right) \int_{\Omega} n(\theta, \phi) \eta_{s}^{\prime} e_{s} d \Omega \\
& -\left(\mu w_{s}{ }^{2} / k_{s}\right) \int_{\Omega} n_{a}(\theta, \phi) \eta_{a} e_{a} d \Omega \\
& \delta p_{a 22 / \delta t}=(2 \pi / h) \eta_{a}^{\prime} E_{s}^{*} \cdot e_{\mathbf{a}}-p_{a 22 / \tau_{a 2}} \\
& \delta p_{a 11 / \delta t}=-(2 \pi / h) \eta_{a}^{\prime} \mathbf{E}_{s}^{\prime} \cdot e_{\mathbf{a}}+\rho_{a 22 / \tau_{a 2}} \\
& \delta \eta_{a}^{\prime} / \delta t=-(\pi / h)\left(\rho_{a 22}-\rho_{a 11}\right) \mu_{a}{ }^{2}\left(E_{s}^{\prime} \cdot e_{a}\right)-\eta_{a}^{\prime} / T_{a}
\end{aligned}
$$

In most dye molecules, the absorption and emission dipoles are parallel $\left(\mathbf{e}_{\mathbf{p}}=\mathbf{e}_{\mathbf{s}}\right)$. Also, in most mode-locked dye lasers, the pump and signal field polarizations are parallel to each other, and it is assumed here that these fields are parallel to the $\mathrm{x}$-axis. Thus $\mathbf{E}_{\mathbf{s}}^{\prime} \cdot \boldsymbol{e}_{\mathbf{s}}=E_{S}^{\prime} \cos \theta_{S}, \mathbf{E}_{\mathbf{p}}^{\prime} \cdot \boldsymbol{e}_{\mathbf{p}}=E_{p}^{\prime} \cos \theta_{S}$ and $\mathbf{E}_{\mathbf{s}}^{\prime} \cdot \boldsymbol{e}_{\mathbf{a}}=E_{S}^{\prime} \cos$ 
$\theta_{a}$. Here, $E_{S}^{\prime}$ and $E_{p}^{\prime}$ represent the amplitudes of the corresponding vector electric fields. We now choose the isotropic distribution as the specific dye molecule orientational distribution $[33,59]$. Thus, $n(\theta, \phi)=N_{s} / 4 \pi$ and $n_{a}(\theta, \phi)=N_{a} / 4 \pi$. In spherical coordinates, the differential solid angle is $d \Omega=\sin (\theta) d \theta d \phi$. With these conditions, and introducing the variables $\mathrm{x}=\cos \theta_{\mathrm{S}}$ and $\mathrm{y}=$ $\cos \theta_{a}$, the vector set (104) - (110) becomes the scalar set:

$$
\begin{aligned}
& \delta_{\rho 22 / \delta t}=(2 \pi / h) \pi_{S}^{\prime} E_{S}^{\prime} x-\rho_{22} / \tau_{2}+\left(2 \pi^{2} T_{p} / h^{2}\right) \mid \mu_{p} P^{2}\left(E_{p}^{\prime}\right)^{2} x^{2} \\
& \delta \rho_{11 / \delta t}=-(2 \pi / h) \eta_{S}^{\prime} E_{S}^{\prime} x-\rho_{11 / \tau_{1}}+\rho_{22 / \tau_{2}} \\
& \delta \eta_{S}^{\prime} / \delta t=-(\pi / h)\left(\rho_{22}-p_{11}\right) \|\left._{\mu_{S}}\right|^{2} E_{S}^{\prime} x-\eta_{S}^{\prime} / T_{S} \\
& \delta E_{S / \delta Z}^{\prime}+(1 / V) \delta E_{S / \delta t}^{\prime}+\left(\Upsilon_{S} / 2\right) E_{S}^{\prime} \\
& =-\left(\mu \omega_{s}^{2} / k_{s}\right) N_{S} \int_{0}^{1} \eta_{s}^{\prime} x d x \\
& -\left(\mu \omega_{s}^{2} / k_{s}\right) N_{a} \int_{0}^{1} \eta_{a}^{\prime} y d y \\
& \delta \rho_{a 22 / \delta t}=(2 \pi / h) \eta_{a}^{\prime} E_{s}^{\prime} Y-\rho_{a 22 / \tau_{a 2}} \\
& \delta \rho_{a 11} / \delta t=-(2 \pi / h) \eta_{a}^{\prime} E_{s}^{\prime} y+\rho_{a 22} / \tau_{a 2} \\
& \delta \eta_{a / \delta t}^{\prime}=-(\pi / h)\left(\rho_{a 22}-\rho_{a 11}\right) \mu_{a} a^{2} E_{s}^{\prime} y-\eta_{a}^{\prime} / T_{a}
\end{aligned}
$$


Equations (111) - (117) can be written in a compact form if one introduces suitably normalized forms for the dependent variables. We choose to write the field in terms of its steady state gain saturating value. With this philosophy, the choices adopted here include:

$$
\begin{aligned}
& A=(2 \pi / h)\left|\mu_{S}\right|\left(\tau_{2} T_{S / 2}\right)^{1 / 2} E_{S}^{\prime} \\
& D=\left(\mu \omega_{S} 2 / \gamma_{S} k_{S}\right)\left(2 \pi T_{S} \mid \mu_{S} I^{2} N_{S / h}\right)\left(\rho_{22}-\rho_{11}\right)
\end{aligned}
$$

$M=\left(\mu \omega_{s}{ }^{2} / \gamma_{S} k_{s}\right)\left(2 \pi T_{s} \mid \mu_{s}{ }^{2} N_{S} / h\right)\left(\rho_{22}+\rho_{11}\right)$

$Q=-\left(\mu \omega_{s} 2 / \gamma_{S} k_{s}\right)\left(2 \pi\left|\mu_{S}\right| N_{S} / h\right)\left(2 \tau_{2} T_{S}\right)^{1 / 2} \eta_{S}^{\prime}$

$D_{a}=\left(\mu w_{s}{ }^{2} / \gamma_{s} k_{s}\right)\left(2 \pi T_{a} \mid \mu_{s}{ }^{2} N_{a / h}\right)\left(p_{a 22}-p_{a 11}\right)$

$Q_{a}=-\left(\mu \omega_{s} 2 / \gamma_{s} k_{s}\right)\left(2 \pi\left|\mu_{s}\right| N_{a} / h\right)\left(2 \tau_{2} T_{s}\right)^{y / 2} \eta_{a}$

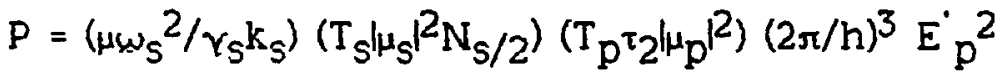

Thus, $\mathrm{A}$ is a normalized field, $\mathrm{D}$ is a normalized population difference for the gain, $M$ is a normalized population sum for the gain, $Q$ is a normalized polarization for the gain, $D_{a}$ is a normalized population difference for the saturable absorber, $Q_{a}$ is a normalized polarization for the saturable absorber and $P$ is a normalized pump rate. Since equations (115) aand (116) are not independent, we can express the saturable absorber population 
sum as a constant,

$\alpha=\left(\mu_{s}{ }^{2} / \gamma_{s} K_{s}\right)\left(2 \pi T_{a} \mid \mu_{a}{ }^{2} N_{a} / h\right)\left(\rho_{a 22}+\rho_{a 11}\right)$

In our calculations below, we have expressed $a$ in terms of the experimental small signal absorption of the saturable absorber dye jet. In terms of these new variables our governing set of equations become:

$\delta D / \delta t=-\left(1 / \tau_{2}\right)\left[\left(1+\tau_{2} / 2 \tau_{1}\right) D+\left(1-\tau_{2} / 2 \tau_{1}\right) M+2 Q A x-P(t) x^{2}\right]$

$\delta M / \delta t=-\left(1 / \tau_{2}\right)\left[-\left(\tau_{2} / 2 \tau_{1}\right) D+\left(\tau_{2} / 2 \tau_{1}\right) M-P(t) x^{2}\right]$

$\delta Q / \delta t=-\left(1 / T_{S}\right)[Q-A D x]$

$\delta A / \delta z+(1 / v) \delta A / \delta t=-\left(\gamma_{S} / 2\right)\left[A-\int_{0}^{1} Q x d x-\int_{0}^{1} Q_{a} y d y\right]$

$\delta D_{a} / \delta t=-\left(1 / \tau_{a 2}\right)\left[D_{a}+\alpha+2 \beta Q_{a} A y\right]$

$\delta Q_{a} / \delta t=-\left(1 / T_{a}\right)\left[Q_{a}-A D_{a} Y\right]$

The additional constant $\beta=f\left(T^{T}{ }^{\top} a 2^{\epsilon_{a}} / T_{s}{ }^{\top} \epsilon_{S}\right)$ accounts for the difference in saturation intensities and the possible difference in beam spot size, $f$, in the two dye media. 


\section{SYNCHRONOUS PUMPING}

Equations (126) - (131) may be used to describe the propagation of a short pulse of light interacting with a rather general dye laser amplifier/saturable absorber pair. These equations should have direct application to the more advanced mode-locked pulse amplification schemes which involve interwoven stages of amplifier and saturable absorber dyes empirically designed to amplify in energy and trim in pulse width [60,61].

of interest in this work is the application of equations (126) (131) to the case of a hybridly mode-locked dye laser. To this end, we now consider the specific problem of a synchronously pumped ring dye laser oscillator with a saturable absorber included in the cavity. In such a laser, the signal field amplitude, A, must repeat with the same period as the pump pulse train period which is uniquely fixed by the pump laser cavity and other pump laser parameters. If the net gain per pass is not too large, we may smear the gain and loss media to uniformly fill the dye laser cavity. We then seek signal pulses that travel with precisely the same velocity as the pump pulse. There is, however, no reason why these velocities should be equal to the index dependent speed of light, $v$, since the saturable gain and loss of the dye media make it possible for the signal envelope velocity to be somewhat greater than or less than the speed of light.

These concepts may be advantageously used if we introduce a travelling wave temporal coordinate, $\tau=t-z / v_{3}$, where $v_{3}$ is the envelope speed in the dye laser. Formally we also define $\zeta=z$ and seek solutions that are stable with respect to $\zeta$. Since much of the detuning data associated with synchronously pumped laser 
systems are taken in terms of cavity length, it is convenient to recast $v$ and $v_{S}$ in terms of the cavity length of the pump laser, $\mathrm{L}_{\text {pump }}$, and the cavity length of the dye laser, $\mathrm{L}_{\mathrm{dye}}$. Defining the length difference between the two as $\Delta \mathrm{L}=\mathrm{L}_{\text {dye }}-\mathrm{L}_{\text {pump }}$, and incorporating the scattering loss, $\gamma_{S}$, and finite mirror reflectivities into a cold cavity lifetime, $t_{c}$ our set of partial differential equations (126) - (131) reduce to a set of ordinary differential equations in $\tau$ :

$$
\begin{aligned}
& d D / d \tau=-\left(1 / \tau_{2}\right)\left(\left(1+\tau_{2} / 2 \tau_{1}\right) D+\left(1-\tau_{2} / 2 \tau_{1}\right) M+2 Q A x-P(\tau) x^{2}\right] \\
& d M / d \tau=-\left(1 / \tau_{2}\right)\left[-\left(\tau_{2} / 2 \tau_{1}\right) D+\left(\tau_{2} / 2 \tau_{1}\right) M-P(\tau) x^{2}\right] \\
& d Q / d \tau=-\left(1 / T_{S}\right)[Q-A D x] \\
& d A / d \tau=-\left(L / 2 t_{C} \Delta L\right)\left[A-\int_{0}^{1} Q x d x-\int_{0}^{1} Q_{a} y d y\right] \\
& d D_{a} / d \tau=-\left(1 / \tau_{a 2}\right)\left[D_{a}+\alpha+2 B Q_{a} A y\right] \\
& d Q_{a} / d \tau=-\left(1 / T_{a}\right)\left[Q_{a}-A D_{a} y\right]
\end{aligned}
$$

The rate constant, $L / 2 t_{c} \Delta L$, in equation (135) shows the direct relationship between the detuning and the cavity lifetime. Consequently, it is apparent that some control over the cavity matching requirement may be gained by controlling the small signal losses. In particular, the cavity stabilization requirements 
may be made less severe by decreasing $t_{c}$ and this may explain the improved stability reported in references [51] and [62].

Decreasing $t_{c}$, however, also raises the threshold for lasing, and we will see below that this results in longer output pulses of lower intensity. Operating closer to threshold also increases the effects of spontaneous emission.

Equations (132) - (137) constitute a fairly general set that, as we will see below, reasonably models the operation of a hybridly mode-locked dye laser. As the last step in this derivation, we need to assign numerical values to the parameters present in these equations. This task is done next.

\section{PARAMETER VALUES}

The data that we will present below is taken from experiments performed on a Quantronix dual jet hybridly mode-locked dye laser. Used in these experiments was the gain dye, rhodamine $6 G$ and the saturable absorber dye DODCI. In this section we give the parameters pertinent to this laser and also specify a specific pump pulse form. It is important to realize that all the values used in these calculations are the most reasonable values independently gleaned from the literature.

The flourescence decay time of rhodamine $6 G$ is well known and, as in other treatments, a value of $\tau_{2}=5 \times 10^{-9} \mathrm{sec}$. is employed here [24]. The vibrational relaxation time is much shorter and not precisely known. Some studies have indicated that the vibrational relaxation times of both the the upper and lower electronic states of rhodamine 66 are about $(3 \pm 2) \times 10^{-12}$ $\sec [63,64]$. Other research has shown that the vibrational 
relaxation time may be somewhat shorter (at least in the upper electronic state, $\left.S_{1}\right)$ and a relaxation time of $(0.8 \pm 0.2) \times 10^{-12} \mathrm{sec}$. has been deduced [65]. For the present study the value of $\tau_{1}=1 \times$ $10^{-12} \mathrm{sec}$ has been used [24]. The coherence time $T_{\mathrm{s}}$ for rhodamine $6 G$ is shorter still, and its value is even more tenuous. The coherence times for several dyes, including rhodamine $6 G$, have all been found to lie in the range of $0.02 \times 10^{-12} \mathrm{sec}$. to $2 \times 10^{-12} \mathrm{sec}$, depending on solvents, spectral positions, and sample deterioration [66]. A lower bound on the coherence time for rhodamine $6 G$ can be obtained from measurements of the time-resolved flourescence spectrum, and such measurements have shown that immediately after a short pump pulse, the flourescence is a Lorentzian function having a full width at half maximum of $12 \mathrm{~nm}$ [67]. With pure homogeneous broadening, this would correspond to a homogeneous linewidth of $\Delta v_{\mathrm{h}}=1.15 \times 10^{13} \mathrm{~Hz}$. and a minimum coherence time of $2.8 \times 10^{-14} \mathrm{sec}$. Given the limitations of the available data, it was decided here to simply adopt the previously inferred value of $T_{S}=50 \times 10^{-15} \mathrm{sec}$. $[68,69]$.

Fortunately, the fluorescence decay time of DODCI has been well studied by many groups [70 - 74]. Unfortunately, interest in this parameter was driven largely by a disagreement in its value. At moderate excitation levels, a lifetime of approximately $1.2 \times$ $10^{-9} \mathrm{sec}$. has been reported by several independent groups $[70,71,72]$. Under different experimental conditions, a spontaneous lifetime of approximately $0.3 \times 10^{-9} \mathrm{sec}$ has been reported, and it was postulated that this shorter figure is due to a photoisomer formed by the high pump intensity [73,74]. Given the operating conditions of a hybridly mode-locked dye laser, we have chosen to 
use $\tau_{\mathrm{a} 2}=1.2 \times 10^{-9} \mathrm{sec}$, and we find that this value gives good agreement with experiment. Following the measurements in reference [75] and with consideration of the coherence time caveats listed above, we take $\mathrm{T}_{\mathrm{a}}=50 \times 10^{-15} \mathrm{sec}$ for $\mathrm{DODCl}$.

In describing the saturable absorber DQOCI values of $\tau_{a 2}=3 \times$ $10^{-12} \sec [76]$ and $T_{a}=400 \times 10^{-15} \mathrm{sec} .[66,75]$ were used.

Typical cavity lengths for these lasers run between 1.5 and 2 $\mathrm{m}$., and in many of our calculations, we have used $\mathrm{L}=1.8 \mathrm{~m}$. We note, however, that our governing equations may be recast to be explicitly independent of specific cavity length. Typical cavity detunings are in the micron range, and this was an important parameter to vary both experimentally and theoretically. Indeed our verification rests largely on the variation of certain output pulse parameters with $\Delta \mathrm{L}$. In order to estimate the cavity lifetime, we must consider the sum of the losses. The output coupling mirror is usually about $10 \%$ transmitting, but one also needs to estimate the small signal scattering due to two jets, a finite transmission of the tuning filter, etc. With these additional losses in mind, a value of $t_{c}=10$ nsec. was often used in our calculations. The small signal transmission of the absorber jet, $\alpha$, and the saturation ratio, $\beta$, were key parameters of interest, and extensive theoretical mapping with respect to these parameters was done. Reasonable values associated with the Quantronix system are $\alpha=2$, and $\beta=5$.

As a final step in this derivation, it remains to specify a pump pulse form. Following our previous work [24,27] we have chosen the Gaussian shape: 
$P(\tau)=P_{0}(2 / \Delta \tau)(\ln (2) / \pi) \exp \left\{-(2 \tau / \Delta \tau)^{2} \ln (2)\right\}$

$P_{0}$ is a measure of the overall energy in the pump pulse and, for most practical systems is directly proportional to the threshold parameter, $r$, where $r=1$ defines the minimum power needed for lasing. The full width at half maximum (FWHM) of this pump pulse is $\Delta \tau$ and for typical systems in use, $\Delta \tau$ is in the tens of picoseconds. In our modeling of the Quantronix laser, the measured values of $r=1.7$ and $\Delta \tau=35 \mathrm{psec}$. were used. In seneral, these two parameters may be viewed as additional system optimization parameters, and we will detail their effects in a section below.

\section{EXPERIMENTAL RESULTS}

Experiments designed to test our theory were carried out on a hybridly mode-locked rhodamine $6 G$ dye laser with the saturable absorber, DODCI. This is a common, well-characterized combination of dyes. This system is commercially available from Quantronix and represents the latest technology in hybridly mode-locked lasers. In fact, the study was a collaborative effort with the laser's main designer, Dr. Steven N. Janes, and served to further characterize the system for Quantronix.

The pump source was an acousto-optically mode-locked Nd:YLF laser (Quantronix 4200) and delivered a stable pulse train of $35 \mathrm{psec}$ pulses with a pulse repetition frequency of $76 \mathrm{MHz}$. These 1.054 micron pulses were frequency doubled by second harmonic generation in KTP to produce green pulses at $527 \mathrm{~nm}$ that were 
resonant with the $S_{0}-S_{1}$ transition in rhodamine 6G. The power available for pumping the dye laser was limited by the damage threshold of KTP to an average power of $1.0 \mathrm{~W}$. The pump beam was linearly polarized, which, in general, is important for efficient pumping of dyes.

The cavity of the hybridly mode-locked dye laser was matched to the pulse repetition frequency of the pump laser; the nominal cavity lengths for both the pump laser and the dye laser were $1.97 \mathrm{~m}$. For mechanical stability, the dye laser was mounted on a Super-Invar breadboard. The cavity optical train was a linear arrangement with seperate jets for each dye. Saturation in each dye was controlled by tightly focussing the beam at each dye jet. This was accomplished by placing each jet at the waist defined by two focussing mirrors aligned in a confocal configuration, two mirrors with radius of curvature $R$ seperated by a distance $R$. The radius of curvature of the two gain mirrors was $10 \mathrm{~cm}$, the radius of curvature of the saturable absorber mirrors was $5 \mathrm{~cm}$. The length of the gain jet was 300 microns. The shorter Rayleigh length associated with the $5 \mathrm{~cm}$ radius of curvature dictated a shorter jet length of 50 microns for the saturable absorber. Sapphire nozzles and an ethylene glycol solvent were used to minimize turbulence in the jet which adds low frequency noise to the output of the laser. The concentration of of the rhodamine solution was $10^{-3} \mathrm{M}$, while the molarity of the DODCI solution was $10^{-4} \mathrm{M}$. Included in the cavity was a four prism array designed to compensate for dispersion induced chirp. A chirped pulse is one whose frequency (wavelength) changes with local time (position within the pulse) and therefore represents a pulse that is longer than that allowed by its bandwidth. Also included in the cavity was a pellicle etalon that allowed wavelength tuning around 585 
$\mathrm{nm}$. The $1.0 \mathrm{~W}$ of pump power translated to a threshold parameter of approximately $r=1.7$ (an $r=1.0$ represents the minimum power to achieve lasing). This pump power, together with an output coupling of 58 gave $90 \mathrm{~mW}$ of average output power.

With sensitive adjustment of the dye cavity length relative to the pump cavity length, the output pulses of this laser can be as short as 100 fsec. These ultrashort pulses were measured with a common procedure using an autocorrelator. The change in these autocorrelator measurements as a function of cavity length mismatch served as the basis of comparison of our theory with experiment.

The set of equations (132) - (137) was solved numerically using a Sun workstation computer. A Runge-Kutta routine was employed for the differential equation solver while Simpson's rule was used for the integral over the dipole orientation. The point of comparison of the model and the theory was through the autocorrelation of the intensity, measured as described above. The autocorrelations of the numerical solutions were calculated using the definition:

$$
G(\tau)=\int_{-\infty}^{\infty} I(t) I(t+\tau) d t
$$

In particular, the width of the autocorrelation and the peak value of the autocorrelation were used in the comparison. A plot of the full width at half maximum of the autocorrelation, $t_{a c}$, as a function of the cavity length detuning is shown in Fig. 18. The line represents the numerical solutions, while the triangles were read directly from the experimentally measured autocorrelations. 


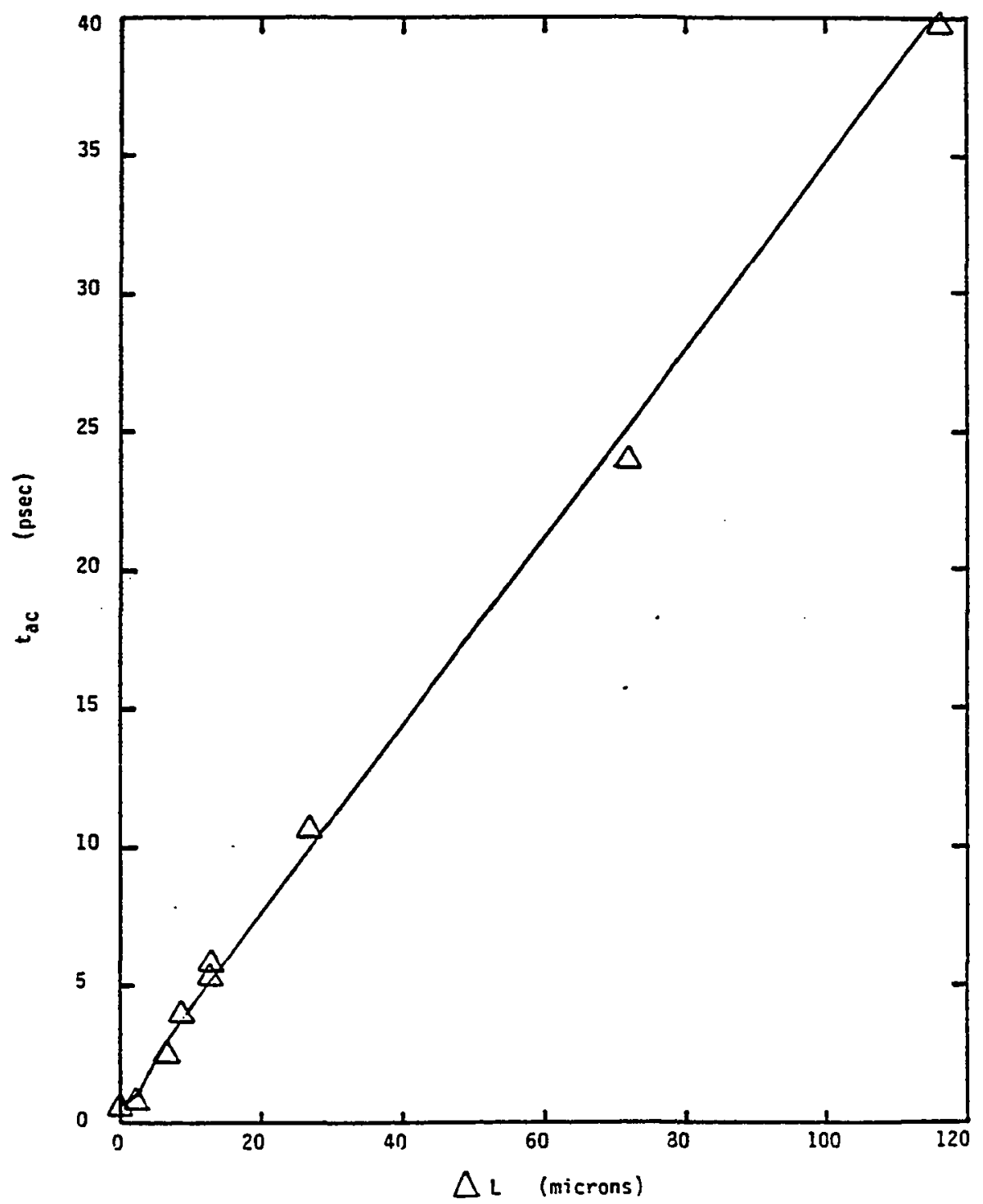

Figure 13. Pulsewidth versus detuning. Hybridly mode-locked dye laser zutocorrelation pulsewith versus cavity 'ength detuning. theory (linej and experument (triangles). 
In Fig. 19 is a plot of the peak magnitude of the autocorrelation, $\mathrm{AC}_{\mathrm{pk}}$. The line corresponds to the numerical solutions while the squares represent the Quantronix data.

The theoretical lines in both cases are in good agreement with experiment.

\section{THEORETICAL RESULTS}

In addition to the time constants associated with the saturable absorber, there are two key parameters that extend the SPML semiclassical mode] of reference [24] to the case of hybrid mode-locking. Specifically, these are the parameters, $\alpha$ and $\beta$. The parameter a represents the small signal transmission or, equivilently, the molarity of the saturable absorber. The parameter $B$ describes the relative saturation of the gain and the absorber and includes first order beam focusing effects. Because, to some extent, $\alpha$ and $\beta$ can be varied experimentally, they are of direct practical interest. This section presents the dependence of the mode-locked pulsewidth, $t_{p}$, and the peak intensity, $I_{p k}$, upon $\alpha$ and $\beta$ as predicted by equations (132) - (138). We concentrate on rhodamine $6 G$ for the gain medium and consider two different saturable absorbers - DODCI and DQOCI. We find that, for reasonable values, the system is only weakly dependent on $\beta$ particularly for the quickly responding dye DQOCI. The numerical value used for these studies included the typical time constants for the dyes as discussed above. In addition the threshold parameter was held constant at $r=3$. A cavity lifetime of $t_{c}=10$ nsec and a cavity length of $1.8 \mathrm{~m}$ were used. A near optimum cavity detuning value of $\Delta \mathrm{L}=10$ microns was used and the pump 


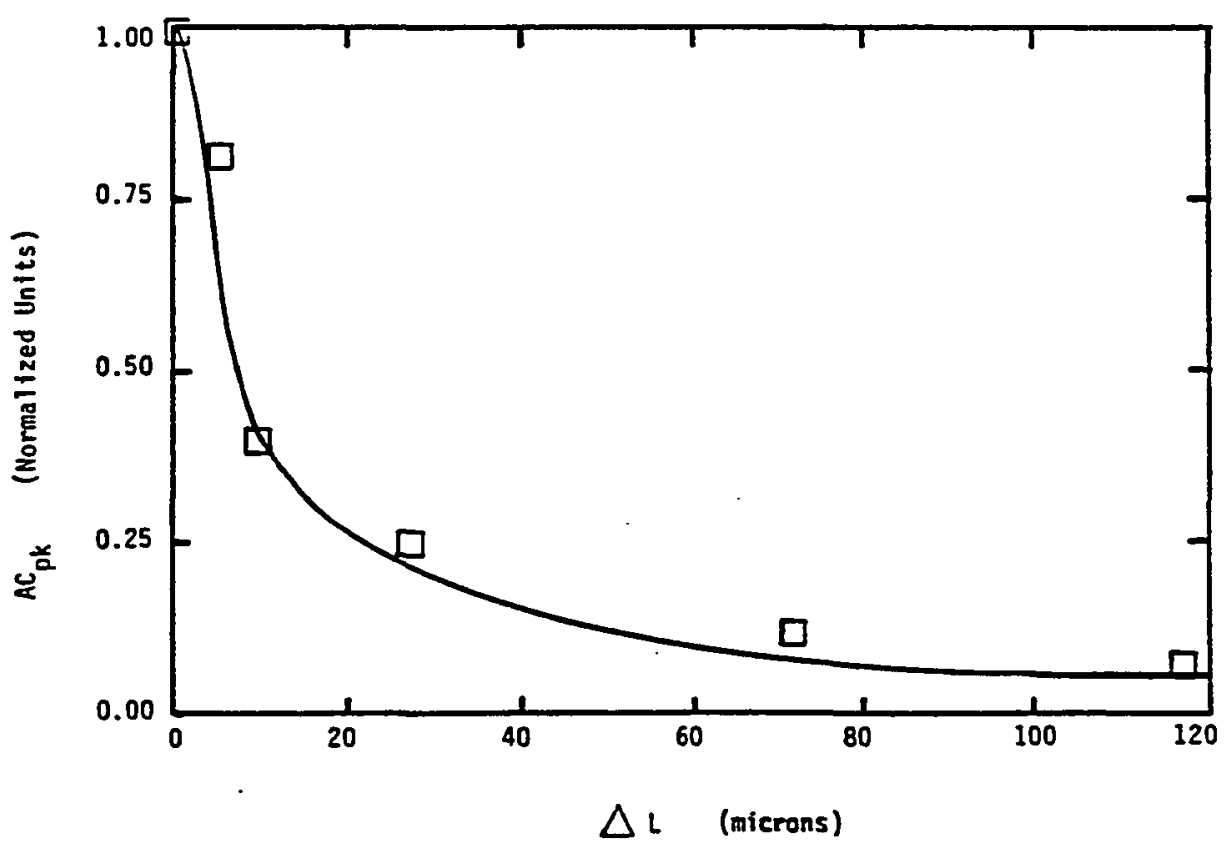

Figure 19. Peak intensity versus detuning. Hybridly mode-locked dye laser autocorrelation peak versus cavity length detuning, theory (line) and experiment (squares). 
pulsewidth was kept at $\Delta \tau=50$ psec.

Figure 20 presents the output pulsewidth as a function of a for the two saturable absorbing dyes. In the semilog plots, there is a marked decrease in pulsewidth as $\alpha$ is increased. This improvement is more pronounced for large $\beta$ for DODCI (Fig. 20a). Interestingly, there is little discernable difference in the output characteristics over three orders of magnitude of $\beta$ for DQOCI (Fig. 20b). The squares on the plots represent approximate regimes of operation of the Quantronix laser system.

In Fig. 21 are similar plots of $I_{p k}$ vs. $\alpha$. There is a marked improvement in the peak intensity at large a's and the improvement is enhanced at large $\beta^{\prime}$ 's. Figure 21a gives the results for DODCI for $\beta=0.10,0.50,1.00$ and 10.0. Figure $21 \mathrm{~b}$ gives the results for $D Q O C l$ for the same $\beta$ 's, however there is no change in $I_{\mathrm{pk}}$ vs $\log (\alpha)$ for this range of $\beta$ 's.

Consequently, pulses that are both shorter and more intense follow from higher absorber molarities and from dyes that have higher saturation parameters. Direct application of this conclusion to experimental practice is complicated by the fact that the minimum power needed for lasing increases as a increases. The plots in Figs. 20 and 21 were calculated at a constant $r$. Thus improvements from increasing $\alpha$ can be realized only if correspondingly more pump power is available. The role of pump power (at fixed $a$ ) is examined more fully below.

The relative insensitivity of the output of the rhodamine $66 / D Q O C I$ system as a function of $\beta$ is an interesting result that may have practical ramifications. It appears from this result that, ceteris paribus, slight decreases in $\beta$ will have no adverse effect on the system's performance. Thus, for the case of DQOCI, one need 
not focus the laser beam as tightly in the absorber jet as for the case of DODCI. This freedom implies less severe optical tolerances and reduced astigmatism.

In the numerical solution of the set (132) - (138) much of the computational time is spent in the formal integration over the dipole orientations in the field equation. The primary issue here, is the quantitative agreement afforded, particularly in the peak intensity, due to the proper treatment of inefficiencies due to dipole misalignment. If one is interested in qualitative study, considerable computational savings arise by considering the specific case of a unidirectional distribution, $n(\theta, \phi)=\mathbf{N} \delta\left(\theta=\theta_{0}, \phi=\right.$ $\phi_{0}$ ), where $\delta$ is a normalized delta function and $\theta_{0}$ and $\phi_{0}$ define the alignment direction. For the particular case of $\theta_{0}=0$ for both $\theta_{\mathrm{s}}$ and $\theta_{a}$, our equations are replaced by:

$$
\begin{aligned}
& \mathrm{dD} / \mathrm{d \tau}_{\tau}=-\left(1 / \tau_{2}\right)\left(\left(1+\tau_{2} / 2 \tau_{1}\right) D+\left(1-\tau_{2} / 2 \tau_{1}\right) M+2 Q A-P(\tau)\right] \\
& d M / d_{\tau}=-\left(1 / \tau_{2}\right)\left[-\left(\tau_{2} / 2 \tau_{1}\right) D+\left(\tau_{2} / 2 \tau_{1}\right) M-P(\tau)\right] \\
& d Q / d \tau=-\left(1 / T_{s}\right)[Q-A D] \\
& d A / d \tau=-\left(L / 2 t_{c} \Delta L\right)\left[A-Q-Q_{a}\right] \\
& d D_{a} / d \tau=-\left(1 / \tau_{a 2}\right)\left[D_{a}+\alpha+2 B Q_{a} A\right] \\
& d Q_{a} / d \tau=-\left(1 / T_{a}\right)\left[Q_{a}-A D_{a}\right]
\end{aligned}
$$


The solution to this set of equations typically underestimates the peak intensity by about $20-30 \%$, but otherwise yields good agreement with the more rigorous solutions.

An important distinction of our governing equations (132) (138) is the inclusion of semiclassical effects through finite coherence times, $T_{s}$ and $T_{a}$. In this section we reduce the unidirectional set $(140)-(145)$ to the rate equation limit and explore the operational range where semiclassical effects significantly manifest themselves. The adiabatic, or quasi-static elimination of the polarization is a well known mathematical technique, and in the limit of $T_{S}=T_{a}=0$, our governing set of rate equations is:

$$
\begin{aligned}
& d D / d \tau=-\left(1 / \tau_{2}\right)\left[\left(1+\tau_{2} / 2 \tau_{1}\right) D+\left(1-\tau_{2} / 2 \tau_{1}\right) M+2 I D-P(\tau)\right] \\
& d M / d \tau=-\left(1 / \tau_{2}\right)\left[-\left(\tau_{2} / 2 \tau_{1}\right) D+\left(\tau_{2} / 2 \tau_{1}\right) M-P(\tau)\right] \\
& d l / d \tau=-\left(L / 2 t_{c} \Delta L\right) I\left[1-D-D_{a}\right] \\
& d D_{a} / d \tau=-\left(1 / \tau_{a 2}\right)\left[D_{a}+a+2 \beta I D_{a}\right]
\end{aligned}
$$

where we have defined the intensity, $I \equiv A^{2}$.

In general, significant distinctions between the solutions to the rate equations, (146) - (149) and the semiclassical equations, (132) (137) arise at small cavity length detunings and at low pump levels. It is important to realize that, experimentally, the shortest output pulses occur at small cavity length detunings and, further, most of today's systems run at modest pump levels. We illustrate 


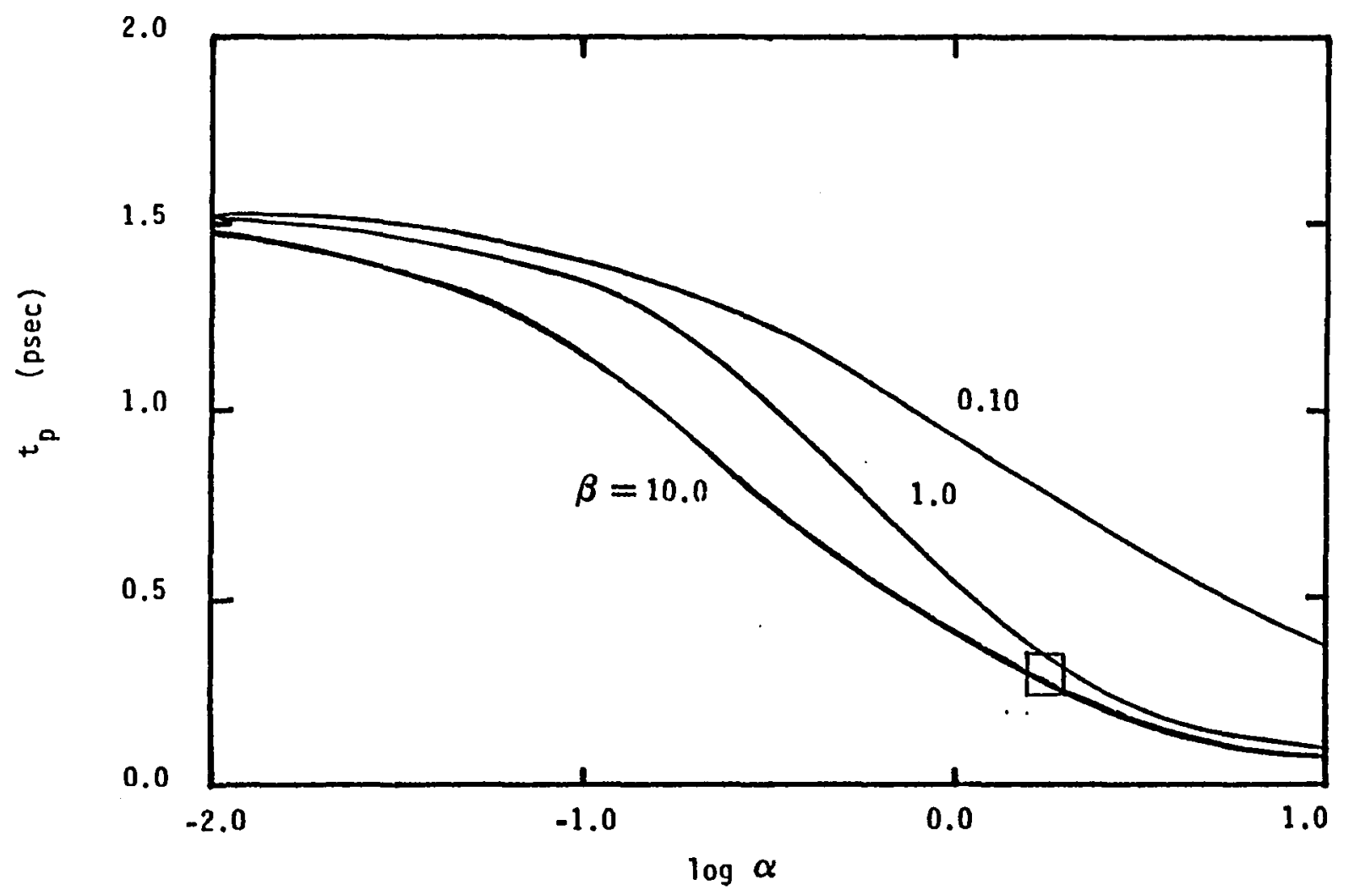

Figure 20a. Theoretical pulsewidth versus DODCI molarity. 


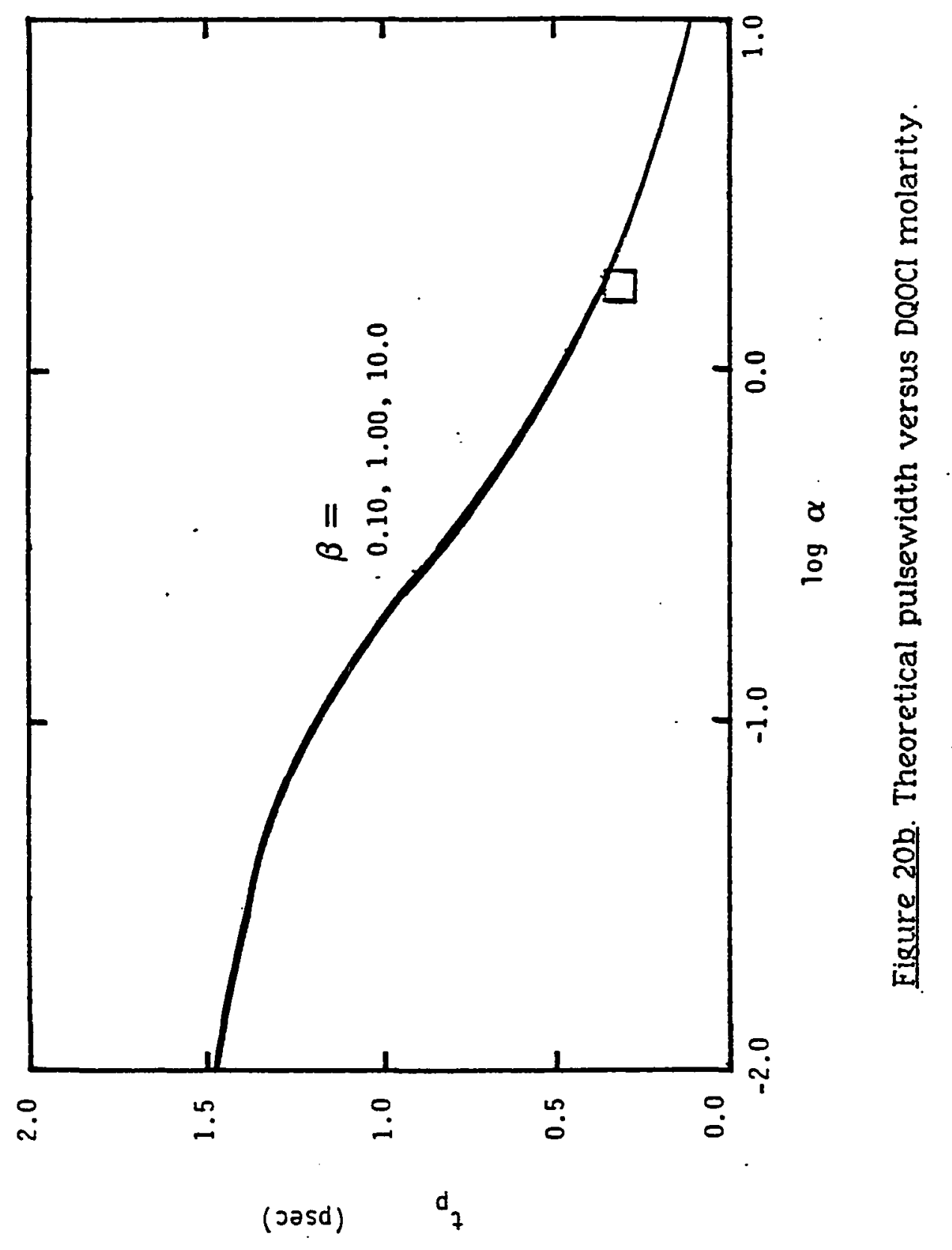




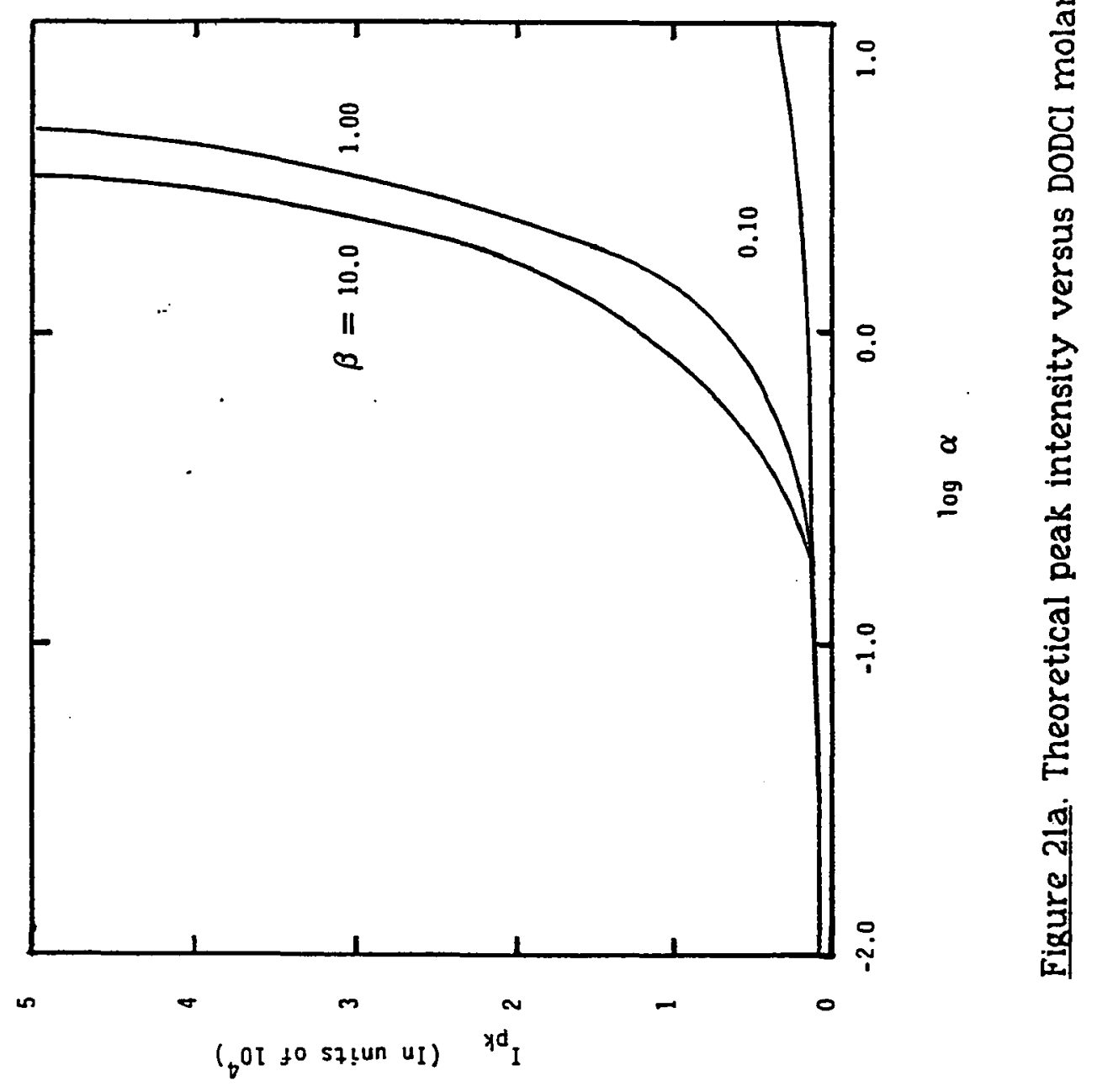




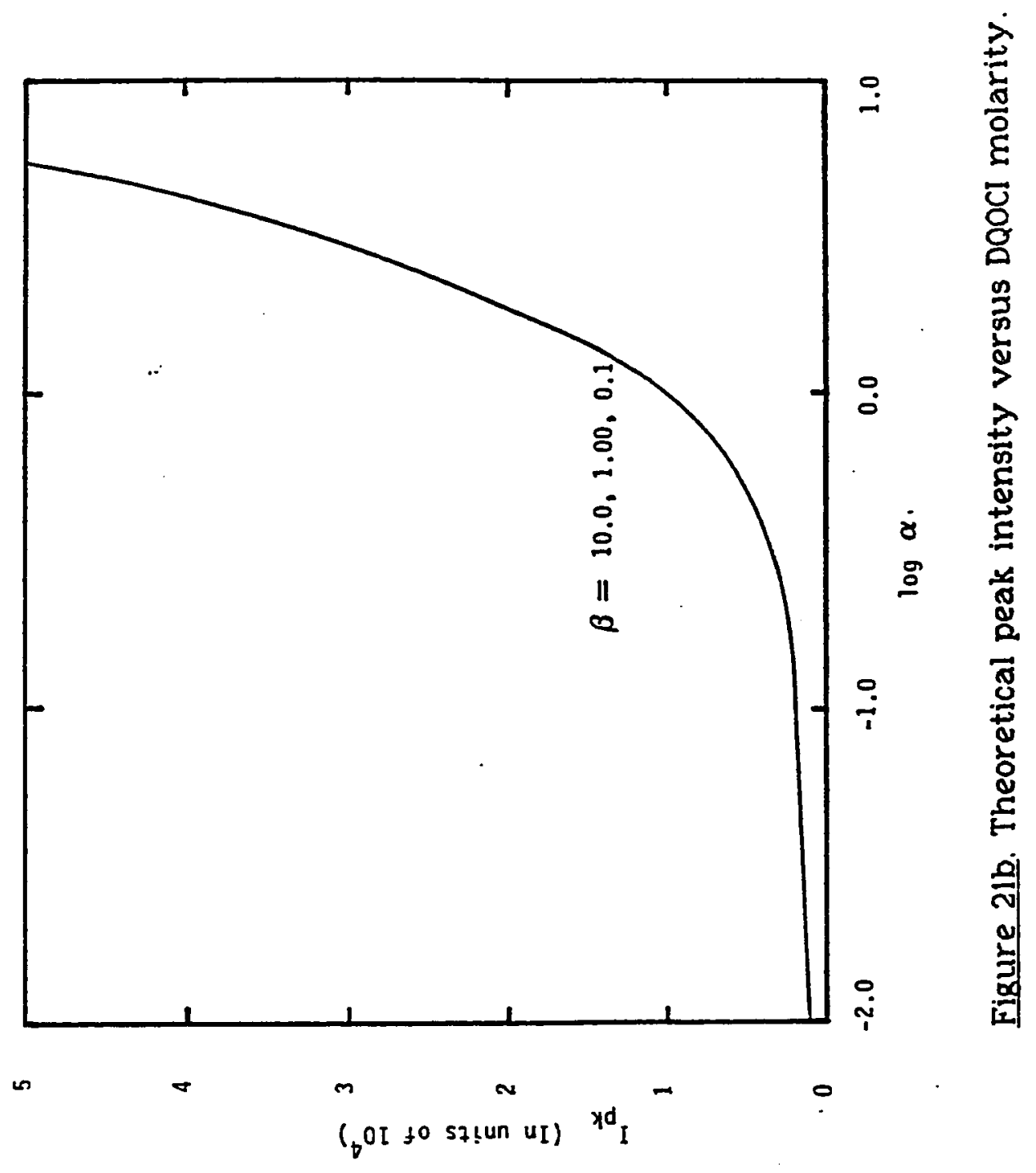


the differences in output pulses in Fig. 22, solutions to the coherent set are plotted with a solid line, while solutions to the simpler, rate equation set are plotted with a dashed line. Pertinent system parameters are as discussed above, with $\Delta \tau=50$ psec, $\alpha=2$ and $\beta=5$. Fig. $22 \mathrm{a}$ is for $r=3.0$ and $\Delta \mathrm{L}=10$ microns. Apart from a shift in when the pulse occurs, there is reasonable agreement in pulse shape and satellite structure. Fig. 22b, however, is for the perhaps more realistic values of $r=1.5$ and $\Delta \mathrm{L}$ $=5$ microns, and predicts pulses that are both more intense and temporally shorter. Here the neglect of semiclassical effects result in significant discrepancies. These conclusions are similar to those found for SPML lasers in reference [24].

It has been suggested that, experimentally speaking, a lack of satellites in the autocorrelation trace is an indication of hybridly mode-locked operation rather than SPML operation $[46,51,54]$. However, we note from Fig 22, that if $r$ is large enough, satellite pulses can readilly appear even with a significant amount of saturable absorber. This is not necessarilly in contradiction with these experimental observations since the increase in absorber molarity which is desirable from the standpoint of short pulses also lowers the effective $r$ (for a given pump power) and it is this, in turn, that inhibits satellite pulses.

In the above sections we have derived and verified a semiclassical model the describes the operation of hybridly mode-locked lasers. Additionally, we have explored the sensitivity of the model to certain important parameters such as absorber molarity, relative saturation and coherence effects. A general goal of theoretical understanding is the ability to predict improvements in performance by identifying critical elements of a given technology. In this section we study the role of the pump pulse in 


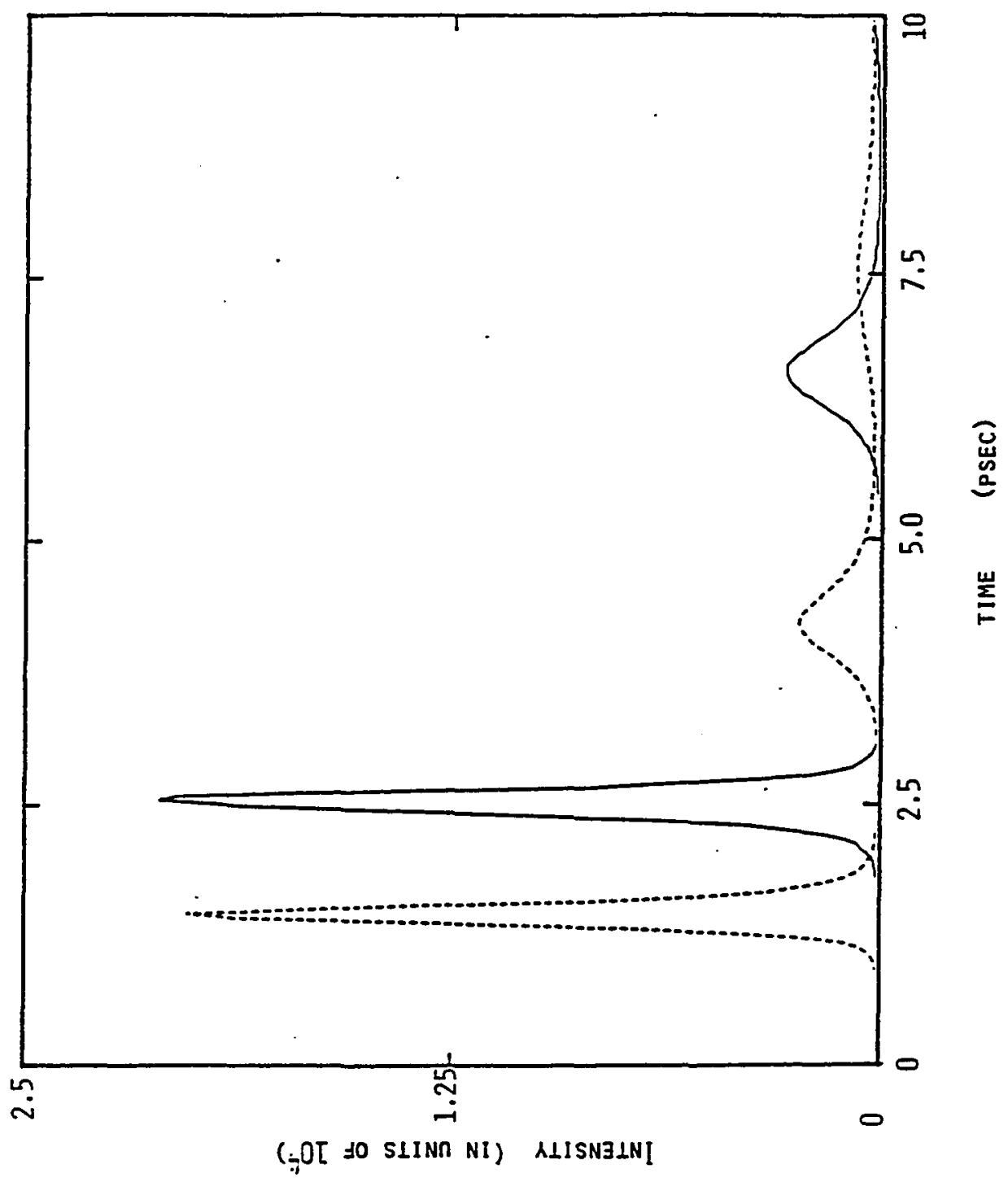

Figure 22a. Thesretical pulselorms. Calculated output pulses including semiciassical effects (solid line) and in the rate equation limit (dashed line) for $r=3.0$ and $\Delta L=10$ microns. 


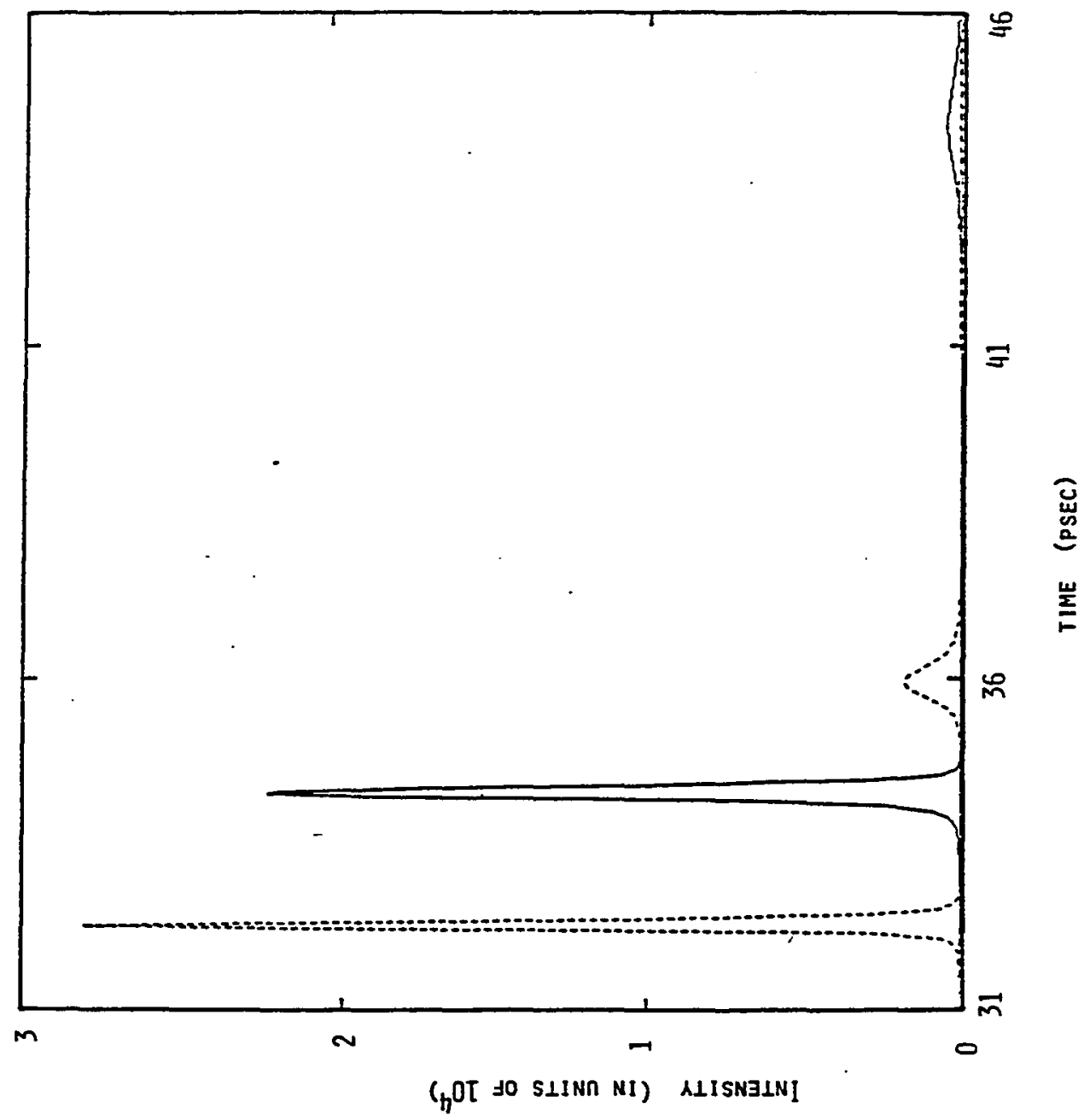

Figure 22b. Theoretical pulseforms. Calculated output pulses including semiclassical effects (solid line) and in the rate equation limit (dashed line) for $\mathrm{T}=1.5$ and $\Delta \mathrm{L}=5$ microns. 
the formation of the ultrashort optical pulses in a hybridly mode-locked laser. Specifically we view the pump pulsewidth and the pump power as optimization parameters that can produce hybridly mode-locked laser pulses that are significantly shorter and of higher peak intensity than those from current systems.

Figure 23a shows the decrease in pulsewidth as $r$ increases for three values of $\Delta \tau$. Most systems today operate at approximately $r=2.0$, and thus we note that some improvement may still be attainable before the point of diminishing returns sets in near $r=$ 5. In Fig. $23 \mathrm{~b}$ is a plot of the peak intensity vs. $r$ for $\Delta \tau=50,10$ and 1 psec. As expected, $\mathrm{l}_{\mathrm{pk}}$ increases as $r$ increases. Further, at high $r$ 's, there is a dramatic improvement in ${ }^{p k}$ when $\Delta \tau$ is shortened from 10 psec to 1 psec.

Figure 24a shows the improvement in pulsewidth to be had as the pump pulse is shortened. We see from the semilog plot that a factor of $2-3$ improvement in pulsewidth may be had if the pump pulsewidth is reduced to below $1 \mathrm{psec}$. Similar improvements in $\mathrm{I}_{\mathrm{pk}}$ would also be achieved with short pump pulses, and Fig. $24 \mathrm{~b}$ shows our calculated $\mathrm{I}_{\mathrm{pk}}$ vs. $\Delta \tau$ dependence. Noteworthy in Figs $24 \mathrm{a}$ and $24 \mathrm{~b}$ is that neither $t_{\mathrm{p}}$ nor $\mathrm{I}_{\mathrm{pk}}$ changes beyond $\Delta \tau=1 \mathrm{psec}$. for any $\mathrm{r}$. Further, no other parameter shape, formation time, etc - undergoes a measurable change below $\Delta \tau=1$ psec. We conclude, then, that this is the Dirac delta function limit for the rhodamine $6 G$ : DODCI hybridly mode-locked dye laser system. From a practical standpoint, this regime represents the optimum operating point for these lasers.

Consequently, current hybridly mode-locked laser systems may be further optimized with respect to the pump pulse. We note that this source of improvement is not available in 
conventional colliding pulse mode-locked lasers which are excited by a cw pump. We calculate that the onset of the delta function limit, the regime where the shortest output pulses occur, begins when the pump pulses are approximately $1 \mathrm{psec}$ in duration. To date there are no reports of a hybridly mode-locked laser pumped with this short a pulse. However, this limit has been demonstrated in a variety of SPML dye lasers, and we may speculate on the application of these techniques to hybridly mode-locked lasers. One method of producing pump pulses as short as 1 psec might involve using a SPML dye laser to pump the hybridly mode-locked laser. A subpicosecond SPML dye laser pumped SPML dye laser was demonstrated by Heritage and Jain in 1978 [26], however no saturable absorber was included in that three laser system. More recently have been the successes of Johnson and Simpson [7] and others [8,77] in using fiber/grating compressed, frequency doubled Nd:YAG lasers to pump SPML dye lasers. These systems produce the shortest pulses - approximately 200 fsec - reported from a SPML dye laser. Consequently it appears that suitable picosecond pump pulses are attainable with current technology.

\section{CONCLUSIONS}

In this chapter we have presented the detailed derivation of a theory that governs the operation of a hybridly mode-locked dye laser. This model was verified through comparison with experiments performed on a Quantronix Nd:YLF pumped, rhodamine 6G/DODCI, hybridly mode-locked dye laser. Good agreement between experiment and theory was found in the output pulsewidths and peak intensities over a wide range of 
cavity length detunings.

With this agreement as a starting point, we have explored our model numerically. In particular, we have studied the role of the saturable absorber with regard to absorber molarity and relative saturation. From our earlier work with SPML lasers $[24,27]$ we can conclude that the role of the saturable absorber is primarily one of shaping and shortening the mode-locked pulse.

This nonlinear dynamical model includes such features as semiclassical and dipole orientational effects, and we have found that both of these phenomena must be included in order to quantitatively model hybridly mode-locked dye lasers.

We have also used this model to explore possible improvements in output pulse width and intensity through optimization of the pump pulse. We predict that existing hybridly mode-locked dye lasers would see a 2- to 3-fold shortening in output pulse widths and a 3- to 5-fold increase in peak intensity if 1 picosecond pump pulses were used.

We have presented a nonlinear dynamical model that makes no prior assumptions about pulse presence or shape. This allows us to interpret hybridly mode-locked laser operation as the transient solution to a set of coupled Maxwell-Schrodinger equations. This has practical ramifications; our model predicts a pulse shape and therefore allows more detailed interpretation of measured autocorrelations. In spite of the good agreement with experiment, we feel that further work in this area is needed in the inclusion of detuning from line center frequencies, inhomogeneous broadening, bandwidth limiting tuning elements, intracavity dispersion compensation and pulse train instabilities. Our formalism can support studies of these considerations at the expense of additional computing time. 


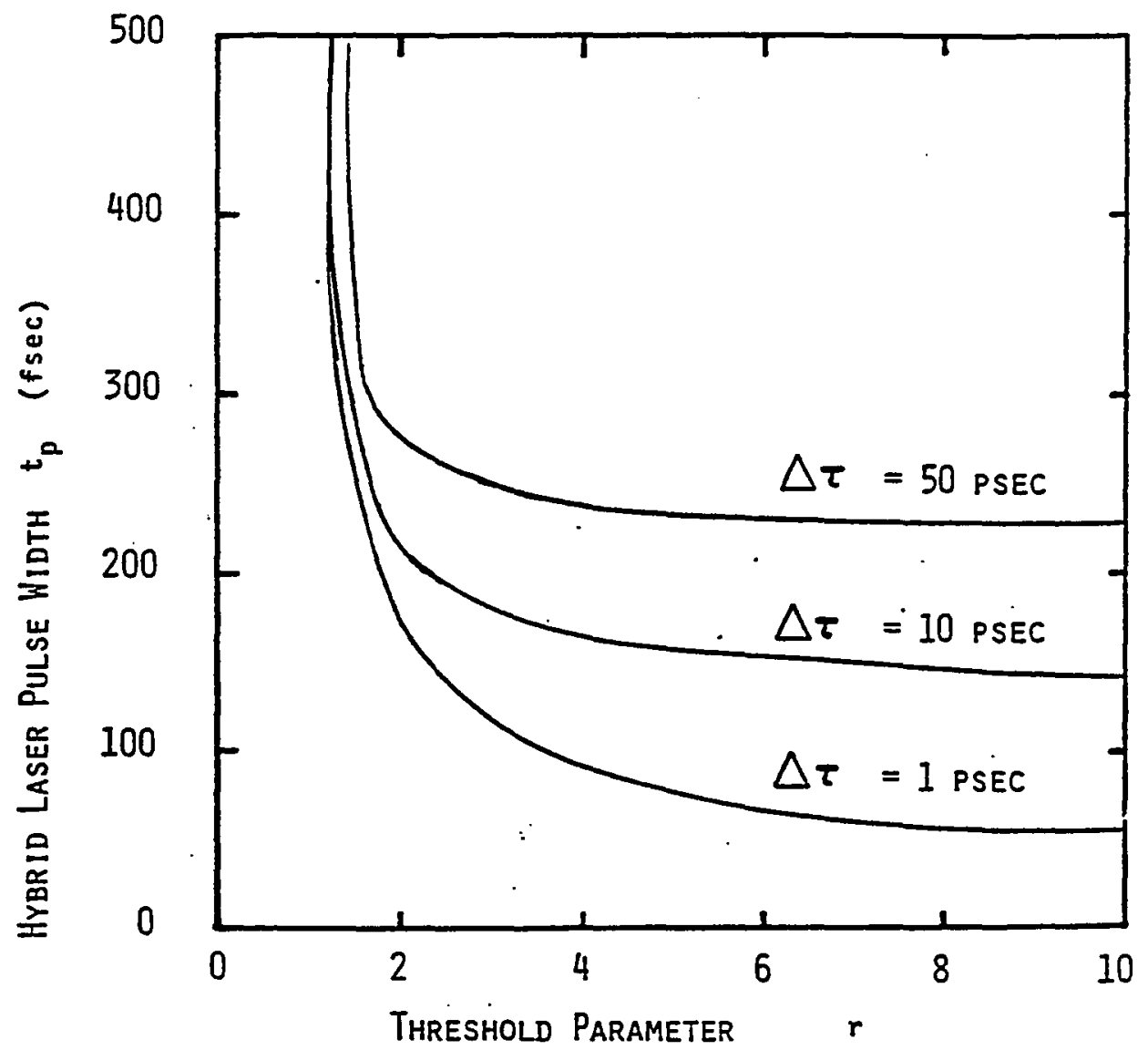

Figure 23 a. Theoretical pulsewidth versus $r$. . 


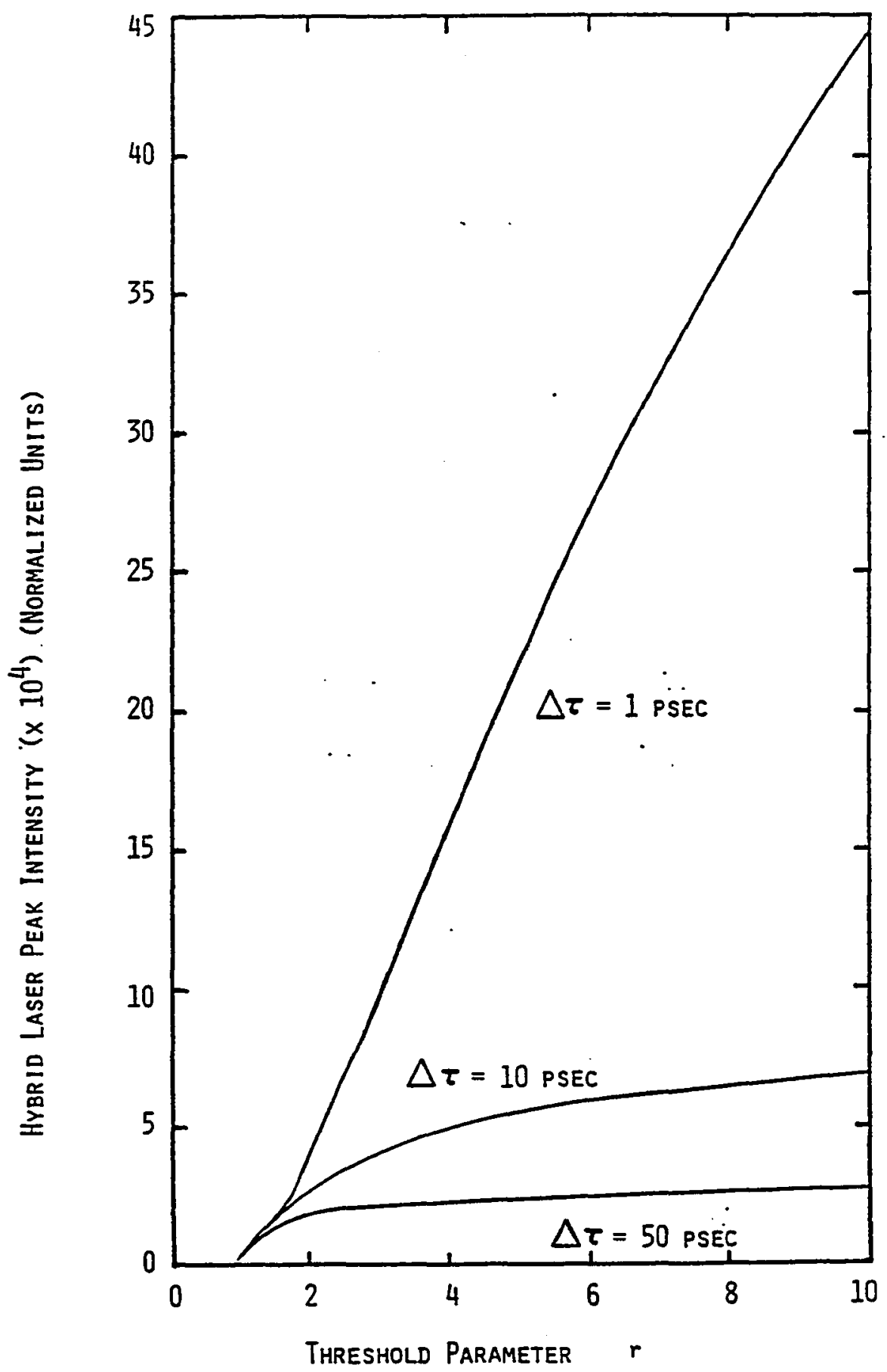

Elsure 23b. Theoretical peak intensity versus $r$. 


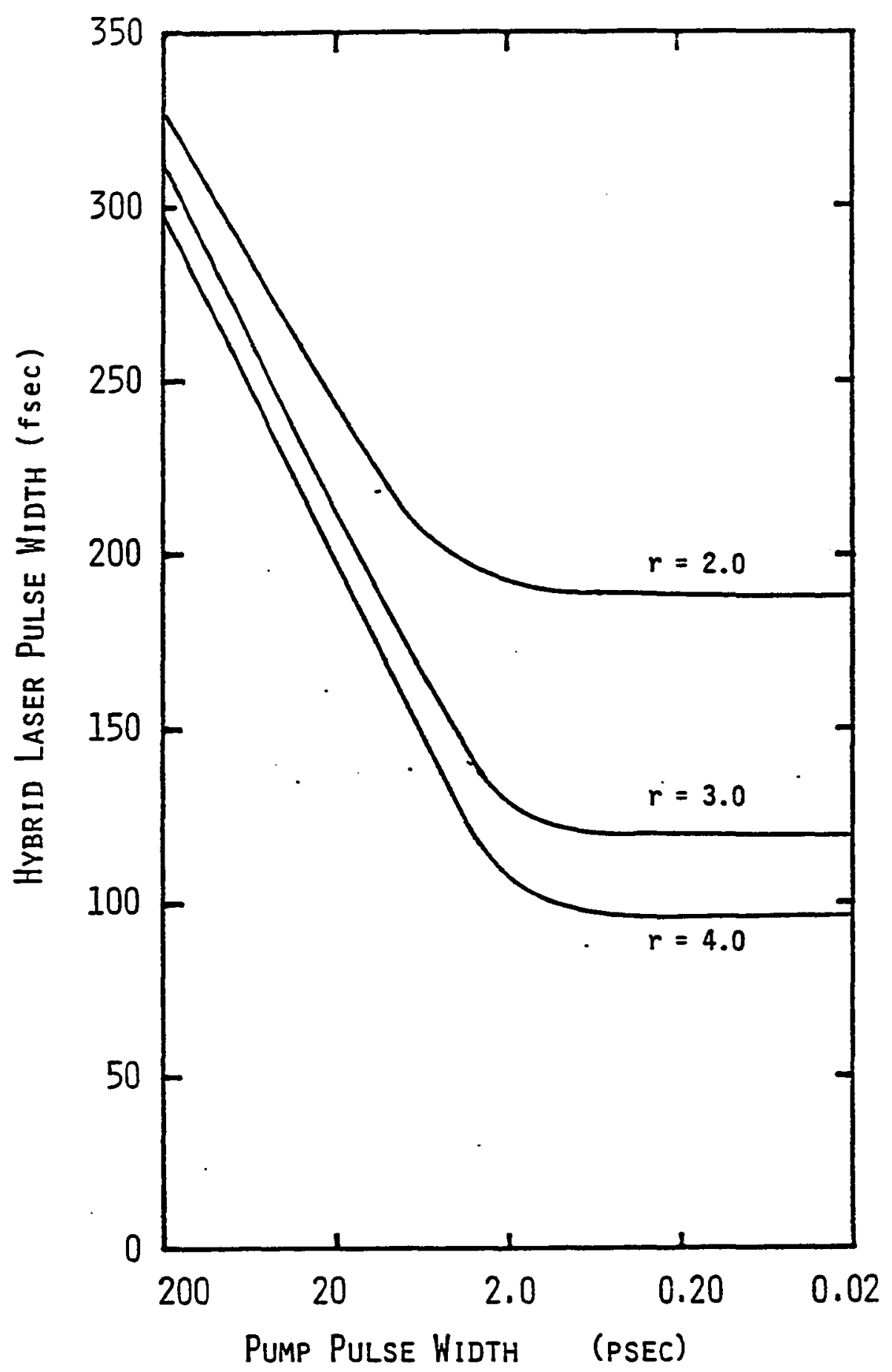

Figure 24a. Theoretical pulsewidth versus pump pulsewidth 


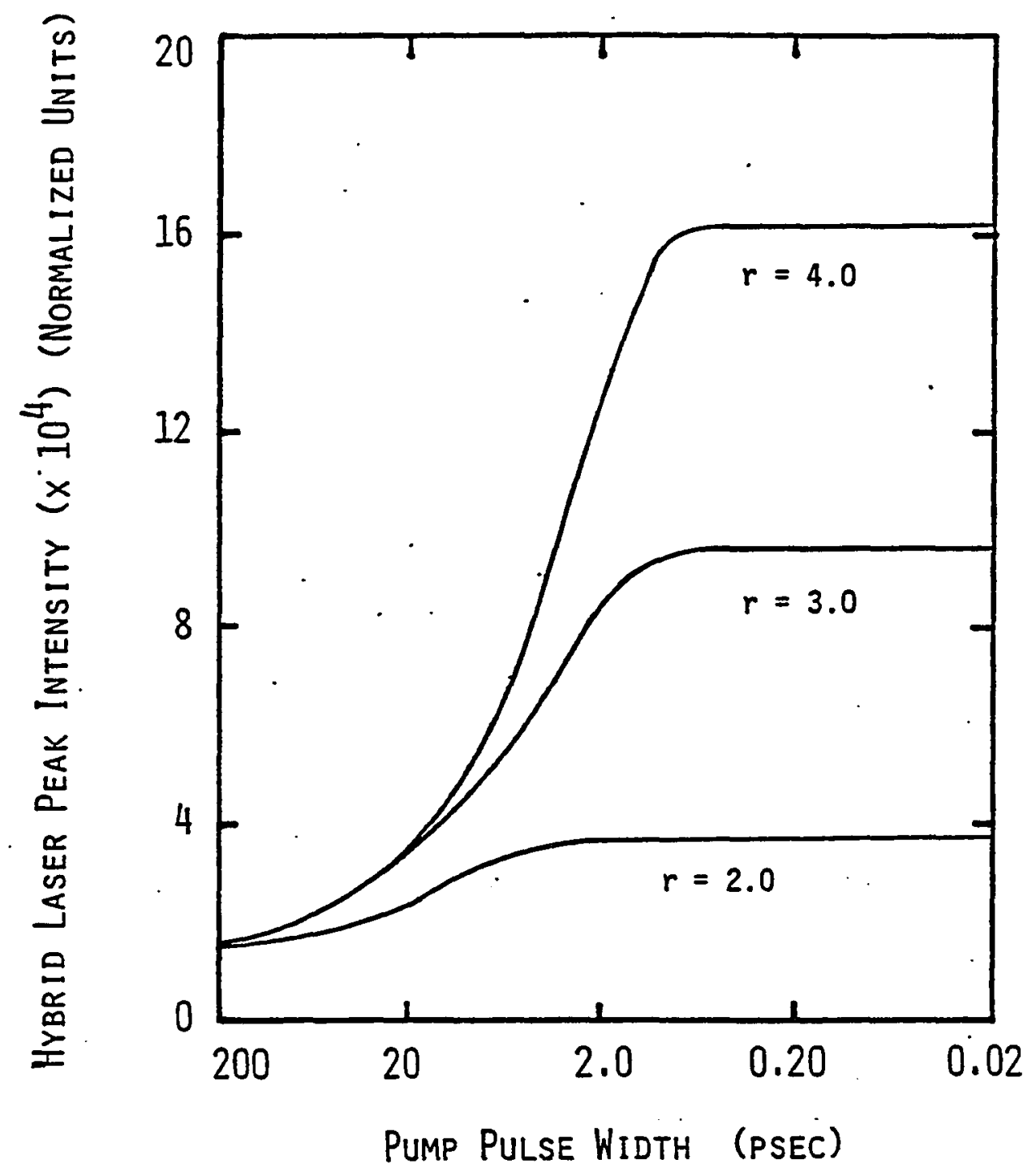

Figure 24b. Theoretical peak intensity versus pump pulsewidth. 


\section{CHAPTER V}

\section{SPECTRAL BEHAVIOR AND PULSE TRAIN INSTABILITIES OF A SYNCHRONOUSLY PUMPED MODE-LOCKED DYE LASER}

\section{INTRODUCTION}

Since the first reports of active mode-locking in lasers [78], much demand has arisen for the stable, ultrashort optical pulses produced by this technique. Consequently, much effort has gone into the theory and practical realization of reliable mode-locked pulse trains that are pulse-to-pulse consistent. A class of considerable interest is the synchronously pumped mode-locked (SPML) dye laser which currently finds wide use as a tunable source of picosecond and even subpicosecond optical pulses [72]. A typical system employs a cw mode-locked pump laser such as an acousto-optically mode-locked argon ion laser whose cavity length is almost equal to the cavity length of the dye laser. Since the earliest experimental reports $[2,4]$ this arrangement has been refined into a turnkey commercial system.

The earliest theoretical studies of SPML lasers used a rate equation analysis to study steady state pulse parameters as functions of the length mismatch between the pump laser cavity and that of the dye laser $[14,15]$. Later papers augmented these treatments, but it is only recently that theories have questioned the steady state or self-consistent pulse profile approach $[16,57,80,81]$. Experimentally, Kluge et al. have studied the 
subpicosecond temporal jitter on the output train of a SPML dye laser, that they attribute to the jitter of the pump laser [82]. The theoretical studies of New and Catherall, however, have suggested that a SPML laser will have a fundamental source of jitter [80]. Further, streak camera studies have suggested that there are cavity length regimes - particularly where the shortest mode-locked pulses occur - where SPML dye lasers are unstable [83]. Separately, Baer and Smith have reported on the "comb structured" rf spectra of a slightly mismatched SPML dye laser [84].

This chapter presents the results of a systematic experimental study of the effect of small cavity detunings on the pulse train envelope of a SPML rhodamine $6 G$ dye laser. While the behavior of the laser is rich and varied, of practical interest is the observation of a slow, erratic amplitude modulation at the cavity length that produces the shortest pulses when the bandwidth limiting tuning filter is included in the dye laser cavity. Since most theories governing SPML dye lasers employ some form of a self-consistent pulse approximation, the behavior reported herein has not been predicted. However, significant variations (up to 208) occur only over the course of hundreds of pulses. Thus, actual pulse-to-pulse variations are small, and the self-consistent pulse approximation remains reasonable for many purposes.

Zheng et al. have reported a sequence of period doubling bifurcations associated with extreme (30\%) length mismatches between a mode-locked argon ion pump laser and a rhodamine dye laser and suggest that this system is appropriate for studies of optical chaos [85]. The range of cavity detunings explored here, however, is kept to within one part in $10^{3}$ - a physical length shift of $1.4 \mathrm{~mm}$. This detuning range and the observed $\sim 10^{5} \mathrm{~Hz}$ 
amplitude modulation is parallel to a recent study of an acousto-optically mode-locked argon ion laser [86]. Reference [86] (which may be found in the appendix) and this exploration together suggest that a slow erratic envelope modulation may be a general characteristic of slightly detuned actively mode-locked laser systems. An understanding of this instability may lead to a more thoughtful design of mode-locking drivers.

Similar time domain studies have also been carried out without the filter in the cavity. Interestingly, no erratic behavior of the pulse train envelope is observed when this linear element is omitted. However, a slow (microsecond) periodic modulation of the pulse train is identically present with and without the filter.

Unlike the periodic modulation reported in [86] there is no onset of erratic behavior upon further detuning. For the dye laser there is only a linear increase in fundamental modulation frequency with detuning.

There has recently been considerable interest in the bichromatic spectra emitted by $\mathrm{cw}$ dye lasers at suitable values of the threshold parameter, $r[87,88]$. In this study we also examine the spectroscopy of an SPML dye laser as a function of the cavity length detuning, and certain types of multiwavelength emissions are reported here.

\section{EXPERIMENTAL SETUP}

The experiments were performed using a commercially available Spectra-Physics argon ion/rhodamine $6 G$ dye laser system with cavity lengths of $186 \mathrm{~cm}$. The argon laser (Spectra-Physics 2020) was actively mode-locked by a fused silica 
acousto-optic modulator driven at about $40.25 \mathrm{MHz}$. The argon

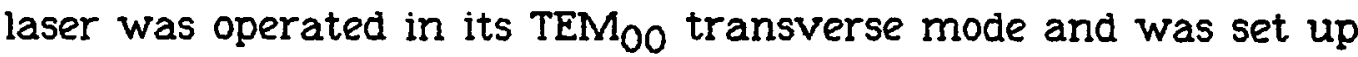
to produce a stable train of $100 \mathrm{psec}, 514 \mathrm{~nm}$ pulses with a pulse repetition frequency (PRF) of $80.5 \mathrm{MHz}$. Any jitter on this pulse train was more than $60 \mathrm{db}$ below the level of the 80.5 PRF component.

The argon laser pumped a matched cavity length Spectra-Physics 375B dye laser at a level approximately 2.4 times above threshold. The details of this arrangement are presented in Fig. 25. The $2 \times 10^{-3}$ molar (rhodamine 66 in ethylene glycol) dye jet was set at Brewster's angle to the argon laser beam. The output coupler was mounted on a translatable stage which provided the cavity length adjustment. A Mitutoyo dial indicator allowed displacement measurements readable to $0.5 \mu \mathrm{m}$ resolution and the relative system length error was less than $\pm 2 \mu \mathrm{m}$. For our purposes the optimum cavity length was defined as that giving the shortest mode-locked pulse as measured by a Spectra-Physics 409 autocorrelator. Length detunings were then measured as displacements away from this position. A reasonable estimate of this optimum difference between the length of the argon laser and the dye laser can be infered from reference [24] and for this case is approximately $50 \mu \mathrm{m}$.

The temporal character of the pulse train was studied using two fast Si PIN diodes (Antronics S-2) operating photoconductively. The entire cross section of the laser beam was focused onto the detectors, although no variations in the effects reported here were observed when the detector was scanned radially across the beam. Even though the $35 \mathrm{psec}$ risetime of these detectors prevented any study of individual pulse shape, their signals provided an adequate measure of the total energy in each pulse. One of the signals was 
fed to a Tektronix 7112 rf spectrum analyzer in a 7904 mainframe. This setup allowed constant monitoring of the homodyne spectrum as a function of length detuning. In general, it was convenient to focus on the frequency range of $\pm 1 \mathrm{MHz}$ around the $80.5 \mathrm{MHz}, \mathrm{c} / 2 \mathrm{~L}$, pulse repetition frequency. The second photodiode was used to study the envelope in the time domain using a Tektronix 7834 storage scope. The storage mode was essential in viewing some of the irregular (non-triggerable) signals. Also monitored was the average output power as measured by a Coherent 212 power meter. Interestingly, the average power remained fairly constant at approximately $75 \mathrm{~mW}( \pm 108)$ throughout the range of detunings.

Generally included in the cavity was a two plate birefringent filter with a measured bandwidth of $12 \mathrm{~nm}$ (FWHM). This element allowed 2 psec pulses that were tunable across the rhodamine gain spectrum. When the birefringent filter was removed, the operating wavelength and the bandwidth of the dye laser were no longer predetermined and the resulting spectroscopy of the laser became rich and varied. The wavelength dependence of the average power was studied using a motor driven $1 / 2$-meter Jarrell-Ash monochromator. A Coherent 212 power meter measured the average power transmitted by the monochromator. This analog signal was recorded on a Hewlett Packard 7047 A $x-y$ recorder. 


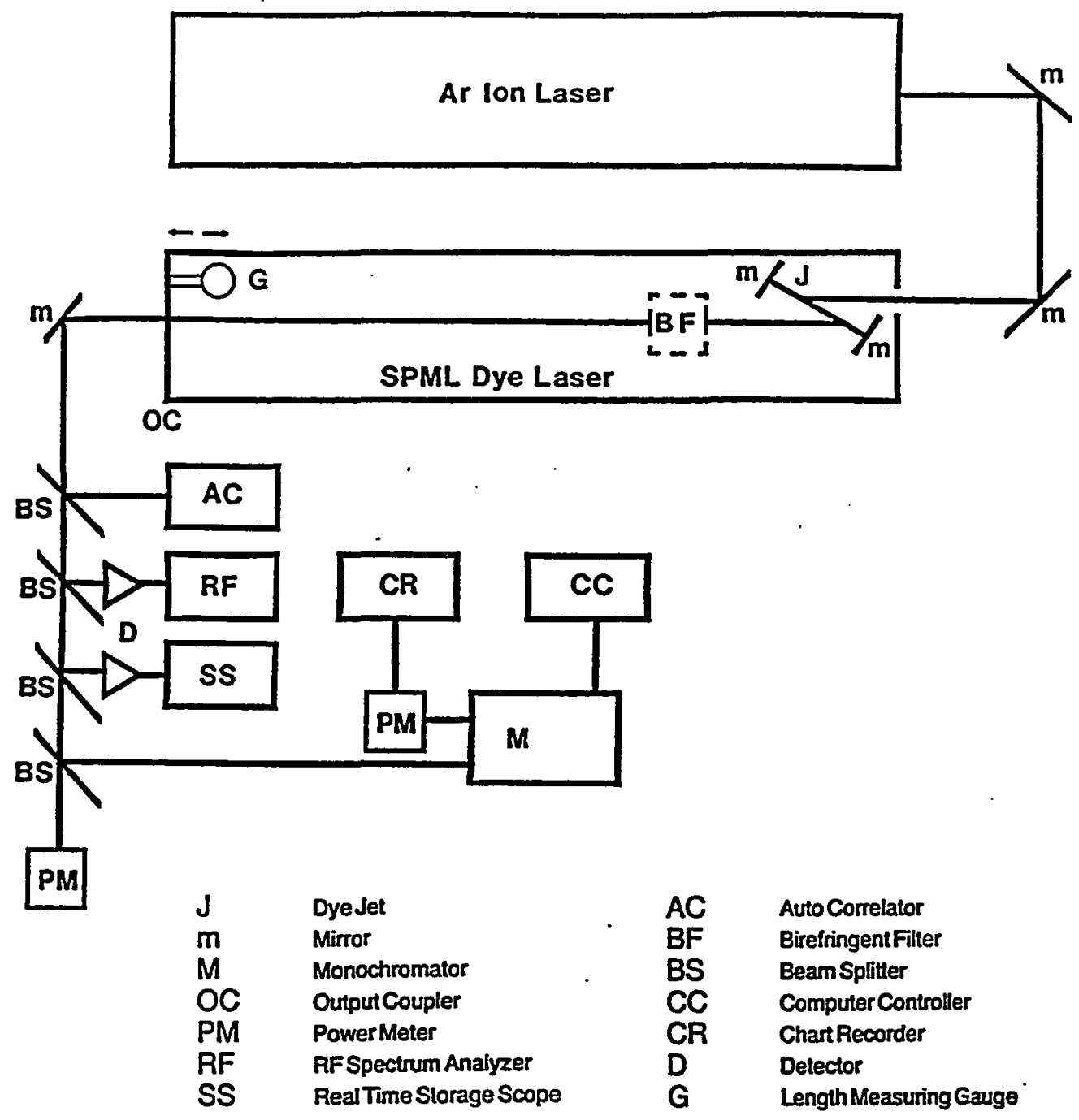

Fiqure 25. Experimental setup. 
Of immediate practical interest is the observation of a slow erratic modulation on the pulse train at the optimum length detuning with the inclusion of a bandwidth limiting, wavelength tuning birefringent filter. Much of the data in the following paragraphs have been taken with the birefringent filter in place and tuned to the peak wavelength of $599 \mathrm{~nm}$, however the data is qualitatively similar at all wavelengths across the rhodamine gain curve. Figure 26(a) is a storage scope trace of the pulse train envelope at the optimum cavity length. On the $2 \mu 5 s /$ div scale shown here, the individual pulses are crowded together and the envelope appears as a continuous trace showing significant modulation. The frequency content of this envelope is shown in the rf spectrum analyzer trace of Fig. 26(b). The horizontal scale is $200 \mathrm{kHz} /$ div. The central peak is the $80.5 \mathrm{MHz}, \mathrm{c} / 2 \mathrm{~L}$, pulse repetition frequency and is surrounded by a broad background that lifts off the baseline. This homodyne spectrum reflects an irregular amplitude modulation of the pulse train with frequency components out to $800 \mathrm{kHz}$.

Pulses of under $10 \mathrm{psec}$ duration are produced only if the cavity length is between $L_{\text {opt }}-10 \mu \mathrm{m}$ and $L_{\text {opt }}+30 \mu \mathrm{m}$. The irregular envelope shown in Fig. 26(a) is present throughout this $40 \mu \mathrm{m}$ range of lengths. The modulation depth, however, does decrease as the pulse length increases. At $\mathrm{L}=\mathrm{L}_{\mathrm{opt}}+35 \mu \mathrm{m}$ the modulation is $60 \mathrm{db}$ down, while the pulsewidths are greater than 30 psec. Further detuning to $L=L_{o p t}+92 \mu \mathrm{m}$ produces a deep (258), regular envelope modulation. This clean periodic modulation persists for further length increases until lasing stops at $L=L_{\text {opt }}+$ 
$950 \mu \mathrm{m}$. Figure 27 shows temporal traces and homodyne spectra for $L=L_{o p t}+125 \mu \mathrm{m}$ and $L=L_{o p t}+320 \mu \mathrm{m}$. As the cavity length increases through this regime, the modulation frequency increases linearly with a slope of $1.09 \mathrm{kHz} / \mathrm{\mu m}$ as shown in Fig. 28 . While this periodic modulation sets in with an abrupt threshold at a detuning of $+92 \mu \mathrm{m}$, if one projects a best fit line through a plot of modulation frequency versus detuning, one finds a modulation frequency of zero at a detuning of $+3 \mu \mathrm{m}$ - effectively at the optimum length.

The phenomenology is more varied when $L$ < $L_{\text {opt }}$. The broadband noise present at $\mathrm{L}_{\text {opt }}$ persists as $\mathrm{L}$ is initially shortened. At first the bandwidth of the envelope narrows and the modulation depth gradually decreases. At $\mathrm{L}=\mathrm{L}_{\mathrm{opt}}-60 \mu \mathrm{m}$, there is a sharp transition point where several strong sidebands grow from the background. In the time domain this appears as a sudden increase in the modulation depth. The pulse train envelope and its homodyne spectra at this length are shown in Fig. 29. This modulation diminishes upon further detuning until, at $L=L_{o p t}-$ $80 \mu \mathrm{m}$, the envelope is essentially flat. A second sharp transition into modulation occurs at $\mathrm{L}=\mathrm{L}_{\mathrm{opt}}-123 \mu \mathrm{m}$ where a strong, noisy modulation develops, as shown in Fig. 30. The irregularity in this signal disappears and the modulation depth grows during the next $5 \mu \mathrm{m}$ length decrease. This case is shown in Fig. 31 for $\mathrm{L}=\mathrm{L}_{\mathrm{opt}}$ 128. The power spectrum of Fig. 31(b) suggests that a period 5 bifurcation has occured since $L=L_{\text {opt }}-123 \mu \mathrm{m}$. Over the next 100 $\mu \mathrm{m}$ these smaller components grow and frequency components fill in between them. In the time domain there is an irregular 
envelope with a $50 \%$ modulation depth. Lasing stops at $I=L_{\text {opt }}-$ $280 \mu \mathrm{m}$.

The data described above was taken with the birefringent filter in the dye laser cavity. The pulse train phenomenology is markedly simpler when this element is removed. In particular, there is no length regime where the envelope of the pulse train may be called erratic, including right about $L_{\text {opt }}$. While there are areas for $L<L_{\text {opt }}$ where there is a measurable background on the rf spectrum, its level is always down by more than $60 \mathrm{db}$ from the 80.5 PRF component.

A characteristic that identically occurs whether or not the tuning filter is in the cavity, however, is the clean periodic envelope modulation as in Fig. 27. Temporal data taken without the filter gives the same sudden onset at $\mathrm{L}_{\mathrm{opt}}+92 \mu \mathrm{m}$, and the same linear shift in frequency upon further detuning as shown in Fig. 28.

SPECTRAL BEHAVIOR OF A FREE RUNNING SPML DYE LASER

When the dye laser is free running, that is to say, when there is no wavelength determining intracavity filter, the peak lasing wavelength, the bandwidth and the shape of the spectra all become functions of the cavity length. The fact that the lasing wavelength changes with cavity length is not an anticipated result and does not occur in cW dye lasers. In Fig. 32 is a plot of lasing wavelength vs. cavity detuning. Immediately evident are striking transitions in $\lambda_{\text {peak }}$ at $\Delta \mathrm{L}=-437 \mu \mathrm{m}$ and $\Delta \mathrm{L}=+92 \mu \mathrm{m}$. These transitions represent points at which the lasing wavelength 
is extremely sensitive to cavity length. No hysteresis was observed beyond that attributed to the mechanical setup. It is noteworthy that the transition at $\Delta \mathrm{L}=+92 \mu \mathrm{m}$ corresponds to the threshold detuning at which the uniform pulse train develops a periodic modulation. Also of interest is the region of trichromatic emission between $L=L_{\text {opt }}+540 \mu \mathrm{m}$ and $\mathrm{L}=\mathrm{L}_{\text {opt }}+620 \mu \mathrm{m}$.

The structure of the emission spectra also changes significantly with cavity length detuning and Fig 33(a-d) presents a sampling of typical spectra in an abbreviated sequence for (a) $L$ $=\mathrm{L}_{\text {opt }}-35 \mu \mathrm{m} ;$ (b) $\mathrm{L}=\mathrm{L}_{\text {opt }} ;$ (c) $\mathrm{L}=\mathrm{L}_{\text {opt }}+92 \mu \mathrm{m}$ (the threshold for the periodic modulation) and (d) $\mathrm{L}=\mathrm{L}_{\text {opt }}+280 \mu \mathrm{m}$. Through the transitions noted above, the average power out of the dye laser does not change appreciably. In fact, as a rule, the average power is a fairly smooth function of detuning that peaks around $L=L_{\text {opt }}$ $+50 \mu \mathrm{m}$. Consequently, though the bandwidth and the peak heights of these spectra change, at times abruptly and drastically, the area under each of these curves is roughly constant.

As the cavity is further lengthened, the main peak of Fig. 33(d) diminishes and the shoulder of Fig 33(c) returns. Examples of the resulting spectra are shown in Fig. 34 for $L=L_{\text {opt }}+585 \mu \mathrm{m}$, which may be viewed as an example of trichromatic emission. The separation of the peaks is on the order of a few nanometers and therefore is in the range of peak separation reported by Hillman et al. [87] and Lawandy et al. [88] in their studies of bichromatic emission from $\mathrm{cw}$ dye lasers. 


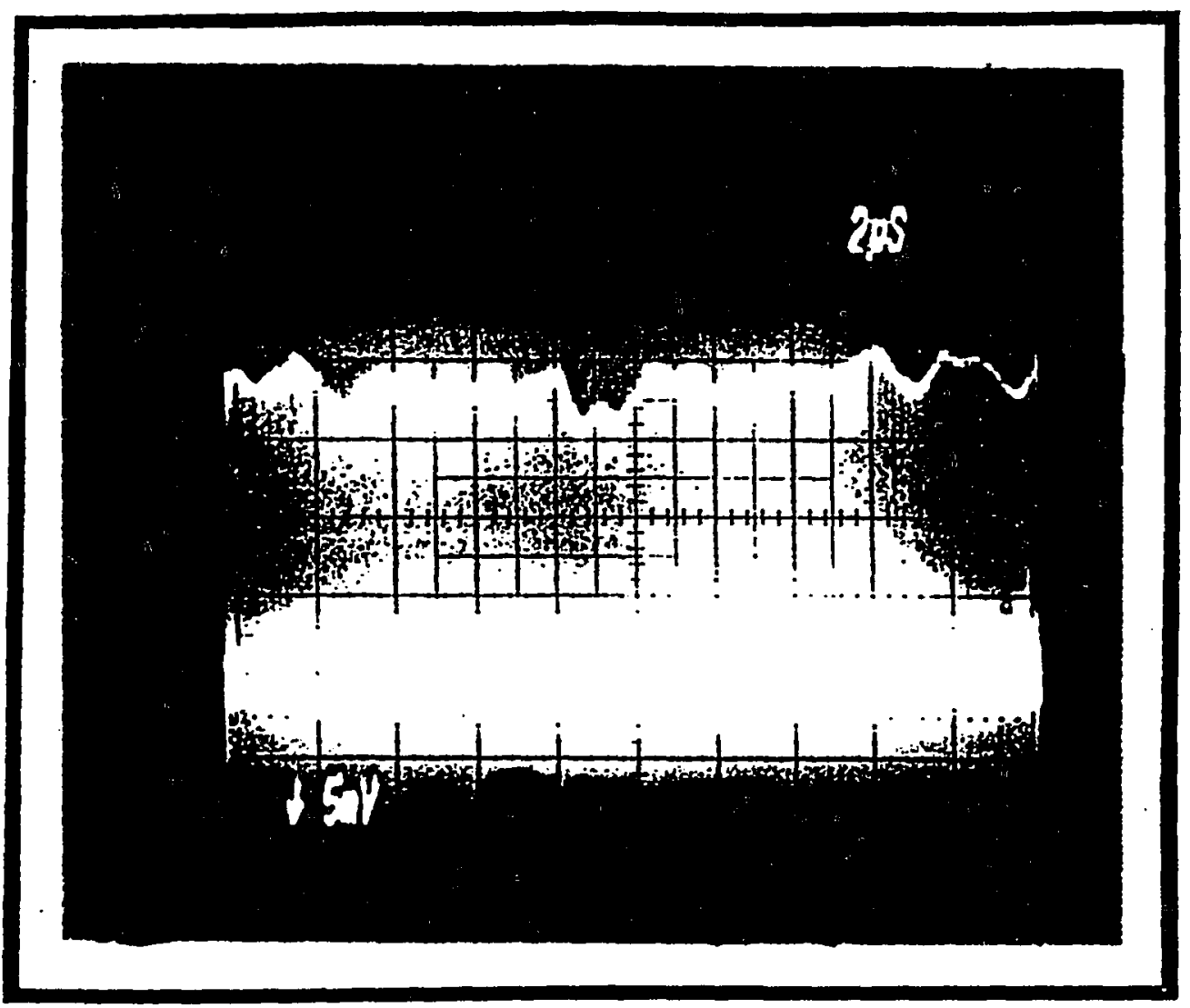

Figure 26a. SPML pulse train at optimum cavity length. 


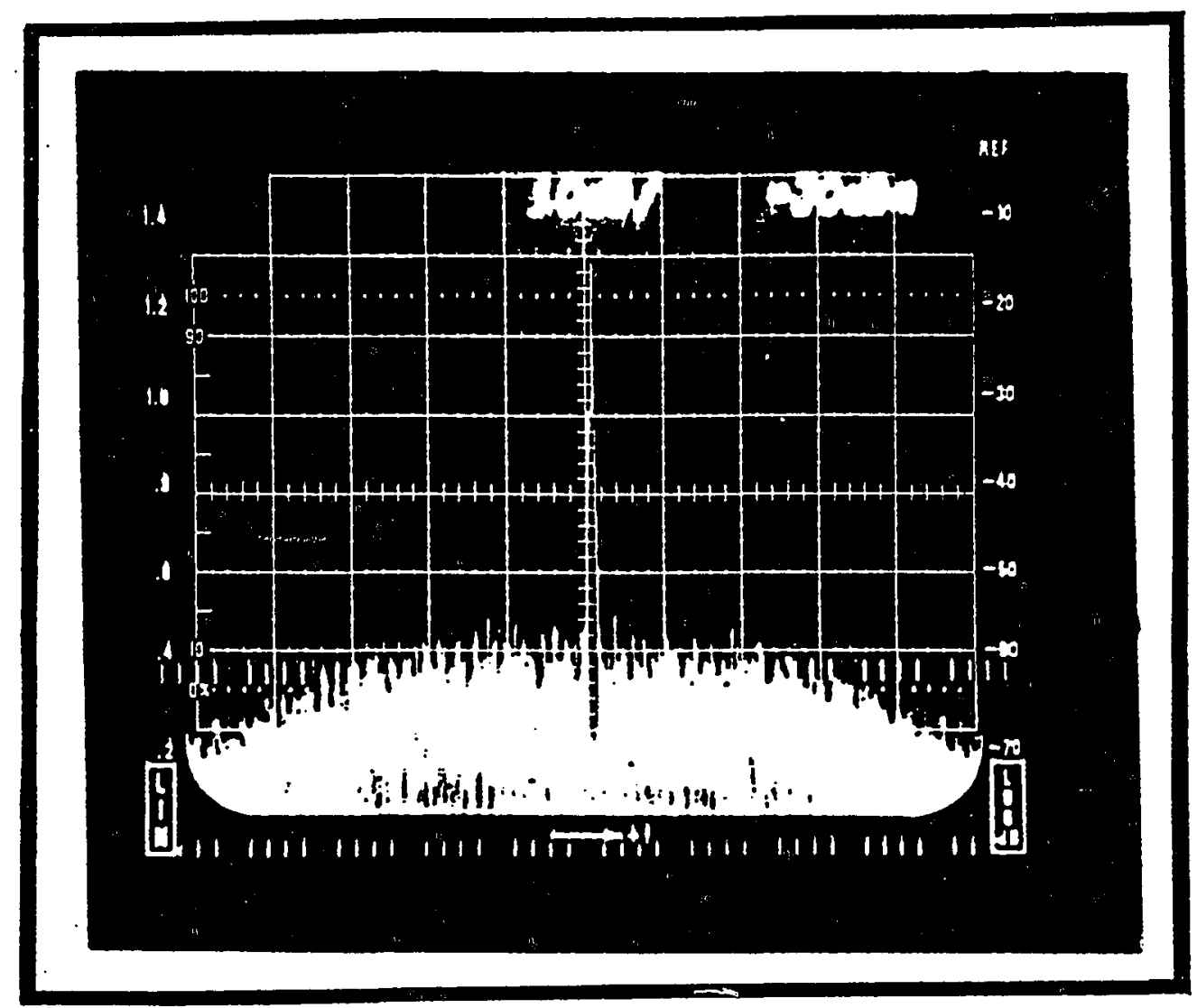

Figure 26b. SPML if spectrum at optimum cavity length. 


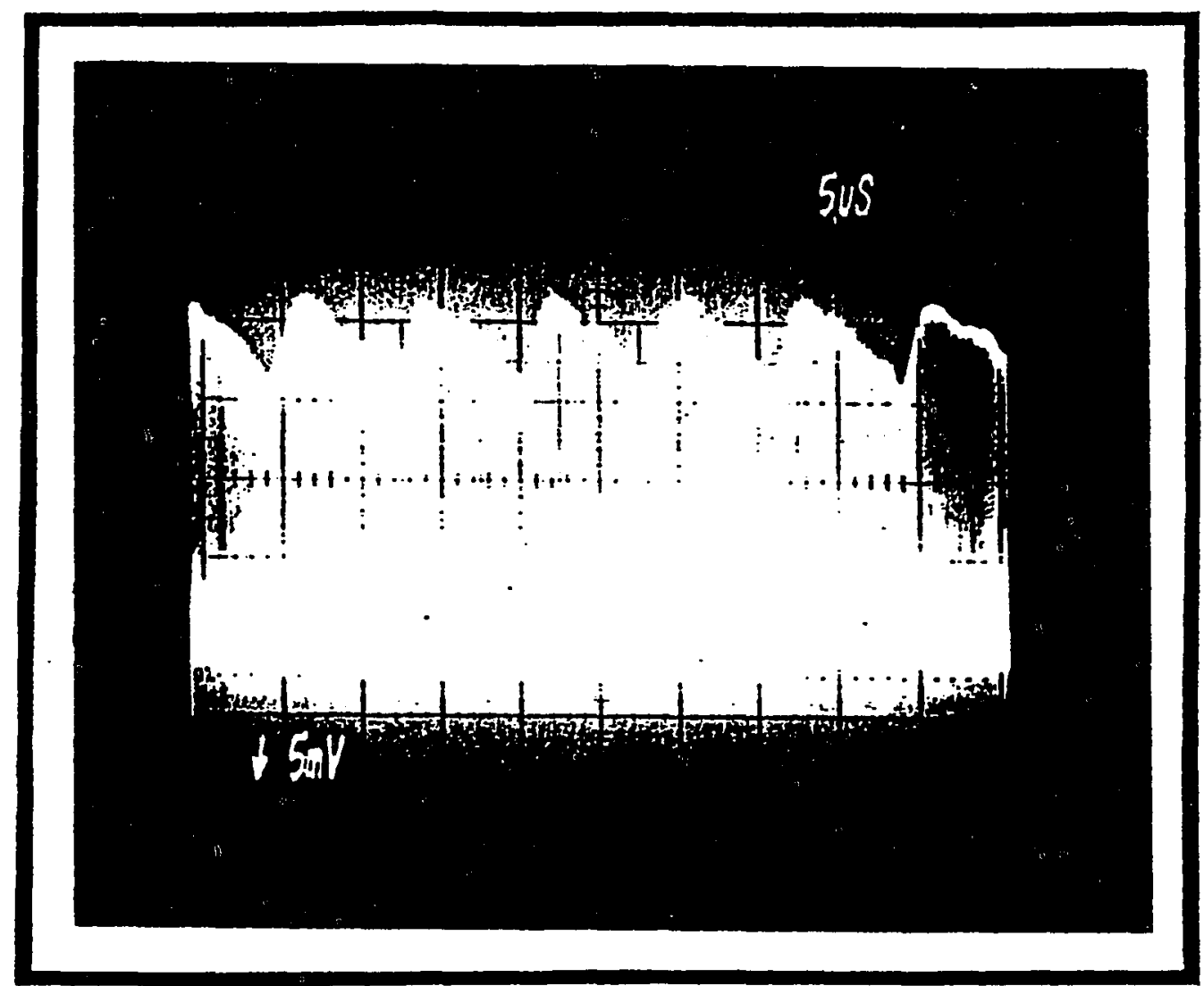

Figure 27a. SPML pulse train envelope at $\Delta L=125$ microns. 


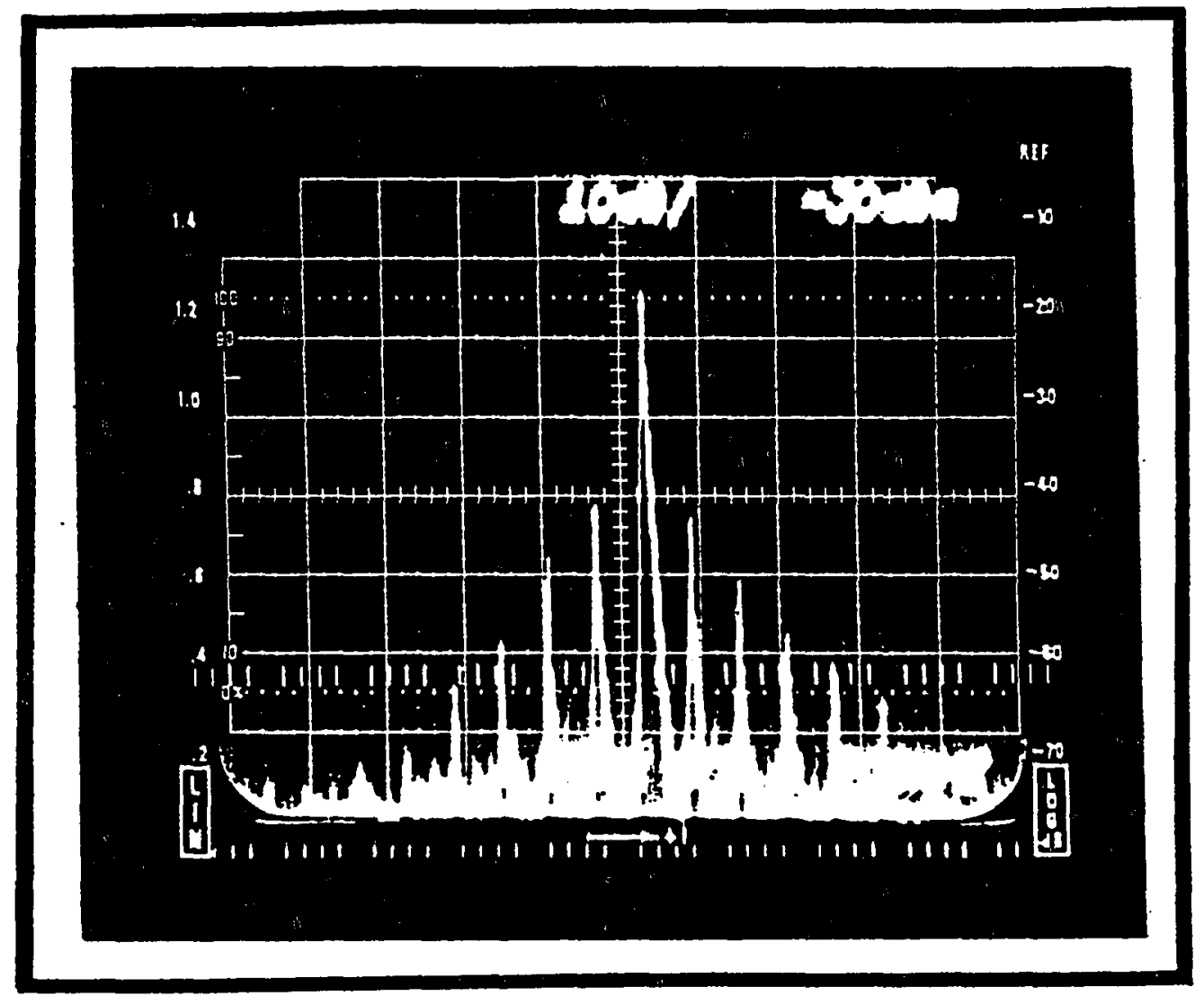

Figure 27b. SPML if spectrum at $\Delta L=125$ microns. 


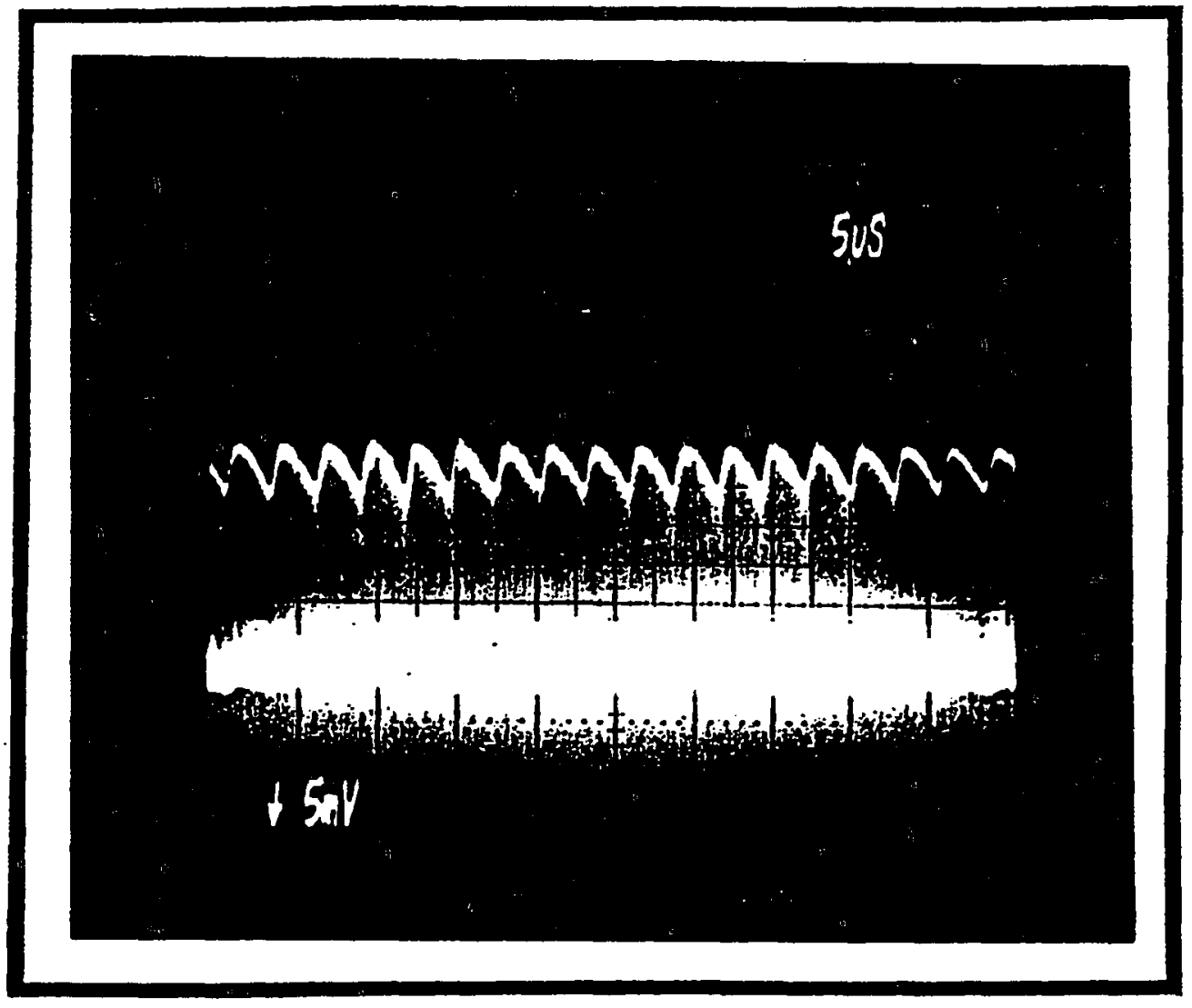

Figure 27c. SPML pulse train envelope at $\quad \Delta L=320$ microns. 


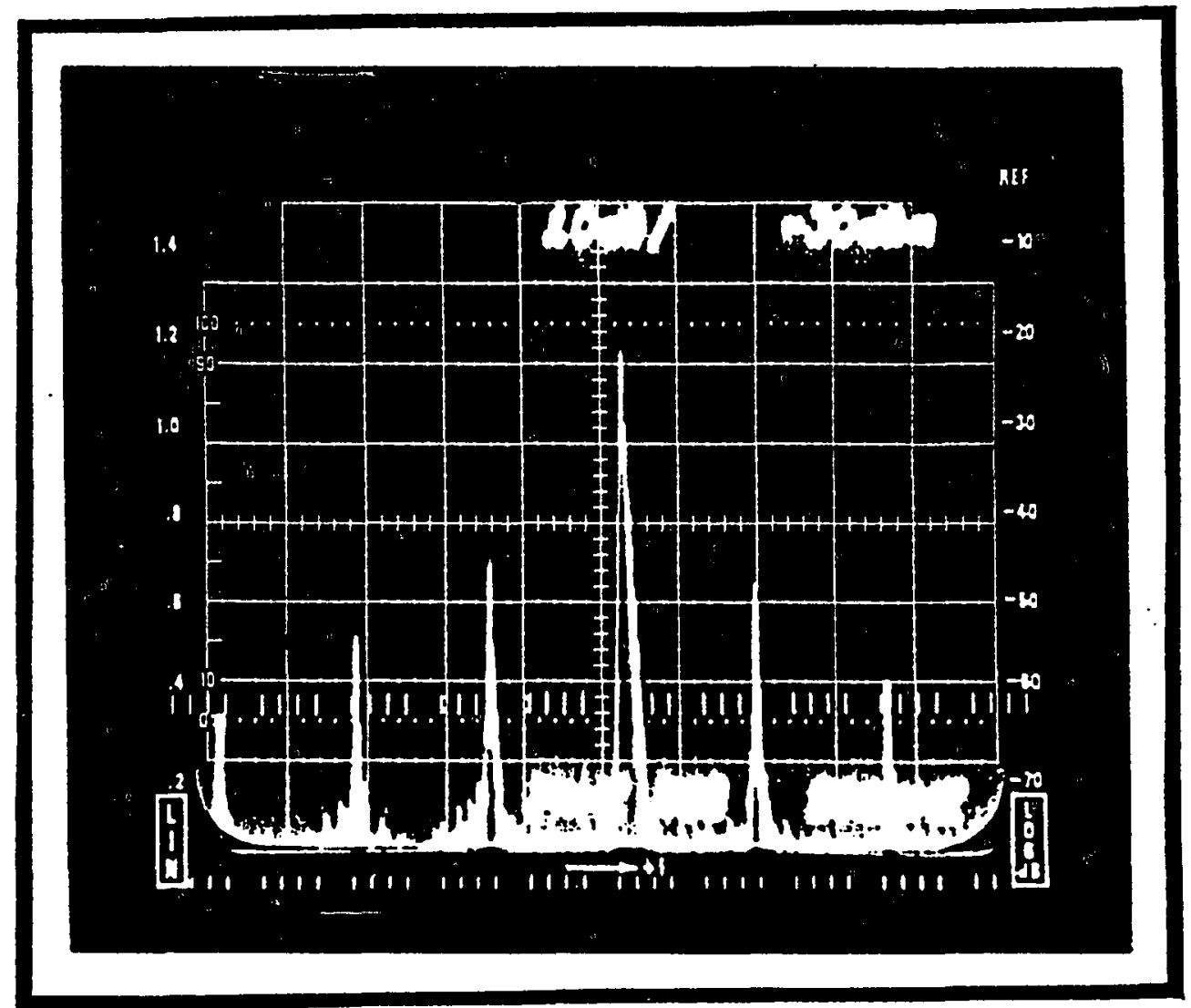

Figure 27d. SPML if spectrum at $\Delta \mathrm{L}=320$ microns. 


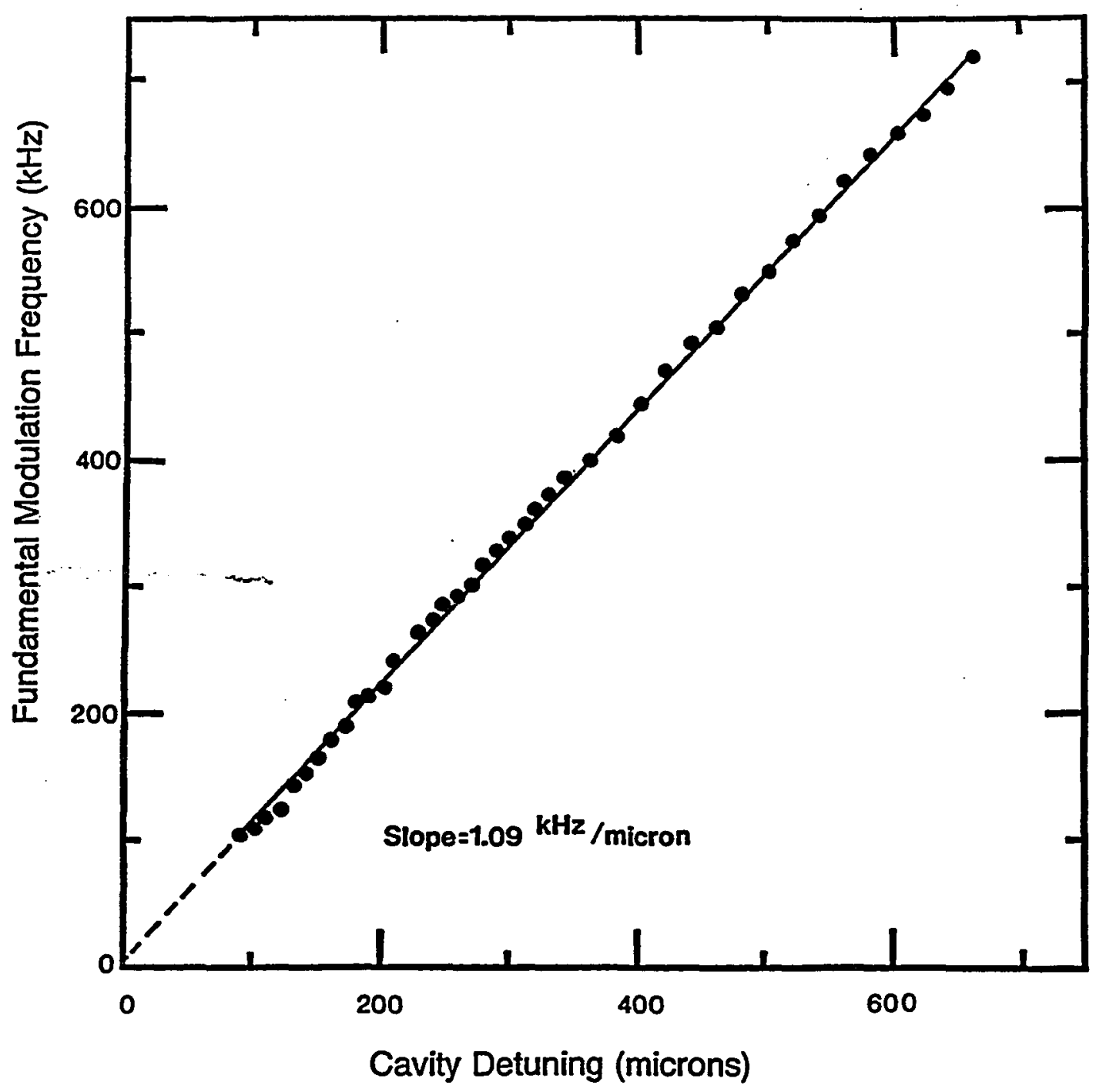

Figure 28. Modulation frequency versus detuning. 


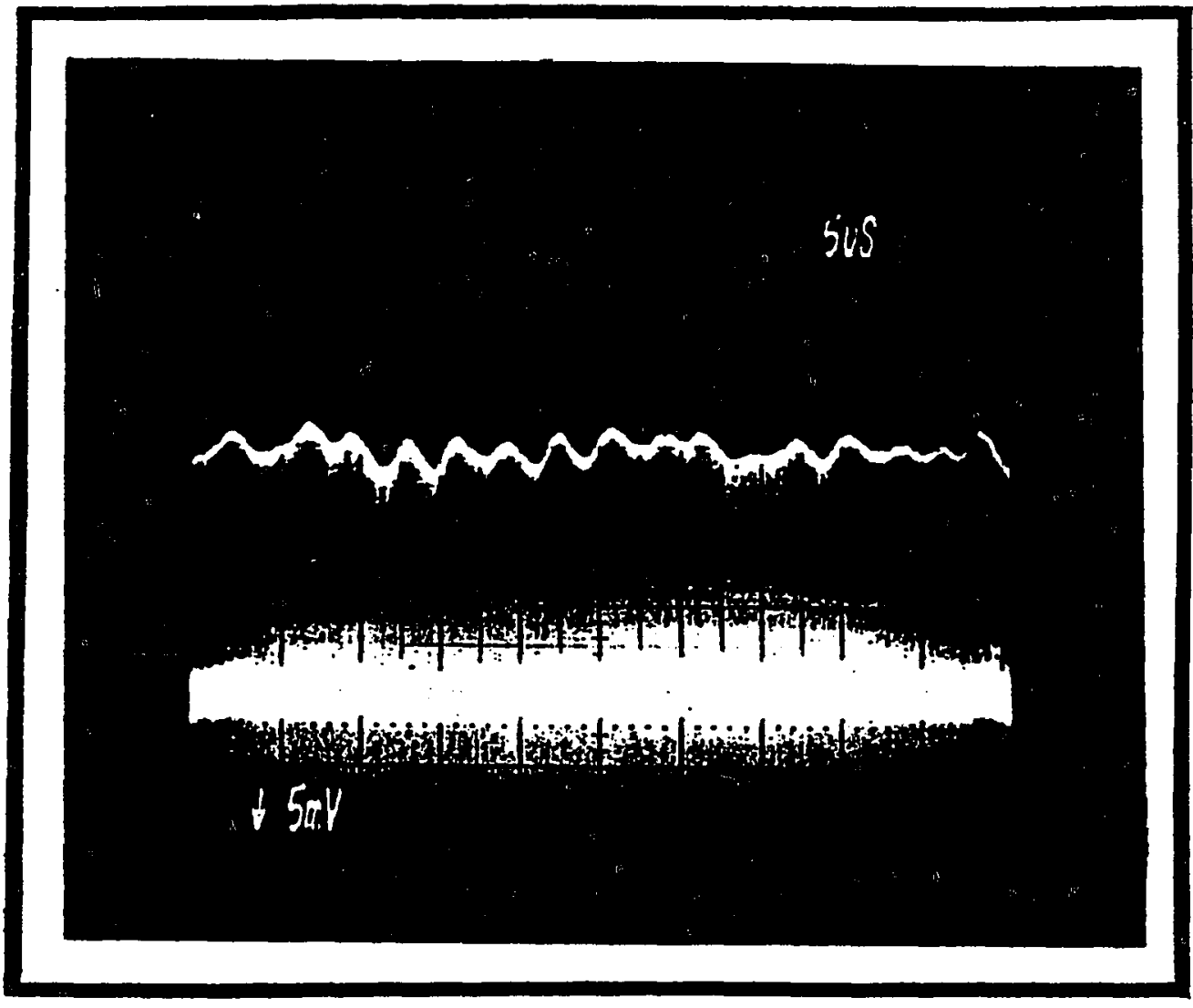

Figure 29a. SPML pulse train envelope at $\Delta L=-60$ microns. 


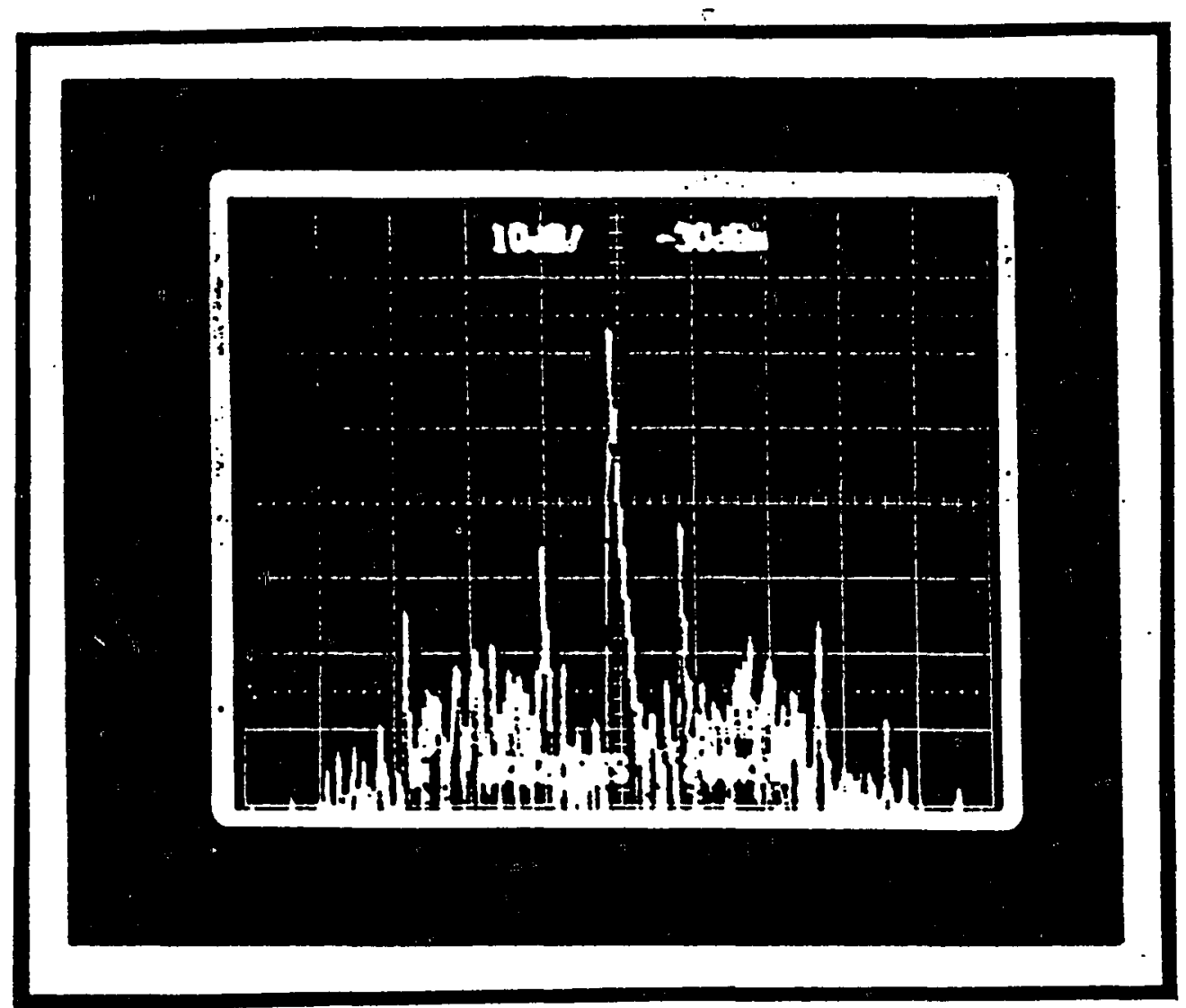

Figure 29b. SPML if spectrum at $\Delta L=-60$ microns. 


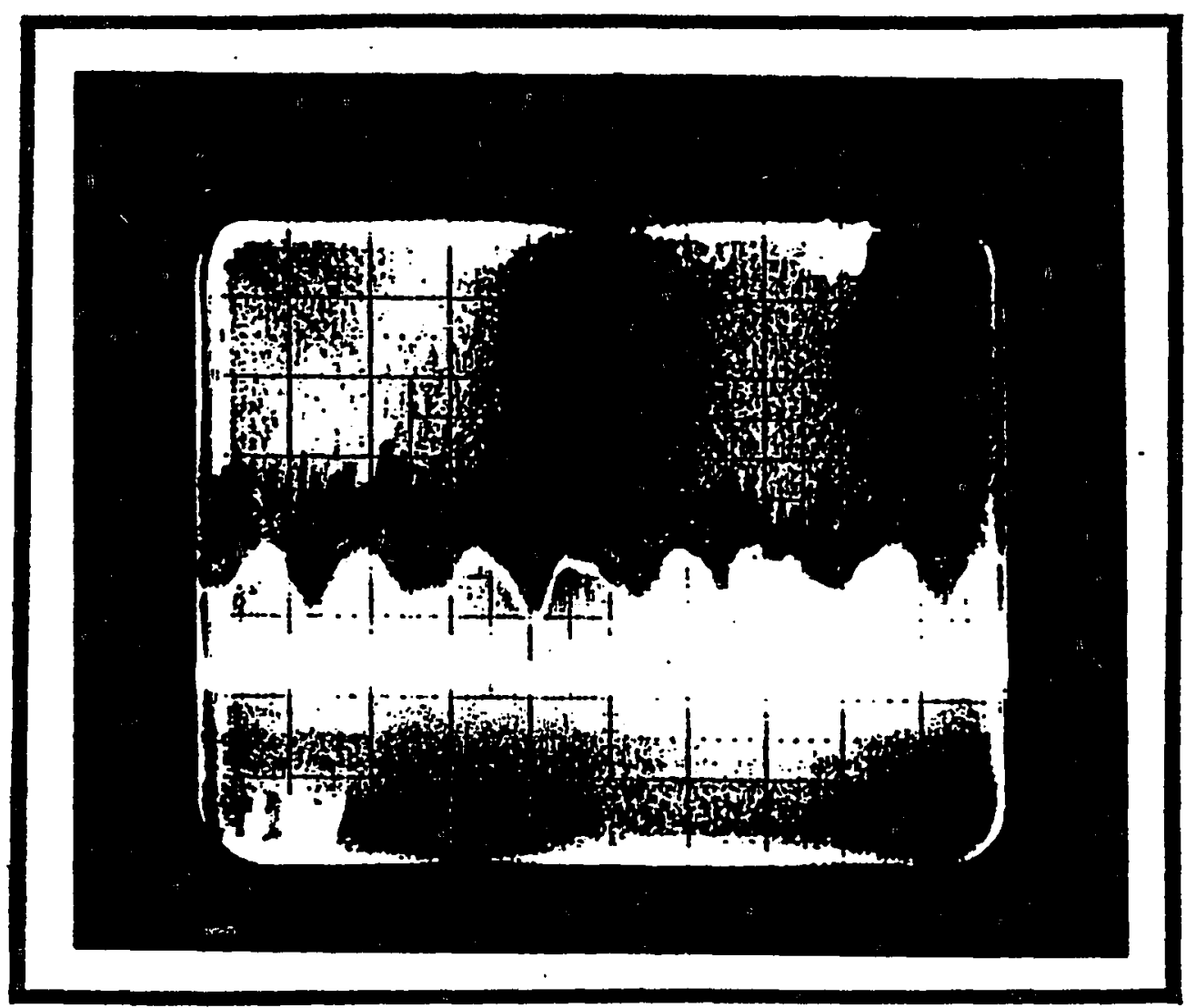

Figure 30a. SPML pulse train envelope at $\Delta L=-125$ microns. 


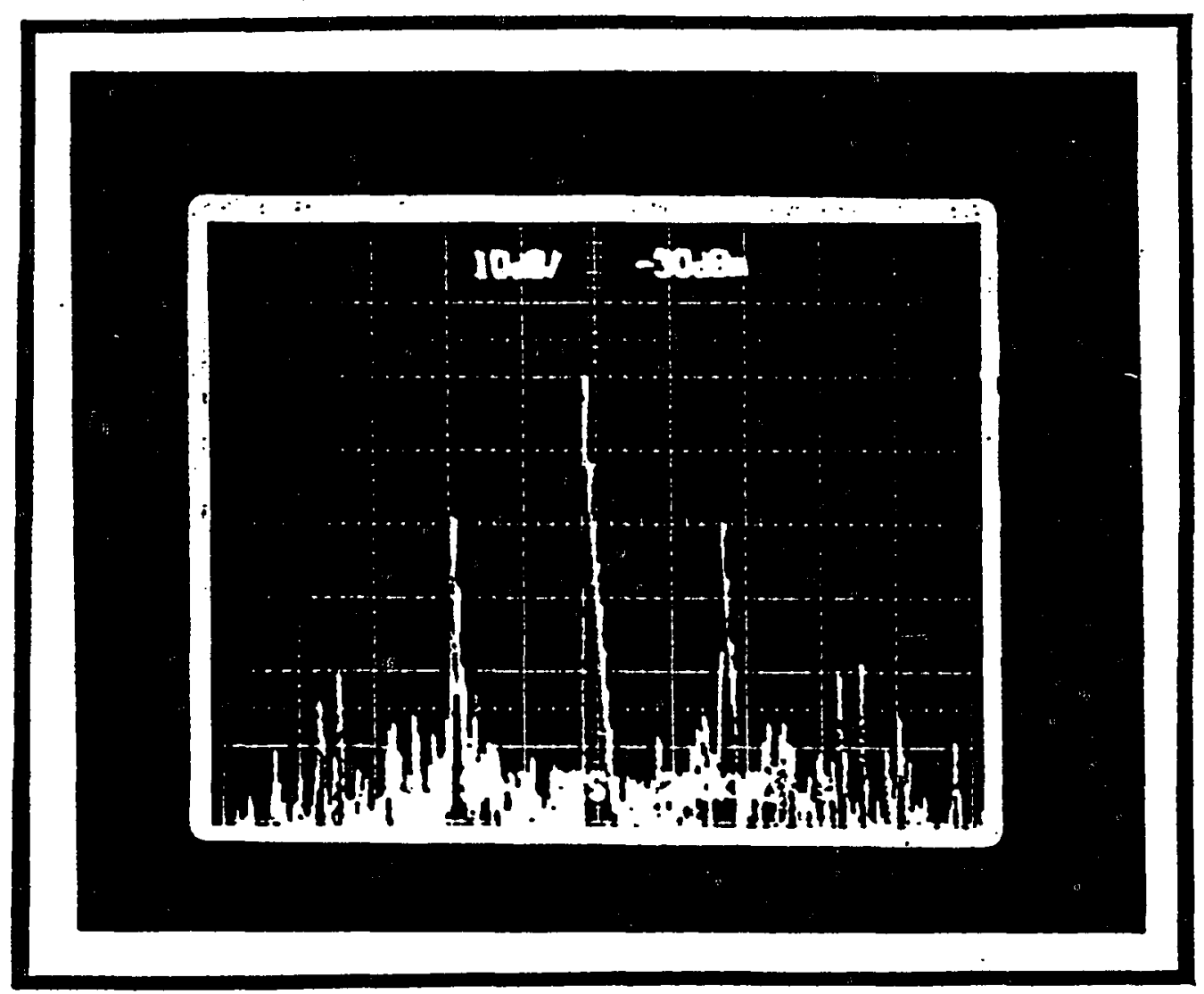

Figure 30b. SPML if spectrum at $\Delta L=-125$ microns. 


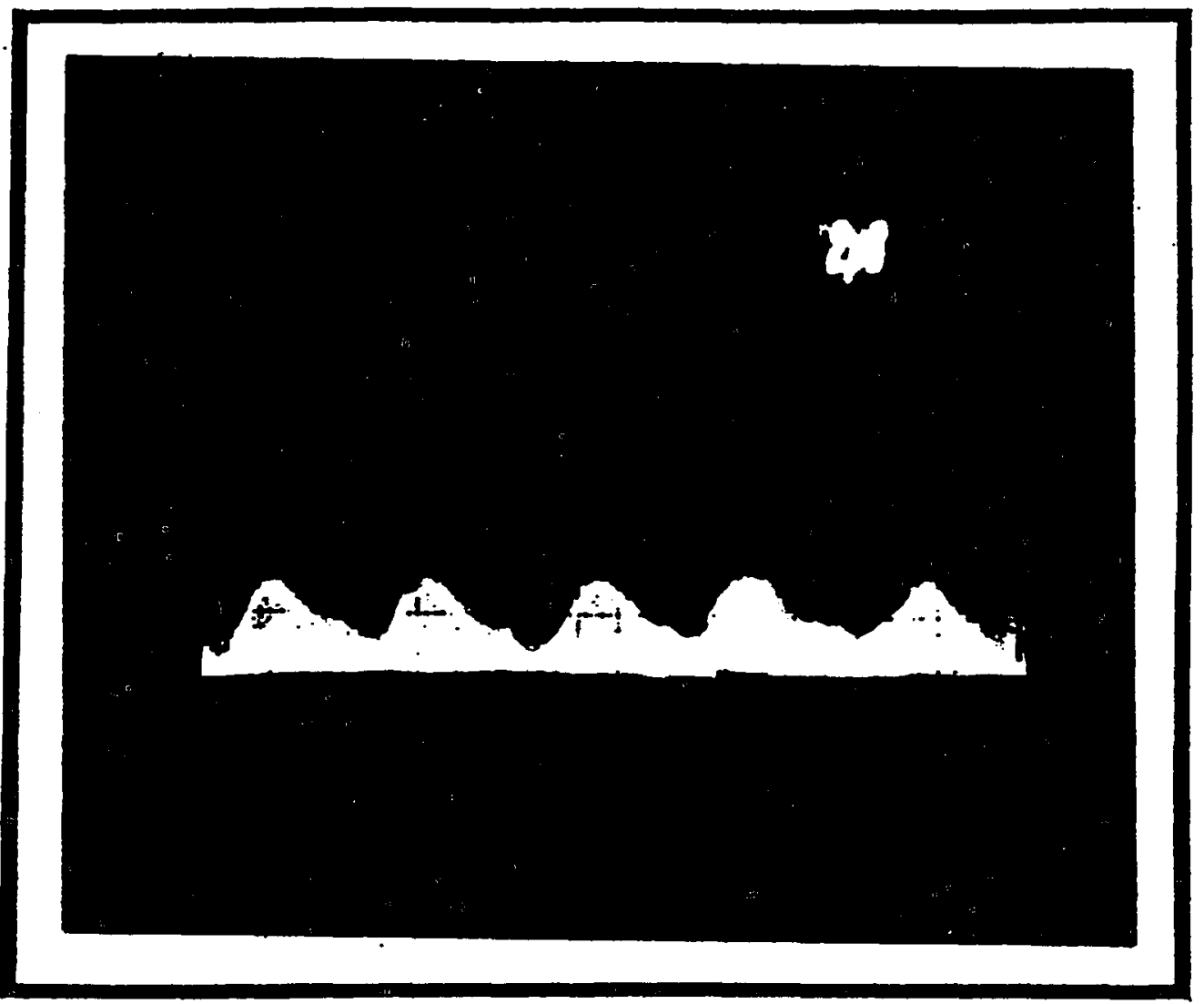

Figure 31a. SPML pulse train envelope at $\Delta L=-128$ microns. 


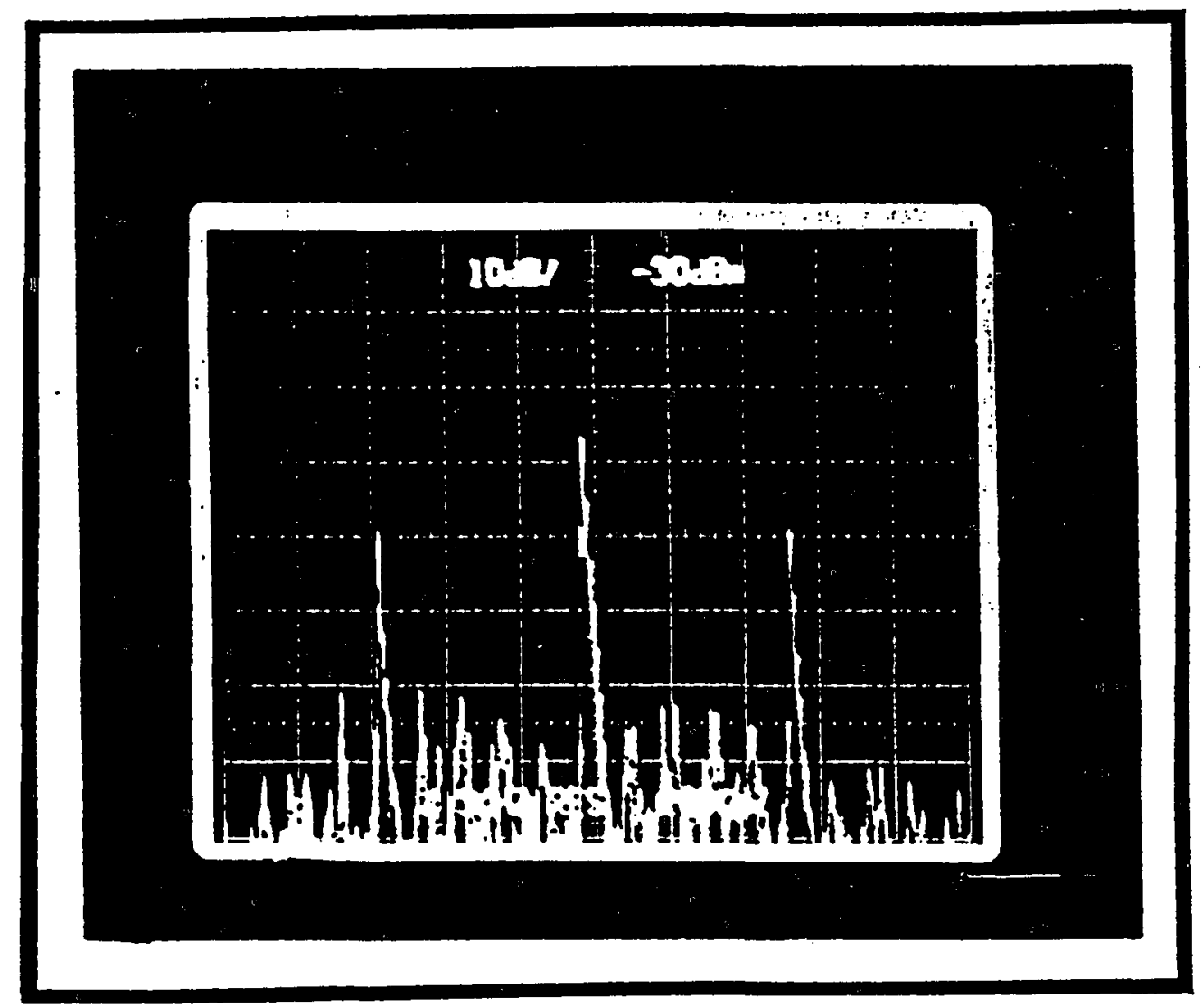

Figure 31b. SPML if spectrum at $\Delta L=-128$ microns. 


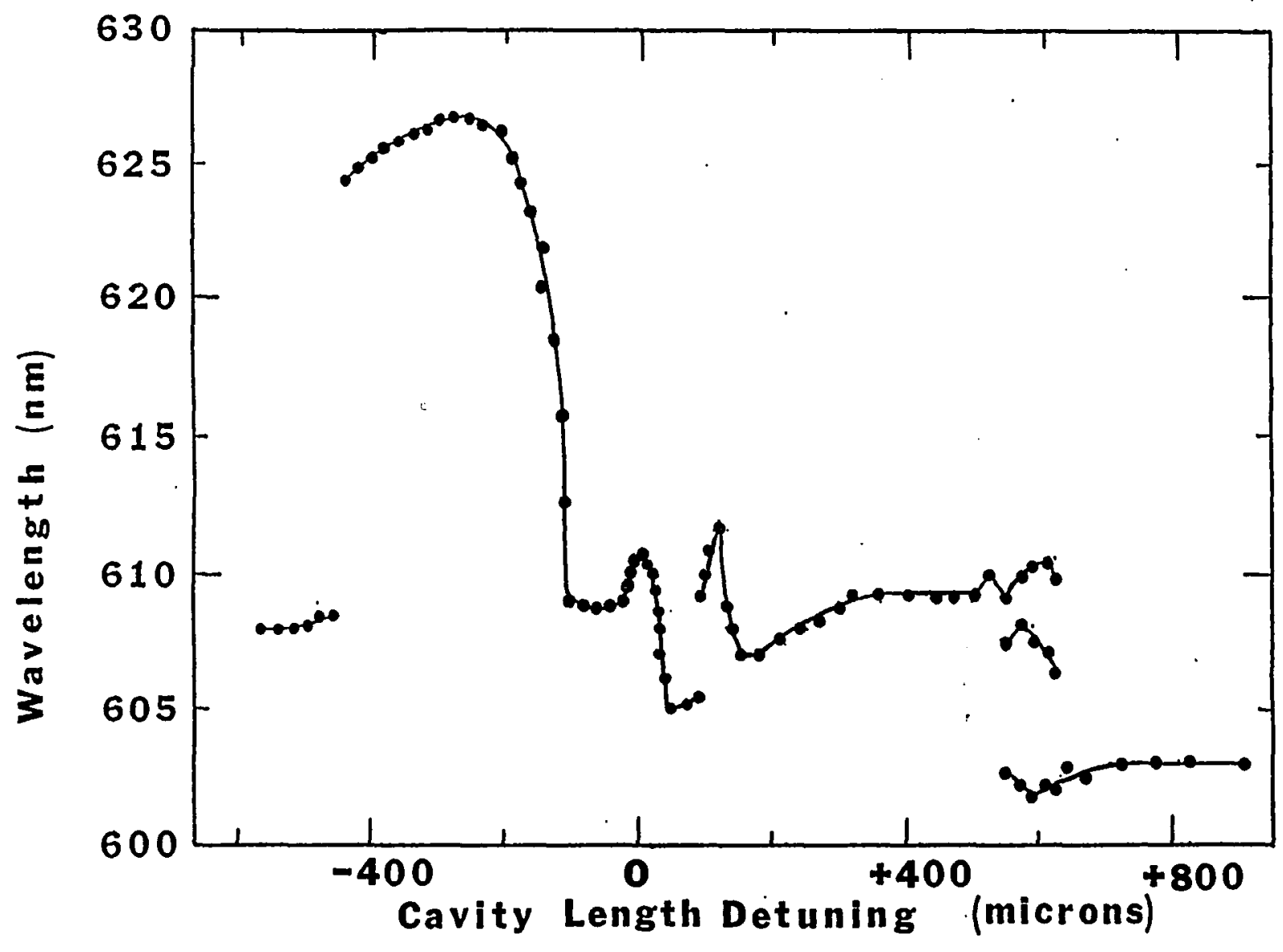

Figure 32. Peak lasing wavelength versus detuning. 


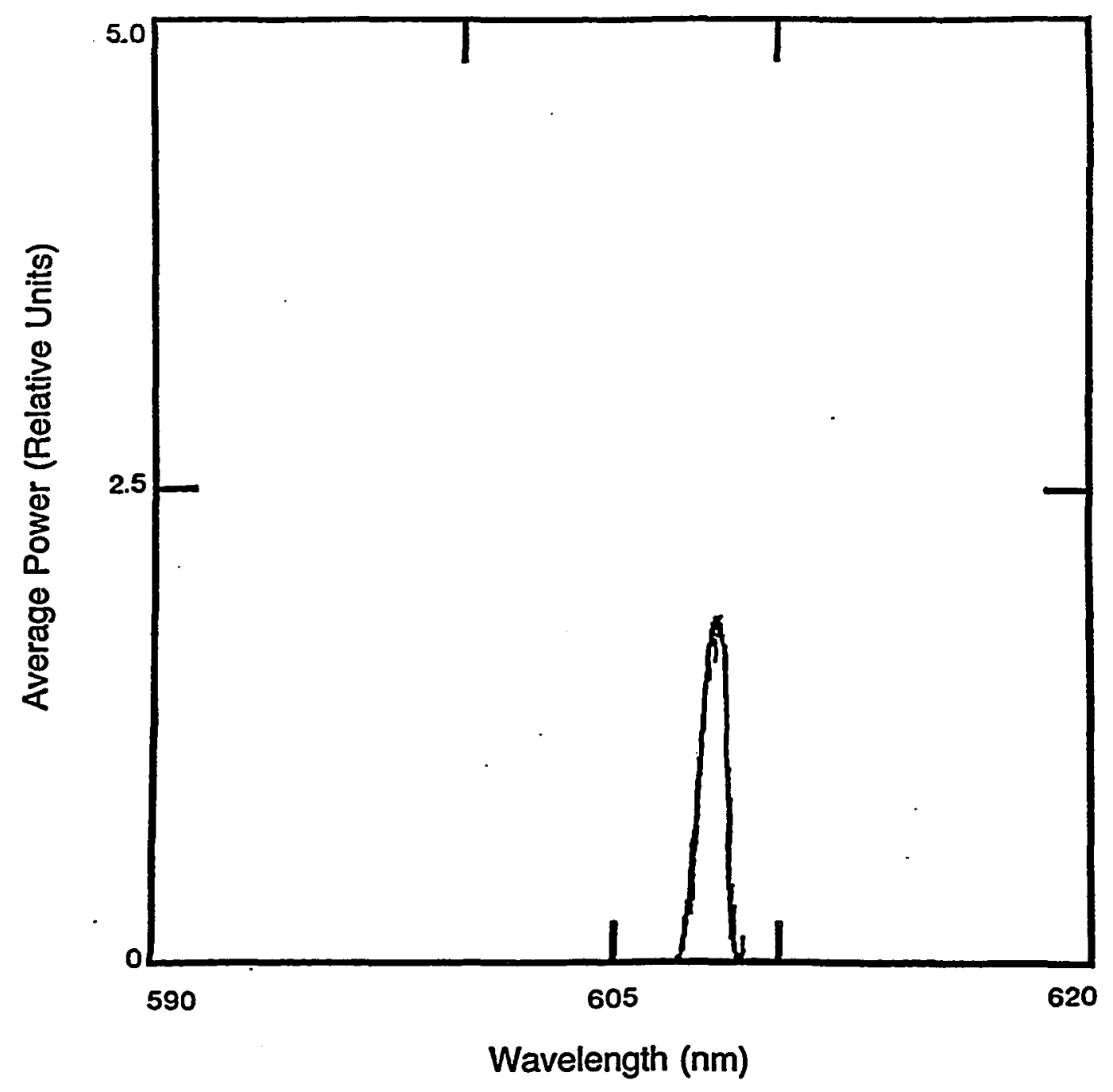

Figure 33a.Emission spectrum of SPML laser at $\quad \Delta L=-35$ microns. 


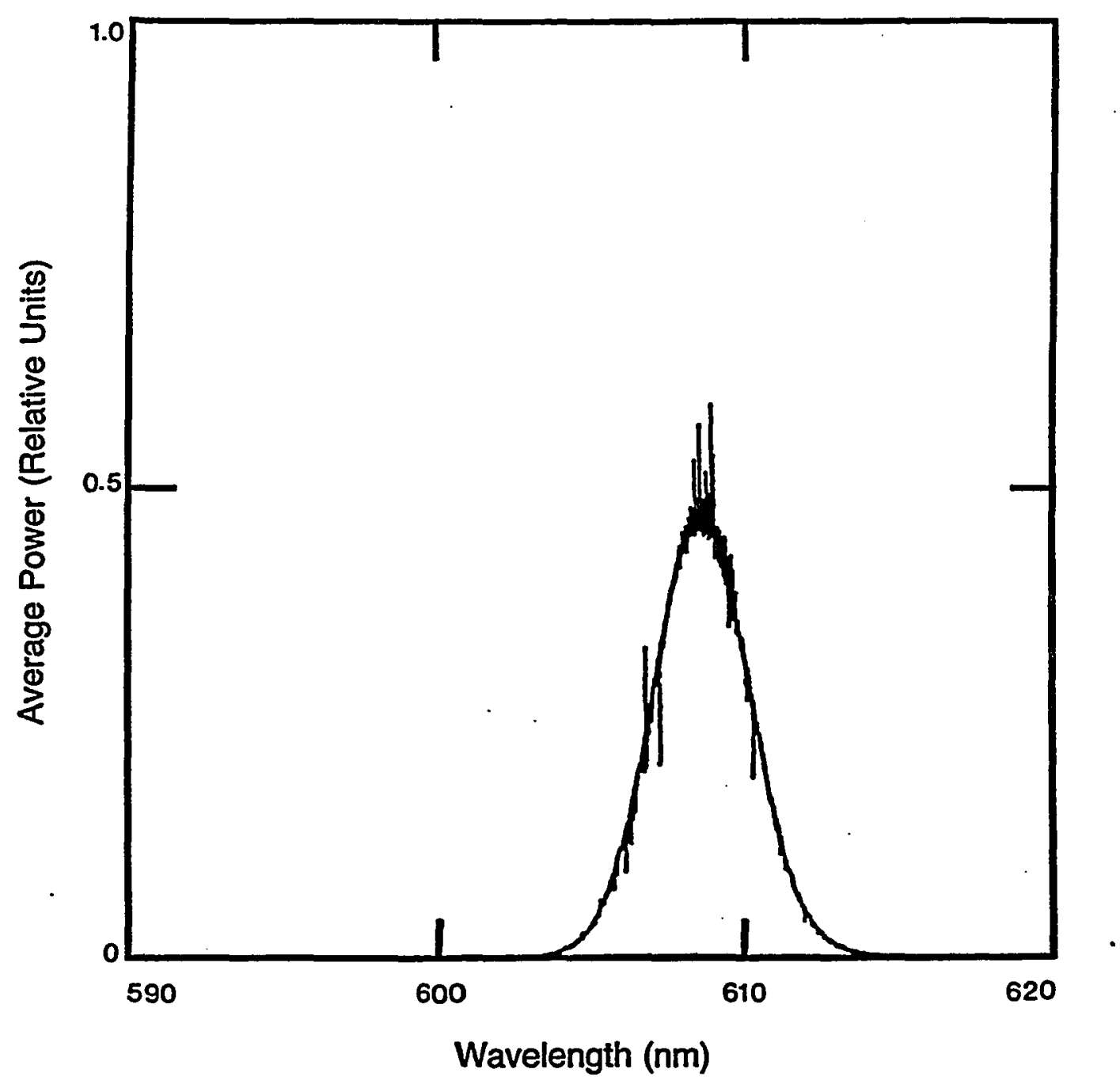

Figure 33b.Emission spectrum of SPML laser at optimum cavity length. 


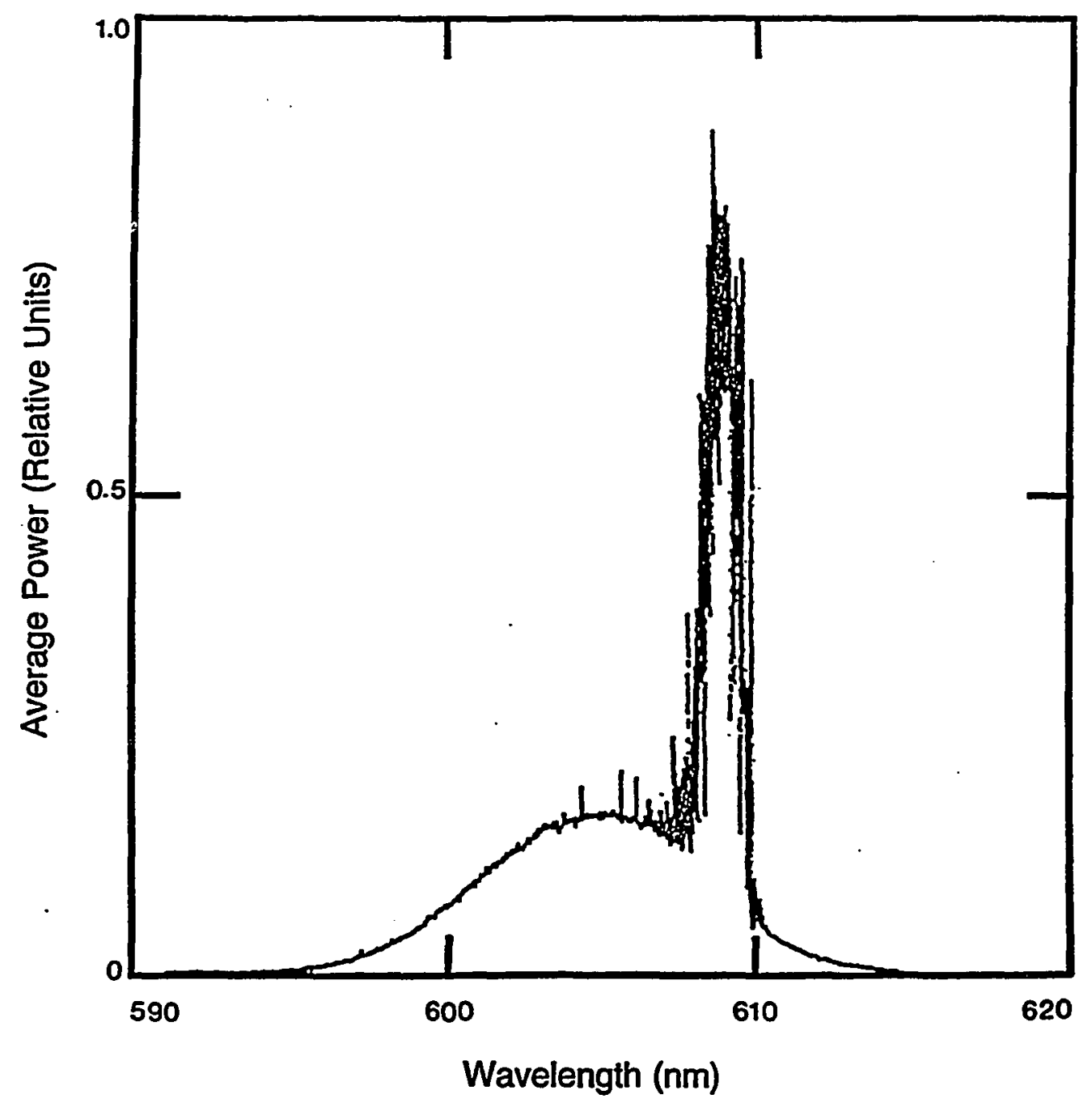

Figure 33c.Emission spectrum of SPML laser at $\Delta L=92$ microns. 


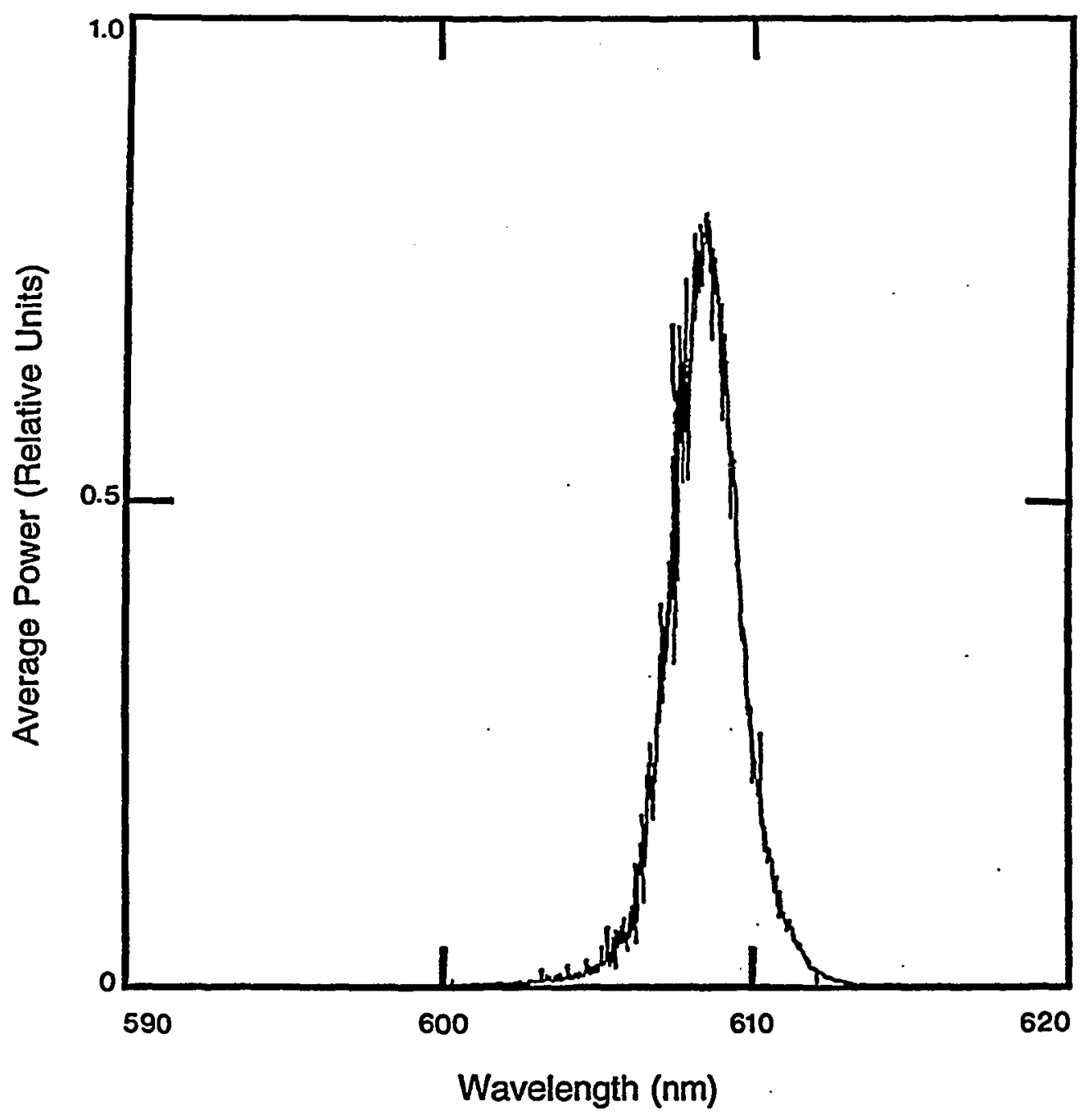

Figure 33d.Emission spectrum of SPML laser at $\quad \Delta L=280$ microns. 


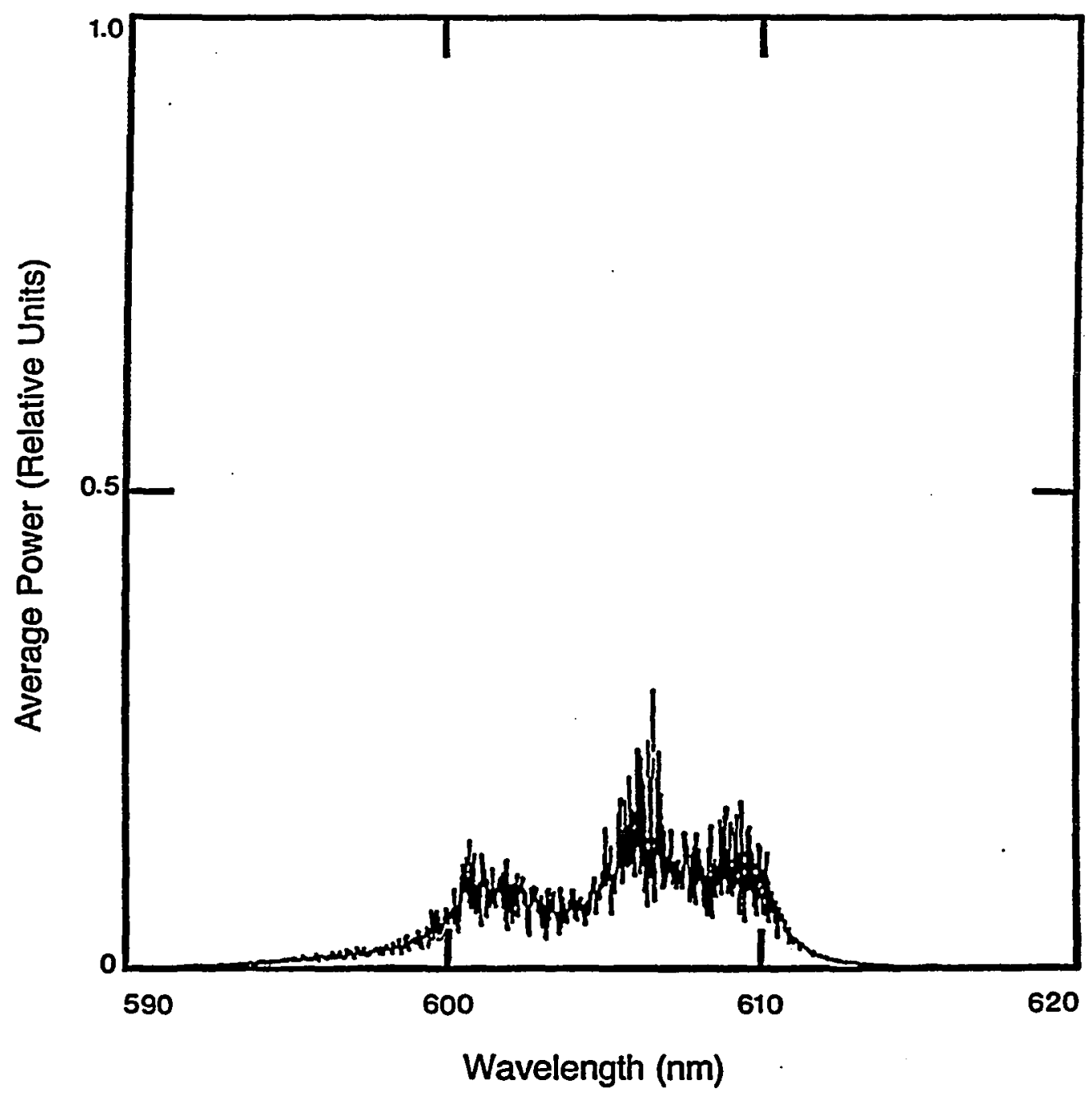

Figure 34.Emission spectrum of SPML laser at $\Delta L=\mathbf{5 8 5}$ microns. 
The effects reported here represent several different aspects of SPML operation. It would be fair to expect that the pulse train instabilities described here would arise from the nonlinear dynamics of short pulse formation where models that provide good agreement with pulse shape data have been derived without wavelength considerations [24]. Furthermore, New's work suggests that, because of spontaneous emission, the pulse train for an SPML laser is inherently unstable in certain length regimes [80]. More heuristically has been the work of Ogawa and Hamamura who have studied the chaos predicted by the Maxwell-Bloch equations under sinusoidal driving [89]. Of interest in a specific model of our SPML work would be the keystone role of the linear filter element in the formation of the erratic envelope, for without the filter, the pulse train is stable. That the relevant characteristic of the filter is its finite bandwidth (response time) is supported by the observation that the character and magnitude of the erratic envelope is independent of filter detuning, but increases for filters of decreasing bandwidths.

To contrast with the filter dependent erratic envelope in the region about $\mathrm{L}_{\mathrm{opt}}$ is the periodic modulation depicted in Fig. 27 that occurs for long cavities whether the filter is present or not. Associated with this effect is the linear shift of the modulation frequency with cavity length increase shown in Fig. 28. Importantly, the threshold detuning value of $+92 \mu \mathrm{m}$ and the best fit slope of $1.09 \mathrm{kHz} / \mathrm{\mu m}$ are independent of the filter. Associated with this transition, there is the abrupt change in wavelength (see Fig. 32) and the double-humped emission of Fig. 33(c) that characterize the critical detuning of $+92 \mu \mathrm{m}$. This marked 
transition in the emission spectra of the dye laser at the onset of the periodic amplitude modulation of the pulse train suggests that wavelength and band considerations are also important in the dynamical model. To clarify these issues, there is more work to be done on the effects of bandwidth limiting tuning filters on the output of SPML lasers.

In the absence of a rigorous theoretical model, one may speculate on plausible explanations for the shifts in wavelength with cavity detuning. One possibility would be that the shifts arise from the dispersion of the rhodamine dye jet. Given that the gain from the argon laser arrives periodically with a fixed frequency $v_{a r}$, any stable pulse propagating in the cavity has to satisfy the condition

$$
k v_{a r}=c / 2 n(\lambda) L
$$

where $c$ is the speed of light, $n$ is the effective wavelength dependent index of the cavity, $L$ is the cavity length, and $k$ is an integer. The propagating pulse can then fulfill equation (150) if it changes its wavelength to keep its effective cavity length constant. Since most of the cavity is tree space, $n(\lambda)=n_{0}\{1-1 / L\}+(1 / L)$ $n_{d}(\lambda)$, and (150) may be rewritten as,

$(l / L) n_{d}(\lambda)=c / 2 L v_{a r}-n_{0}\{1-l / L\}$

Here, $n_{0}$ is the index of free space, $n_{d}(\lambda)$ is the dispersive index of the dye, $l$ is the length of the jet, and in accordance with our setup, $\mathrm{k}=1$. However, since the dispersion of the dye is scaled by 
$1 / 1 \approx 10^{-6}$, this explanation can not account for the $25 \mathrm{~nm}$

variation in $\lambda$. In other words, since the dye jet is only about 200 um long, its index must about double to compensate for a similar shift in cavity length.

More fundamentally, it is the group velocity,

$v_{g}=c /(n+v d n / d v)$

and not the phase velocity, $c / n$, that one needs to consider in (150) above. In (152), $v \mathrm{dn} / \mathrm{dv}$ is the dispersion which, for a homogeneous laser operating near line center is

$v_{v} \mathrm{dn} / \mathrm{d}_{v}=c g(v) /\left(2 \pi \Delta v_{h}\right)$

Here, $g(v)$ is the small signal gain and $\Delta v_{h}$ is the homogeneous linewidth. For our laser $v d n / d v$ may be appreciable - on the order of $0.4-0.8$. Consequently, a variation in group velocity might contribute to the wavelength variations observed here.

Alternatively, a simple band model argument can also lead to wavelength shifts. The upper lasing state, $S_{1}$, of the dye, is actually a $30 \mathrm{~nm}$ band of vibrational sublevels with an intraband relaxation time on the order of a few psec [24]. Since the $s_{1}-S_{0}$ transition time is about $5 \mathrm{nsec}$, the excited dye molecules typically accumulate at the bottom of the band and it is a good approximation, under normal lasing conditions, to ignore the band structure altogether. Nonetheless, in a synchronously pumped configuration, a shortening of the cavity could cause the pulse to arrive before the excited molecules have cascaded to the bottom of 
the $S_{1}$ band. This blue shifts the lasing wavelength. Given a $30 \mathrm{~nm}$ bandwidth and an intraband relaxation time of $4 \mathrm{psec}$, this picture predicts roughly a $5 \mathrm{~nm}$ shift in wavelength for a $100 \mu \mathrm{m}$ cavity detuning. This figure is close to the slope at several places of the wavelength detuning curve of Fig. 32. Of note here, is the recent study by Hong and Haken [90] that suggests that the band structure of dye molecules may account for the spectral behavior observed in [87] and [88].

More experimental work needs to be done on the multipeaked emission spectra. It is tempting to associate our data with the bichromatic emission observed by Hillman et al. [87] and Lawandy et al. [88], but the dramatically different experimental configurations and our relatively broad, non-distinct spectral peaks are significant obstacles to such an association. The 40 psec pulse $(1.2 \mathrm{~cm})$ and our cavity geometry makes our laser essentially unidirectional in the pulse/active medium interaction. Thus, no puise shortening, spectral splitting dispersion grating can be set up.

While the effects reported here are pertinent characteristics of a synchronously pumped mode-locked system, it is not anticipated that these effects will be easy to reproduce with a single simple theoretical model. Existing models for SPML lasers are already highly complex, and in most cases they omit the nonperiodic behavior, bandwidth limiting filters and dye spectral characteristics that are apparently fundamental to these experimental observations. 


\section{CHAPTER VI}

\section{CONCLUSIONS}

The ambit of this dissertation was the furthering of the technology of synchronously pumped mode-locked dye lasers, an important source of ultrashort optical pulses. In particular, we have studied ways to produce pulses that are shorter, more intense and more consistent on a pulse-to-pulse basis. Throughout this work we have attempted to balance theory with experiment, consistently checking the former with the latter. While this is the proper scientific method associated with a comprehensive study, this dissertation does not pretend to be comprehensive. Much work remains to be done before a definitive discussion of SPMI dye lasers becomes appropriate. This dissertation is concise in that it is composed only of a set of original studies that may be read either in isolation or in sum. There is no one section containing background material and summarizing the many contributions of others. There is, however, a detailed reference list.

The first study entitled, "Pump pulse effects in synchronously pumped mode-locked dye lasers," shows, for the first time, how to optimize a SPML laser with respect to the parameters associated with the pump pulse. The model presented is in good agreement with our own experiments and with others reported in the general literature. This study provides a clear theoretical exposition of the most advanced SPML dye lasers and demonstrates ways to improve the peak intensity and the 
pulsewidth of the output pulse. A paper similar to this chapter has been published in the March 1989 issue of the Journal of the Optical Society of America B.

The second study, "Theory of a synchronously pumped mode-locked dye laser in the short pump pulse limit" uses the results of chapter two to develop an intuitive, analytic theory of SPML operation. In this work simplified and qualitative mathematical expressions are derived that allow immediate laboratory progress in areas such as pulse shortening and stability. These mathematical formulae suggest that gain swithing, rather than gain modulation, is the dominant physical process in SPML pulse production. This work has appeared in the June 1989 issue of the Journal of the Optical Society of America B.

The third study, "Coherence effects in hybridly mode-locked lasers" is the first semiclassical theory explaining synchronously pumped mode-locked dye lasers with intracavity saturable absorbers. This theory offers quantitative agreement with experiments and suggests further techniques to improve peak intensity and pulsewidth. The pump pulse optimization of hybridly mode-locked lasers may be found in the March 15, 1989 issue of Optics Letters under the title, "Pump pulse effects in hybridly mode-locked lasers." The remainder of chapter four has been submitted to the IEEE Journal of Quantum Electronics.

In chapter five and the appendix are experimental examinations of the pulse train instability associated with SPML dye lasers and loss modulated mode-locked argon ion lasers. The issue of stability in these lasers is an important one that has not properly been addressed; many applications demand pulse-to-pulse stability in addition to short pulses, however most research groups have, reasonably enoush, focussed on the primary issue of 
generating short pulses. Noise may arise from a variety of sources. The usual mechanical sources include temperature shifts and vibrations. Pump source jitter is an important factor since the SPML process will amplify this irregularity considerably. In addition to these external factors, our experimental work points out a fundamental instability associated with SPML systems and with actively mode-locked lasers in general. In current SPML systems this instability is the largest source of noise and remains uncorrected. The author feels confident, albeit from nonrigorous intuition, that the forced, nonlinear dynamical formalism used in this dissertation will eventually explain this pulse train instability. This pulse train instability work may be found in the Journal of the Optical Society of America B. under the titles, "Spectral behavior and pulse train instabilities of a synchronously pumped mode-locked dye laser," (May, 1988) and "Pulse train instabilities in a mode-locked argon ion laser: experimental studies," (November, 1987).

This dissertation has also tried to further our understanding of the physics of synchronously pumped mode-locked dye lasers. Laser physics has been a rapidly developing, extremely successful field of study. Few other quantum mechanical devices benefit from as firm an understanding of the basic tenets - certainly neither semiconductors nor superconductors. The recent mysteries - the unsolved problems - of laser physics have included laser instabilities and mode-locked lasers. Each of these effects have provided an extreme test of the principles. Through dilligent and inspired effort, many of the questions associated with laser instabilities have been answered. Lasers are now being used to study the deeper, more general, issues of deterministic chaos or turbulence. That the same paradigm also serves to explain the 
extreme issue of laser "mode-locking" is a recent and profound result. The success of these models lies not only in their ability to quantitatively predict the output characteristics of these important devices. Nor does it lie solely in their ability to pinpoint technological improvements. The great success of the theories discussed in this dissertation is that they have furthered the basic laser paradigm and employed it to explain some of the most exotic laser devices. Many early explanations of "mode-locked" lasers had little relation to traditional laser theory. The scientific value of a paradigm, however, lies in its defeat; the point where a theory fails is the point where new physical knowledge begins. Happily and unfortunately, then, it appears that mode-locked lasers are on the verge of being well understood. Optimization of this type of laser with respect to a given application may follow. 


\section{REFERENCES}

[1] W. H. Glenn, M. J. Brienza and A. J. DeMaria, "Mode locking of an organic dye laser," Appl. Phys. Lett., 12, 54-56 (1968).

[2] D. J. Bradley and A. J. F. Durrant, "Generation of ultrashort dye laser pulses by mode locking," Phys. Lett. A, 27, 73-74 (1968).

[3] B. H. Soffer and J. W. Lin, "Continuously tunable picosecondpulse organic-dye laser," J. Appl. Phys., 39, 5859-5860 (1968).

[4] C. K. Chan and S. O. Sari, "Tunable dye laser pulse converter for production of picosecond pulses," Appl. Phys. Lett., 25, 403-406 (1974).

[5] J. M. Harris, R. W. Chrisman and F. E. Lytle, "Pulse generation in a cw dye laser by mode-locked synchronous pumping," Appl. Phys. Lett., 26, 16-18 (1975).

[6] A. M. Johnson, R. H. Stolen and W. M. Simpson, "80x singlestage compression of frequency doubled Nd:YAG laser pulses," Appl. Phys Lett., 44, 729 - 731 (1984).

[7] A. M. Johnson and W. M. Simpson, "Tunable femtosecond dye laser synchronously pumped by the compressed second harmonic of Nd:YAG," J. Opt. Soc. Am. B, 2, 619-625 (1985).

[8] (a) J. D. Kafka and T. Baer, "A synchronously pumped dye laser using ultrashort pump pulses," Proc. SPIE, vol 533 Ultrashort Pulse Spectroscopy and Applications ed by M. J. Soileau, 38-45 (1985). (b) Technical product information for Spectra Physics model 3800 mode-locked Nd:YAG, model 3695 pulse compressor and model 3500 SPML dye laser (1988).

[9] D.J. Kuizenga and A.E. Siegman, "FM \& AM modelocking of the homogeneous laser - part 1: theory," IEEE J. Quant Electron., $\mathrm{OE}-6,694-708$ (1970).

[10] H. Haus, "A theory of forced mode-locking," IEEE J. Quant. Electron, QE-11, 323-330 (1975). 
[11] N. J. Frigo, T. Daly and H. Mahr, "Study of a forced mode-locked cw dye laser," IEEE J. Quant. Electron., QE-13, 101-109 (1977).

[12] C. P. Ausschnitt, R. K. Jain and J. P. Heritage, "Cavity length detuning characteristics of the synchronously mode-locked $\mathrm{cW}$ dye laser," IEEE J Quant. Electron. QE-15, 915-917 (1979).

[13] D. M. Kim, J. Kuhl, R. Lambrich and D. von der Linde, "Characteristics of picosecond pulses generated from synchronously pumped cw dye laser system," Opt. Comm., 27, 123-126 (1978).

[14] Z. A. Yasa and O. Teschke, "Picosecond pulse generation in synchronously pumped dye lasers," Opt. Comm., 15, 169-172 (1975).

[15] A. Scavennec "Mismatch effects in synchronous pumping of the continuously operated mode-locked dye laser," opt. comm., 17, 14-17, (1976).

[16] J. Herrmann and U Motschmann, "Formation of synchronously mode-locked dye laser pulses," opt. Comm., 40, 379-384, (1982).

[17] L. M. Davis, J. D. Harvey and J. M. Peart, "Rate equation simulation of a synchronously pumped dye laser," Opt. Comm., 50, 49-55 (1984).

[18] T. Urisu and Y Mizushima, "Transient pulse buildup mechanisms in a synchronously-pumped mode-locked dye laser," J. Appl. Phys., 57, 1518-1522 (1985).

[19] J. J. Kasinski, L. A. Gomez-Jahn, R. J. D. Miller, P. Geist, B. Geoffrey, F. Heisel, A. Martz and J. A. Miehe, "Picosecond pulse formation in synchronously pumped dye lasers," J. Opt. Soc. Am. B, 3, 1566-1572 (1986).

[20] J. M. Catherall and G. H. C. New, "Role of spontaneous emission in the dynamics of mode locking by synchronous pumping," IEEE J. Quant. Electron., QE-22, 1593-1599 (1986).

[21] U. Stamm, "Numerical analysis of pulse generation in synchronously mode-locked cw dye lasers," Appl. Phys. B 45, 10i-108 (1988). 
[22] V. A. Nehaenko, Theory of mode-locked pumping of dye lasers," Sov. J. Quant. Electron., 11, 446-449 (1981).

[23] A. I. Kovrigin, V. A. Nekhaenko, S. M. Pershin and A. A. Podshivalov, "Dynamics of emission from dye lasers pumped synchronously by finite picosecond pulse trains," Sov. J. Quant. Electron., 14, 1346-1353 (1984).

[24] L. W. Casperson, "Coherence effects in synchronously pumped mode-locked dye lasers," J. Appl. Phys., 54, 2198-2208, (1983).

[25] Bahram Zandi, Lee W. Casperson and D. L. MacFarlane, "Experimental verification of a semiclassical model for synchronously pumped mode-locked dye lasers with bandwidth limiting elements," Proceedings of the 14th Congress of the International Comission for Optics, Quebec, Canada, August, 1987.

[26] J. P. Heritage and R. K. Jain, "Subpicosecond pulses from a tunable cw mode-locked dye laser," Appl. Phys. Lett. 32, 101-103 (1978).

[27] D. L. MacFarlane and L. W. Casperson, "Pump pulse effects in synchronously pumped mode-locked dye lasers," J. Opt. Soc. Am. B, 6, $292-299$ (1989).

[28] R. W. Hellwarth, "Theory of the pulsation of flourescent light from ruby," Phys. Rev. Lett., 6, 9 - 12 (1961).

[29] A. A. Vuylsteke, "Theory of laser regeneration switching," $\mathrm{J}$. Appl. Phys., 34, 1615 - 1622 (1963).

[30] W.G. Wagner and B. A. Lengyel, "Evolution of the giant pulse in a laser," J. Appl. Phys., 34, 1615 - 1622 (1963).

[31] C. C. Wang, "Optical giant pulses from a Q-switched laser," Proc. IEEE, 51, 1767 (1963).

[32] L. W. Casperson, M. J. Herbst and John J. Turechek, "Pulse evolution in $\mathrm{CO}_{2}$ lasers," J. Appl. Phys., 47, 5099 - 5101 (1976).

[33] K. C. Reyzer and L. W. Casperson, "Polarization characteristics of dye-laser amplifiers II. Isotropic molecular distributions," J. Appl. Phys., 51, 6083 - 6090 (1980). 
[34] E. N. Lorenz, "Deterministic non-periodic flows," J. Atmos. Sci., $20,130-141(1960)$.

[35] C. Sparrow, The Lorenz Equations: Bifurcations, Chaos and Strange Attractors, Springer-Verlag, Berlin, 1982.

[36] H. Haken, "Analogy between higher instabilities in fluids and lasers," Phys. Lett., 53A, 77 - 78, (1975).

[37] P. W. Milonni, R. B. Gibson and A. J. Taylor, "Ultrashort pulse propagation in $\mathrm{KrF}$ laser amplifiers," J. Opt Soc. Am B, 5, $1360-$ 1368 (1988).

[38] H. B. Dwight, Tables of Integrals and Other Mathematical Data, Macmillan Publishing Co. Inc., New York, 1961, p38, \#160.01.

[39] H. A Haus, C. V. Shank and E. P. Ippen, "Shape of passively mode-locked laser pulses," Opt. Comm. 15, 29 - 31 (1975).

[40] S. L. McCall and E. L. Hahn, "Self-induced transparency by coherent light," Phys Rev. Lett. 18, 908 - 911 (1967).

[41] S. L. McCall and E. L. Hahri, "Self-induced transparency," Phys. Rev. 183, $457-485$ (1969).

[42] S. L. McCall and E. L. Hahn, "Pulse-area-pulse-energy description of a traveling-wave laser amplifier," Phys. Rev. A 2, $861-870$ (1970).

[43] R. L. Fork, B. I. Greene and C. V. Shank, "Generation of optical pulses shorter than 0.1 psec by colliding pulse mode-locking," Appl. Phys. Lett., 38, 671 - 672, (1981).

[44] W. Dietal, "Transient absorber gratings shorten the pulses of a passively mode-locked cw dye laser," Opt. Comm. 43, 69-71 (1982).

[45] G. W. Fehrenbach, K. J. Gruntz and R. G. Ulbrich, "Subpicosecond light pulses from a synchronously mode-locked dye laser with composite gain and absorber medium," Appl. Phys. Lett., 33, 15\% 160, (1978)

[46] G. A. Mourou and T. Sizer II, "Generation of pulses shorter than $70 \mathrm{fs}$ with a synchronously-pumped cw dye laser," Opt. Comm., $41,47-48,(1982)$ 
[47] J. A. Valdmanis, R. L. Fork and J. P. Gordon, "Generation of optical pulses as short as 27 femtoseconds directly from a laser balancing self-phase modulation, group velocity dispersion, saturable absorption and saturable gain," Opt. Lett. 10, 131 - 133 (1985).

[48] M. D. Dawson, T. F. Boggess and D. W Garvey, "A hybridly mode-locked $\mathrm{CW}$ dye laser with Brewster prisms," Opt. Comm. 60, $79-83$ (1986).

[49] M. D. Dawson, T. F. Boggess, D. W Garvey and A. L. Smirl, "Femtosecond pulse generation in the red/deep red spectral region," IEEE J. Quant. Electron. QE-23, 290-292 (1987).

[50] M. Nakazawa, T. Nakashi, H. Kubota and S. Seikai, "65-femtosecond pulse generation from a synchonously pumped dye laser without a colliding-pulse mode-locking technique," Opt. Lett. 12, $681-683$ (1987).

[51] J. P. Ryan, L. S. Goldberg and D. J. Bradley, "Comparison of synchronous pumping and passive mode-locking of $\mathrm{CW}$ dye lasers for the generation of picosecond and subpicosecond light pulses," Opt. Comm. 27, 127 - 132 (1987).

[52] M. D. Dawson, T. F. Boggess and A. L. Smirl, "Femtosecond synchronously pumped pyridine dye isers," opt. Lett. 12, 254 256 (1987).

[53] M. D. Dawson, D. Maxson, T. F. Boggess and A. L. Smirl, "Cavity-length detuning effects and stabilization of a synchronously pumped femtosecond linear dye laser," Opt. Lett. $13,126-128$ (1988).

[54] D. Kuhlke, U. Herpers and D. von der Linde, "Characteristics of a hybridly mode-locked cw dye laser," Appl. Phys. B 38, $233-240$ (1985).

[55] J. M. Catherall and G.H. C. New, "Theoretical studies of active, synchronous and hybrid mode-locking," in Ultrafast Phenomena IV, ed. by D. H. Auston and K. B. Eisenthal, Springer Verlag, Berlin (1984) pp $75-77$ 
[56] Kh. G. Gafurou, D. P. Kindach, V. A. Nekhaenko and A. G.

Yakovlev, "Enhancement of the stability of a synchronously excited $\mathrm{cw}$ dye laser by insertion of a nonlinear absorber," Sov. J. Quantum. Electron. 15, 844 - 846 (1985).

[57] G. H. C. New and K. E. Aldous, "Problems in implementing the self-consistent profile technique in mode-locking theory," IEEE $J$. Quant. Electron. 24, 2288 - 2293 (1988).

[58] W. E. Lamb, Jr. "Theory of an optical maser," The Phys. Rev. 134, A1429 - A1450 (1964).

[59] K. C. Reyzer and L. W. Casperson, "Polarization characteristics of dye-laser amplifier I. Unidirectional molecular distributions, "J. Appl. Phys. 51, $6075-6082$ (1980).

[60] R. L. Fork, C. V. Shank and R. T. Yen, "Amplification of 70 fs optical pulses to gigawatt powers," Appl. Phys. Lett. 41, $223-225$ (1982).

[6]] W. H. Knox, M. C. Downer, R. L. Fork and C. V. Shank, "Amplified femtosecond optical pulses and continuum generation at a $5-\mathrm{kHz}$ repetition rate," Opt. Lett. 9, 552 - 554 (1984).

[62] Y. Ishida, T. Yajima and K. Naganuma, "Generation of broadly tunable subpicosecond light pulses from a synchronoulsy and passively mode-locked cw dye laser," Jap. Journ. Appl. Phys. 19, L717 - L720 (1986)

[63] D. Ricard and $J$. Ducuing, "Vibrational relaxation in the ground and first excited electronic states of large organic molecules in solution," IEEE J. Quant. Electron. QE-10, 745 - 746 (1974).

[64] D. Ricard and J. Ducuing, "Vibrational relaxation in the ground electronic states of large organic molecules in solution," J. Chem. Phys. 62, 3616 - 3619 (1975).

[65] A. Penzkofer, W. Falkenstein and W. Kaiser, "Vibronic relaxation in the $S_{1}$ state of rhodamine dye solutions," Chem. Phys. Lett. $44,82-87$ (1976).

[66] T. Yajima, H. Souma and Y. Ishida, "Study of ultrafast relaxation processes by resonant Rayleigh-type optical mixing. II Experiment on dye solutions," Phys. Rev. A 17, 324 - 334 (1978). 
[67] M. M. Malley and G. Mourou, "The picosecond time-resolved fluorescence spectrum of rhodamine 6G," Opt. Comm. 10,323326. (1974).

[68] B. K. Garside and T. K. Lim, "Ultrashort pulses from mode-locked Cw dye lasers," Opt. comm. 8, 297 - 301 (1973).

[69] B. K. Garside and T. K. Lim, "Passive mode-locking in flashlamp-pumped dye lasers," Opt. Comm. 12, 240 - 245 (1974).

[70] D. N. Dempster, T. Morrow, R. Rankin and G. F. Thompson, "Photochemical characteristics of cyanine dyes," J. Chem. Soc. Faraday II 68, 1479 - 1496 (1972).

[71] E. G. Arthurs, D. J. Bradley and A. G. Roddie, "Picosecond measurements of $3,3^{\prime}$ diethyloxadicarbocyanine iodide and photoisomer fluorescence," Chem. Phys. Lett. , 230 - 234 (1974).

[72] D. Magde and M. W. Windsor, "Picosecond flash photolysis and spectroscopy: 3,3' diethyloxadicarbocyanine iodide (DODCI)," Chem Phys Lett. 27, 31 - 36 (1974).

[73] G. E. Busch, K. S. Greve, G. L. Olson, R. P. Jones and R. M. Rentzepis, "Photobleaching and recovery times of the mode-locking dye DODCI," Chem. Phys. Lett. 33, 412 - 416 (1975).

[74] J. Jaraudias, P. Goujon and J. C. Mialocq, "Picosecond absorption relaxation of DODCI using single pulse excitation," Chem. Phys. Lett. $45,107-110$ (1977).

[75] T. Yajima, Y. Ishida and Y. Taira, "Investigation of subpicosecond dephasing processes by transient spatial parametric effect in resonant media," in Picosecond Phenomena II ed. by $R$. Hochstrasser, W. Kaiser and C. V. Shank, Springer Verlag, Berlin, 1980 , pp $190-194$.

[76] J. J. Song, J. H. Lee and M. D. Levenson, "Picosecond relaxation measurements by polarization spectroscopy in condensed phases," Phys. Rev. A 17, $1439-1447$ (1978).

[77] P. Beaud, B. Zysset, A. P. Schwarzenbach and H. P. Weber, "1.3 um subpicosecond pulses from a dye laser pumped by compressed Nd:YAG - laser pulses," Opt. Lett. 11, 24 - 26 (1986). 
[78] L. E. Hargrove, R. L. Fork and M. A. Pollack, "Locking of HeNe laser modes by synchronous intracavity modulation," Appl. Phys. Lett., 5, 4- 5, (1964).

[79] P. M. W. French, A. S. L. Gomes, A. S. Gouveia-Neto and J. R. Taylor, "Spectral optimisation of a synchronousiy mode-locked femtosecond dye laser," Optics Comm., 60, 389 - 392, (1986).

[80] G. H. C. New and J. M. Catherall, "Perturbations \& instabilities in laser mode-locking dynamics," in optical Instabilities, $\mathrm{R}$. W. Boyd, M. G. Raymer \& L. M. Narducci, eds., Cambridge: Cambridge University Press, 1986, pp 196 - 211.

[81] B. Xiaoyi and W. Cunkai, "Stability criteria for pulse solution of a synchronously pumped mode-locked dye laser," Optics Lett., 12,251 - 253, (1987).

[82] J. Kluge, D. Wiechert and D. Von der Linde, "Fluctuations in synchronously mode-locked dye lasers," Optics Comm.,51, 271 - 277, (1984).

[83] S. L. Shapiro, R. R. Cavanagh and J. C. Stephenson, "Streak-camera observations of the pulse emission from a synchronously pumped continuous-wave mode-locked dye laser." Optics Lett., 6, 470 - 472 (1981).

[84] T. M. Baer and D. D. Smith, "Noise in picosecond laser systems: Actively mode locked $\mathrm{CW} \mathrm{Nd^{3+ }}$ Y $\mathrm{AG}$ and $\mathrm{Ar}^{+}$lasers synchronously pumping dye lasers," in Ultrafast Phenomena IV D.H. Auston \& K. B. Eisenthal, eds., Berlin: Springer Verlag, 1984, pp $96-98$.

[85] J. P. Zheng, U. Sen, D. M. Benenson and H. S. Kwok, "Observation of periodicity multiplication in a synchronously pumped dye laser," Optics Lett., 11, 632 - 634, (1986).

[86] D. L. MacFarlane and Lee W. Casperson, "Pulse train instabilities in a mode-locked argon laser," J. Opt. Soc. Am B 4, 1777 - 1780 (1987).

[87] L. W. Hillman, J. Krasinski, R. W. Boyd and C. R. Stroud, Jr., "Observation of higher order dynamical states of a homogeneously broadened laser," Phys. Rev. Lett., 52, 1605-1608 (1984). 
[88] N. M. Lawandy, R. Sohrab Afzal and W. S. Rabinovich, "Bichromatic emission in a ring dye laser," Phys. Rev. A, 36, 1759-1770, (1987).

[89] T. Ogawa and E. Hamamura, "Dynamical properties of the multimode laser with modulated inversion," Optics. Comm., 61, 49-54 (1987).

[90] Fu Hong and H. Haken, "A band model for dye lasers and the unidirectional single-frequency operation," presented at the International Workshop on Instabilities, Dynamics and Chaos in Nonlinear Optical Systems, Lucca, Italy, July 8-10, 1987.

[91] O. P. MCDuff and S. E. Harris, "Nonlinear Theory of the Internally Loss-Modulated Laser," IEEE U. Quant. Electron., vol QE-3, pp 101 - 111, (1967).

[92] S. E. Harris and O.P. McDuff, "Theory of FM Laser Oscillation," IEEE J. Quant Electron., vol QE-1, pp245 - 262 (1965).

[93] A.E. Siegman and D. J. Kuizenga " Modulator Frequency Detuning Effects in the FM Mode-Locked Laser," IEEE J. Quant. Electron., vol QE-6, pp $803-808$, (1979).

[94] L. W. Casperson and A. Yariv, "The Time Behavior \& Spectra of Relaxation Oscillations in a High Gain Laser," IEEE J. Quant. Electron., vol QE-8, pp $69-73$, (1972).

[95] T. Mukai and K. Otsuka, "Subharmonic Oscillations and Chaos in a Laser Diode Coupled to an External Cavity," Optical Instabilities, ed by R. W. Boyd, M.G. Raymer \& L. M. Narducci, Cambridge Univ. Press, pp $72-84,(1986)$.

[96] B. Van der Pol and J. Van der Mark, "Frequency Demultiplication," Nature, vol 120, 363 - 364 (1927).

[97] M.J. Feigenbaum, "Universal Behavior in Nonlinear Systems," Los Alamos Science, vol 1, 4-27, (1980).

[98] C. Sparrow, "The Lorenz Model" Optical Instabilities, ed by R. W. Boyd, M.G. Raymer \& L. M. Narducci, Cambridge Univ. Press, 72-84, (1986). 
[99] L.W. Casperson, "Spontaneous Coherent Pulsations in Ring-Laser oscillators," J. Opt Soc. Am B, vol 2, 62 - 72, (1985).

[100]L. W. Casperson, L.M. Davis, and J. D. Harvey, "Thermal Hysteresis in Acoustic Resonators," J. Acoust. Soc Am., vol 71, 1412 - 1416, (1982).

[101] Paul S. Linsay, "Period Doubling and Chaotic Behavior in a Driven Anharmonic Oscillator," Phys. Rev. Lett., vol. 47, 1349 1352, (1981). 


\begin{abstract}
APPENDIX
PULSE TRAIN INSTABILITIES IN A MODE-LOCKED ARGON LASER: EXPERIMENTAL STUDIES
\end{abstract}

INTRODUCTION

Since the first reports of active mode-locking in lasers over two decades ago [78], this technique of producing a perfectly repetitive train of ultrashort pulses has been developed into a routine mode of operation for many commercial laser systems. Since many mode-locking applications demand pulse-to-pulse consistency, much progress has been made in avoiding unpredictable and irregular mode-locked laser outputs in loss modulated lasers. Meanwhile, work in the theoretical arena has focussed on similar goals and several general models now exist that are valid and useful for optimizing actively mode-locked laser operation $[9,91-93]$. In spite of these efforts there remain several types of mode-locked lasers in which the observed pulsations are not exactly periodic and have not yet been rigorously interpreted in terms of of a theoretical model. In the case of self-locked xenon lasers, for example, the slow chaotic envelope modulation that is sometimes observed [94] has not yet been modeled. Irregular pulse trains and subharmonic phenomena have also been seen in extended cavity semiconductor lasers [95] and in actively mode-locked lasers such as synchronously pumped dye lasers $[15,82,85]$ when the modulation frequency is not properly matched 
to the pulse repetition frequency. These effects in driven laser oscillators are analogous to the chaotic behavior of driven electronic oscillators first studied by Van der Pol and Van der Mark [96]. Our understanding of the synchronous gain or loss modulation of a laser cannot be considered complete until the structure of such imperfect mode-locking is understood.

With the above in mind, we have carried out a systematic experimental study of an imperfectly mode-locked laser. In particular, we report the effect of small detunings of the modulation frequency on the output pulse train of an acousto-optically mode-locked argon laser. The quantities studied include the peak and average powers, the energy per pulse, and the temporal pulse shape. Additionally, the character of the pulse train was monitored using a real time oscilloscope and a homodyne spectrum analyzer. From this data, the different phases of operation can be classified with respect to the modulation frequency. We find that decreases in the modulation frequency of as little as one part in $10^{4}$ produce strong lower frequency components that modulate the amplitude of the train of ultrashort pulses. The strongest of these components has a frequency of about $20.5 \mathrm{MHz}$, which is close to the period-four [97] frequency of $\mathrm{c} / 2 \mathrm{~L}$, which is approximately $81 \mathrm{MHz}$. As the modulaticn frequency is further decreased, a slow amplitude envelope of several hundred $\mathrm{KHz}$ develops on the pulse train. At first this amplitude modulation is fairly regular in frequency, but upon further detuning, the modulation depth approaches unity and all frequencies less than about $600 \mathrm{KHz}$ are represented. This filling in of the homodyne power spectrum is also found on the immediate high side of the optimum modulation frequency, and is characteristic of other deterministically chaotic systems $[97,98]$. 
Notable too is the increase in average power when a new frequency component appears. Similar increases in output power at phase transitions have been noted in previous studies of laser instabilities [99].

\section{EXPERIMENTS}

The experiments were performed using a commercially available Spectra Physics 2020 argon ion laser with a $186 \mathrm{~cm}$ long stable resonator. The laser was operated in its TEM 00 transverse mode. A fused silica acousto-optic modulator positioned adjacent to the high reflectivity mirror was driven at approximately $40 \mathrm{MHz}$ to mode-lock the laser. This prism also served to limit laser oscillation to the $514 \mathrm{~nm}$ line. A typical average output power for this system is $400 \mathrm{~mW}$ as measured with a Scientech 362 calorimeter.

The beam was sampled using two fast Si PIN diodes (Antronics S-2), operating photoconductively with risetimes of less than 35 psec. One of the signals was fed to a Tektronix 7L12 spectrum analyzer in a 7613 mainframe. This setup allowed constant monitoring of the homodyne power spectrum as a function of the modulation frequency. The second photodiode was used to study the temporal output. The pulse train was monitored using a Tektronix 7834 storage scope which was essential in viewing some of the irregular (non-triggerable) signals. A composite pulse was also constructed using a Tektronix 7Sll sampling plug-in with an S-4 head. This system allowed an accurate representation of the 100 psec mode-locked pulses.

The acousto-optic prism was driven by a standard Spectra 
Physics $452 \mathrm{~A}$ mode-locker driver with a 453 stabilizing unit. Our control parameter was the modulation frequency which was controllable to a resolution of $<200 \mathrm{~Hz}$, or about 2 parts in $10^{5}$. Since a standing acoustic wave builds in the prism, this frequency is exactly half of the modulation frequency of the laser. The mode locker was kept in thermal equilibrium at all times in this experiment [100].

Since this experiment studies the frequency content of the acousto-optic modulator/argon laser system, it was important to directly determine the frequencies present in the acousto-optic modulator alone, and thereby to ensure that the new frequency content did not arise solely from some anomaly in the acoustooptic modulator. This characterization was done in several ways. Most directly, a HeNe alignment laser was used to probe the oscillating prism over the driving range of the Ar laser experiments. The HeNe beam was directed into the prism, off the high reflectivity mirror and back out through the prism. This output was directed into one of the fast photodiodes, and the induced modulation was studied using a real time oscilloscope and a spectrum analyzer. Aside from the fundamental $81 \mathrm{MHz}$ modulation, there were no frequency components commensurate with the subharmonic frequencies present in the Ar laser experiment. In this manner, the modulation depth impressed on the laser beam was measured to be about 608 at an $\mathrm{rf}$ power of 0.9 Watts. Additionally, no frequencies of interest in the $\mathrm{Ar}$ system were found on the driving voltage of the acousto-optic crystal when that was monitored, and no high frequency components were observed on the Ar discharge voltage. 
RESULTS

Figures $35 a-35 d$ are sampling scope composites of the mode-locked pulse at various phases of laser operation determined by frequency detuning, which we define as,

$$
\delta=\left(f_{\text {opt }}-\mathrm{f}\right) / \mathrm{f}_{\text {opt }} .
$$

Even though these traces represent the superposition of many pulses which at times in this experiment were not identical, much can still be inferred from them. Figure 35a represents an optimized ultrashort laser pulse emitted when the acousto-optic modulator is driven at $\mathrm{f}_{\text {opt }}$. Figure $35 \mathrm{~b}$ shows the pulse at a modulator frequency which is $2.5 \mathrm{kHz}$ below $\mathrm{f}_{\text {opt }}$, a detuning of $\delta=$ $6.1 \times 10^{-5}$. The output now consists of a significant second pulse trailing the original by 450 psec. It is this pulse which is modulated with any further detuning. This $\delta$ is the threshold for new frequency components. The effect of this modulation is shown in figure $35 \mathrm{c}$, taken at $\delta=12.2 \times 10^{-5}$. The spread in the amplitude of the second pulse is interpreted as a fast change in intensity from one pulse to another. The character of this pulse-to-pulse variation can be found from spectrum analyzer and real time integrated pulse measurements. From these we determine that the second pulse is varying regularly at a frequency of $20.5 \mathrm{MHz}$. Agreement in the modulation depths calculated from all three types of data corroborates this interpretation.

A second phenomenology occurs at a $\delta$ of $15.9 \times 10^{-5}$. The sampling scope trace of figure 35c appears to be just an extreme 
case of $35 \mathrm{c}$, but while there is a strong $20.5 \mathrm{MHz}$ frequency component still present, there is also a slow (100-600 kHz) modulation of nearly $75 \%$. Moreover, as the detuning is increased, this envelope becomes more irregular and the initially clean frequency spectrum fills in. Figures $36 \mathrm{a}$ and $36 \mathrm{~b}$ show the slow envelope at detunings of $19.6 \times 10^{-5}$ and $22.0 \times 10^{-5}$ respectively. At a time base of a few microseconds per division, the $81 \mathrm{MHz}$ pulse train is unresolved, but present. In figures $37 \mathrm{a}$ and $37 \mathrm{~b}$ are the laser power spectra about $81 \mathrm{MHz}$ corresponding to the detunings of figure 36 . Of note in figure $37 \mathrm{~b}$ is the lifted baseline indicating that all frequencies less than approximately $400 \mathrm{kHz}$ are present.

Interestingly, the phenomenology described above are not mirrored when the modulation frequency is greater than the optimum. Instead we see a rapid degradation in lasing output as the modulation frequency increases. Additionally, the ultrashort pulses become chaotic almost immediately. There is no second pulse development, however there is the same erratic, slow envelope shown in figures $36 \mathrm{~b}$ and $37 \mathrm{~b}$.

The transitions between the three phases of operation described here can also be illustrated by studying the average power as a function of detuning. Such a plot is shown in figure 38 . of interest are the increases in power at $\delta=6.1 \times 10^{-5}$, the transition point for the $20.5 \mathrm{MHz}$ modulation, and at $\delta=16 \times 10^{-5}$, the onset of the slow modulation at several hundred $\mathrm{kHz}$.

\section{DISCUSSION}

We have studied the output of an imperfectly mode-locked Ar 
laser in the regime of small detunings from the optimum loss modulation frequency. We have observed a change in the temporal shape of the pulse with the onset of an "echo" pulse that lags the original pulse by $450 \mathrm{psec}$. With further detuning, the energy in this second pulse is modulated, first at a frequency approximately equal to $\mathrm{f}_{\mathrm{opt}} / 4$ and later at much slower frequencies $\left(10^{5} \mathrm{~Hz}\right)$. This slow modulation is initially a fairly clean amplitude modulation, however with further detuning, this envelope becomes chaotic as more sideband frequencies appear.

A heuristic argument may be formulated to explain at least the $\mathrm{f} / 4$ bifurcation. This picture treats the laser as a loss-driven oscillator with a nonlinearity due to a saturating gain. In inhomogeneous lasers, the gain saturates as $(1+\mathrm{sl})^{-1 / 2}$. This form is similar to the nonlinearity found in varactor diodes and a rich array of subharmonic bifurcation schemes have been observed in RLC/varactor diode circuits [101]. Preliminary theoretical work to explain mode-locking irregularities as a consequence of nonlinear laser dynamics is now underway. 


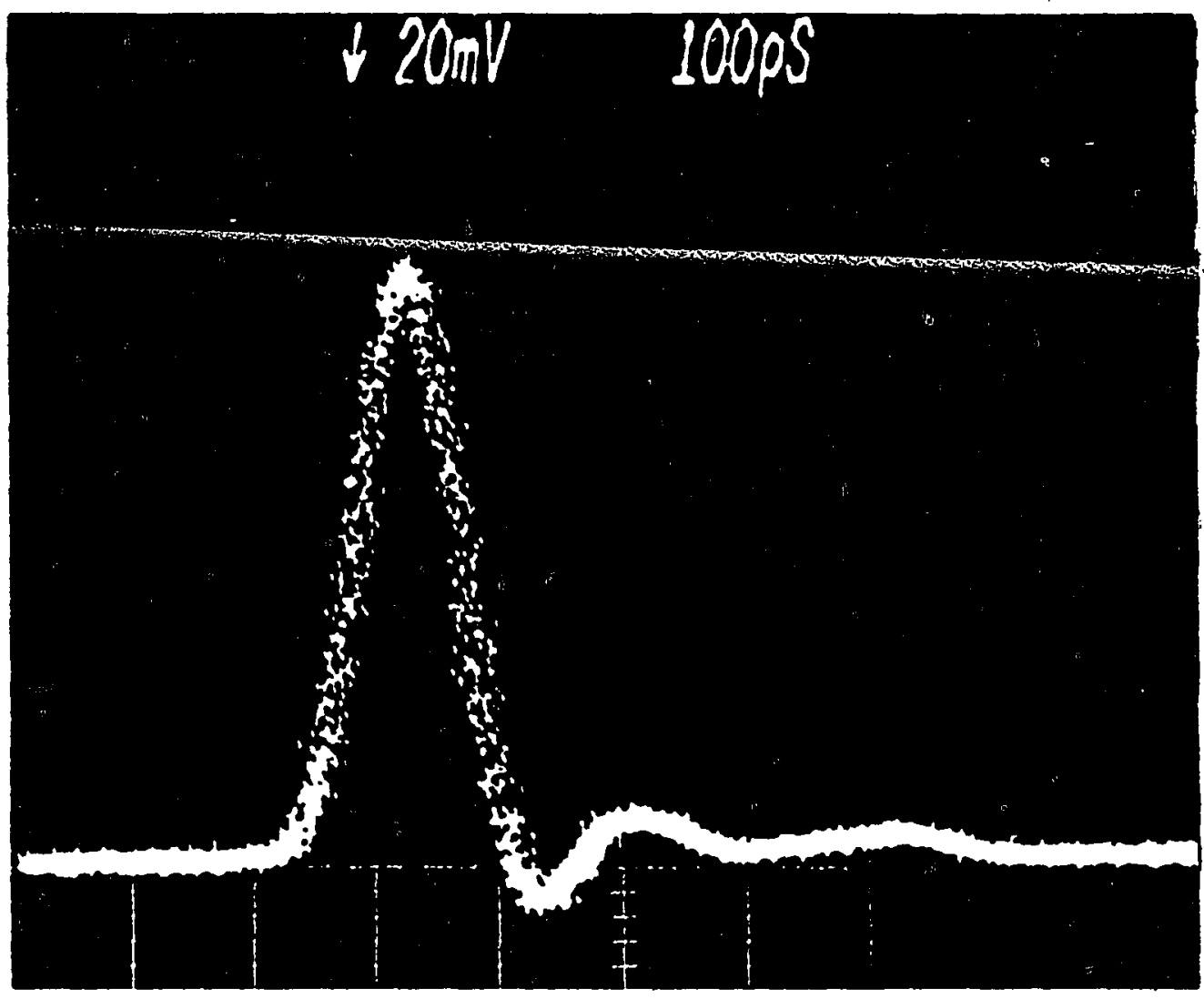

Figure 35a. Sampling scope trace. $\delta=0$. 


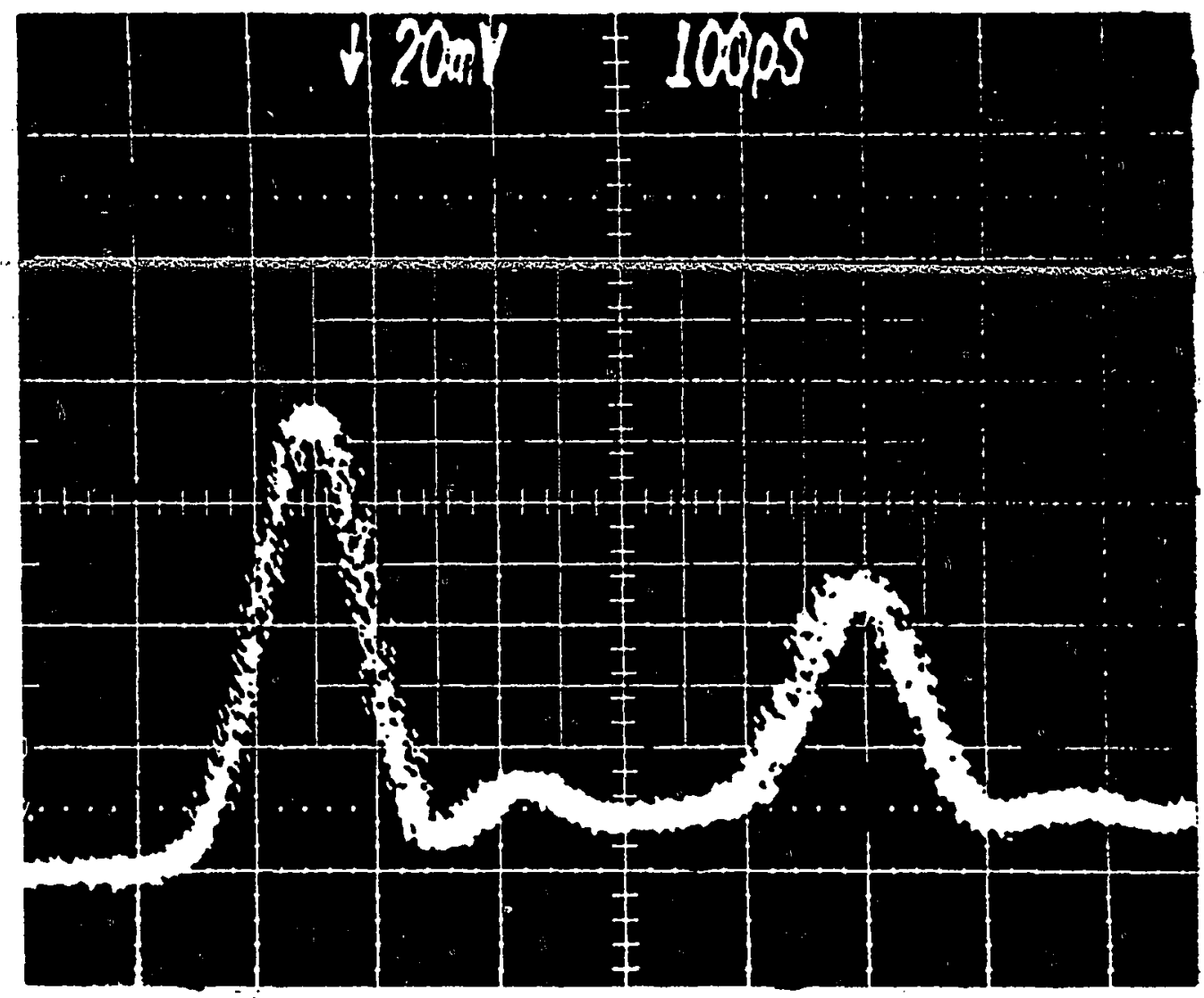

Figure 35b. Sampling scope trace. $\delta=6.1 \times 10-5$ 


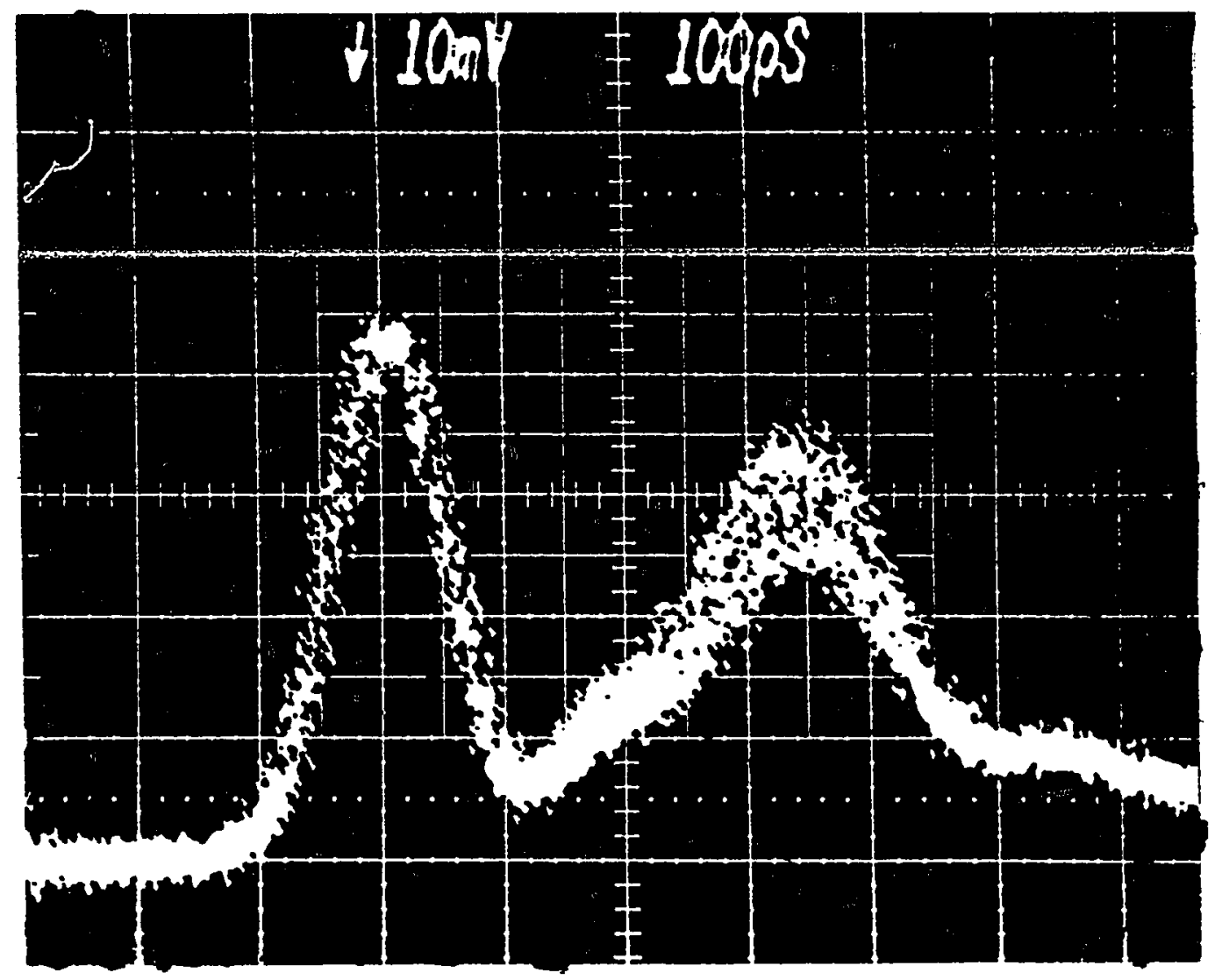

Figure 35c. Sampling scope trace. $\delta=12.2 \times 10^{-5}$ 
159

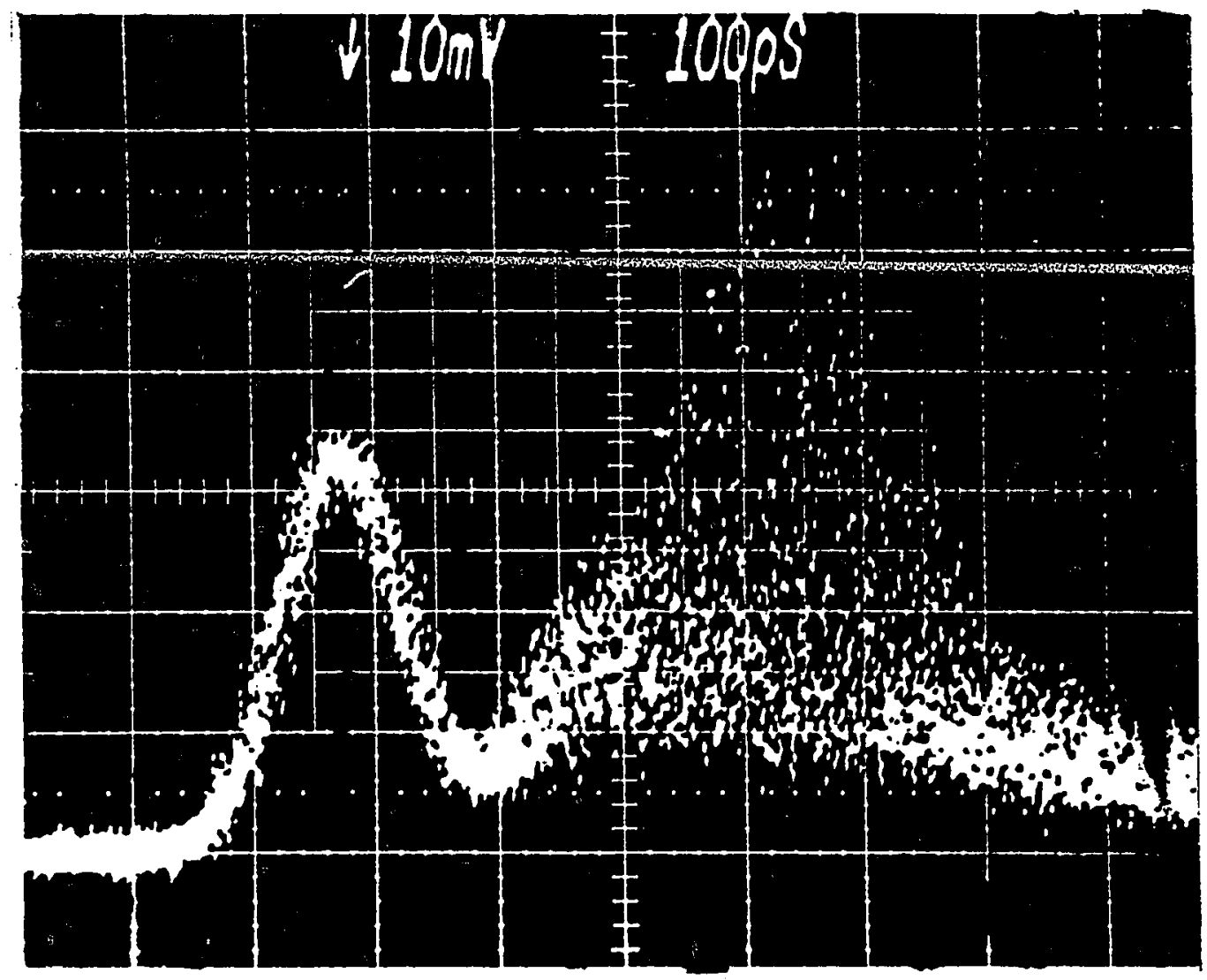

Figure 35d. Sampling scope trace. $\delta=15.9 \times 10^{-5}$ 


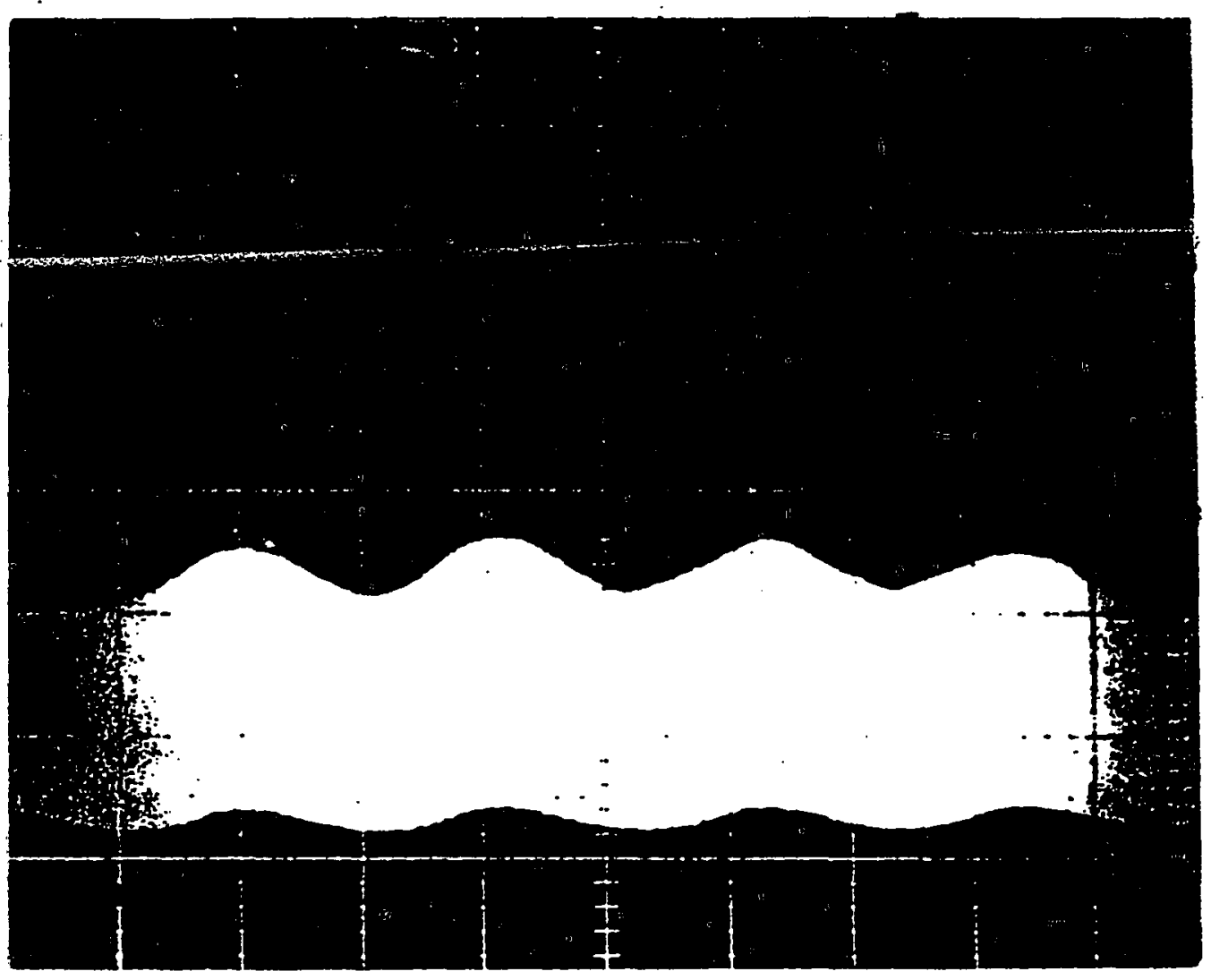

Figure 36a. Pulse train envelope. $\delta=19.6 \times 10^{-5}$ 


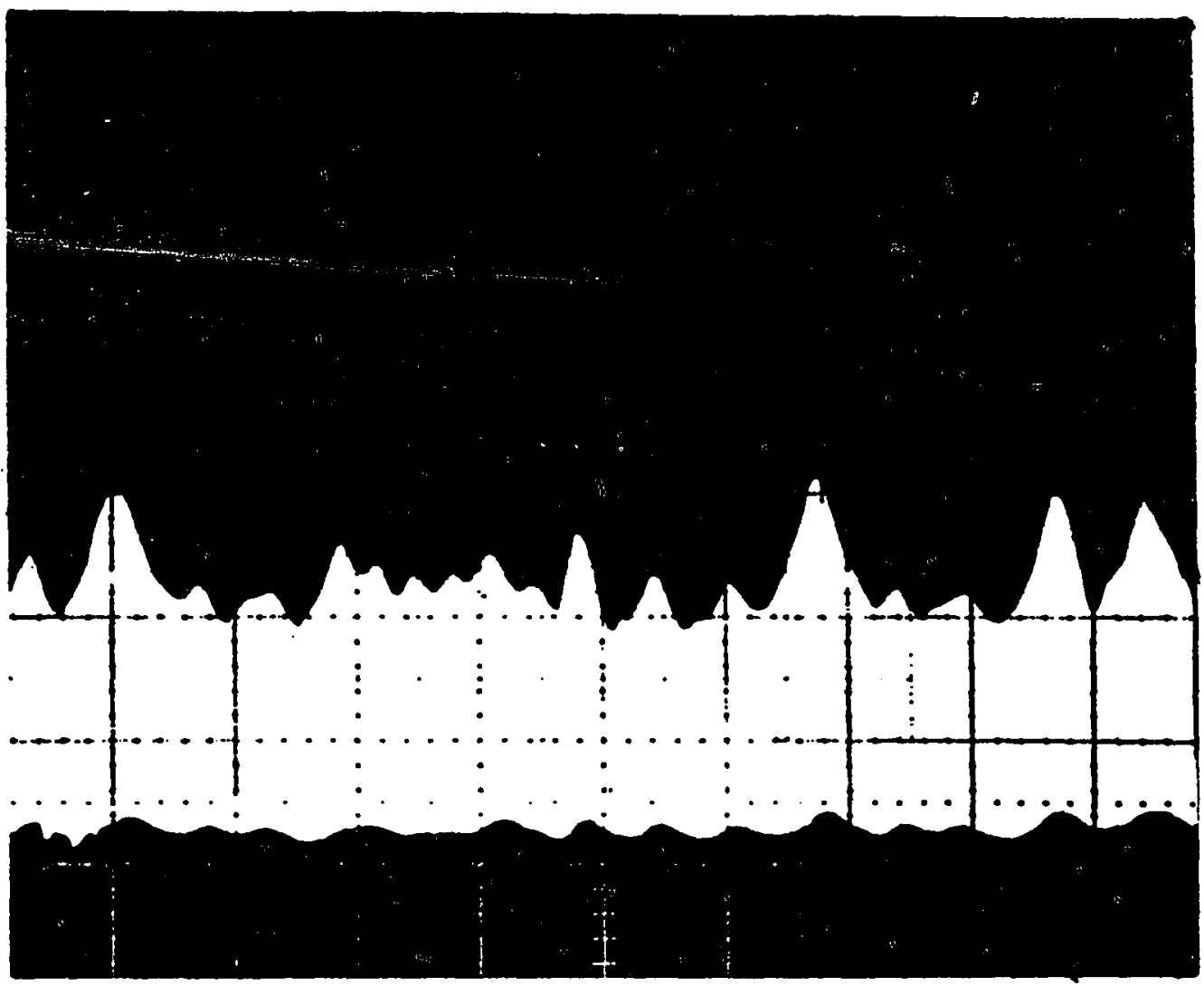

Figure 36b. Pulse train envelope. $\delta=22.0 \times 10^{-5}$ 


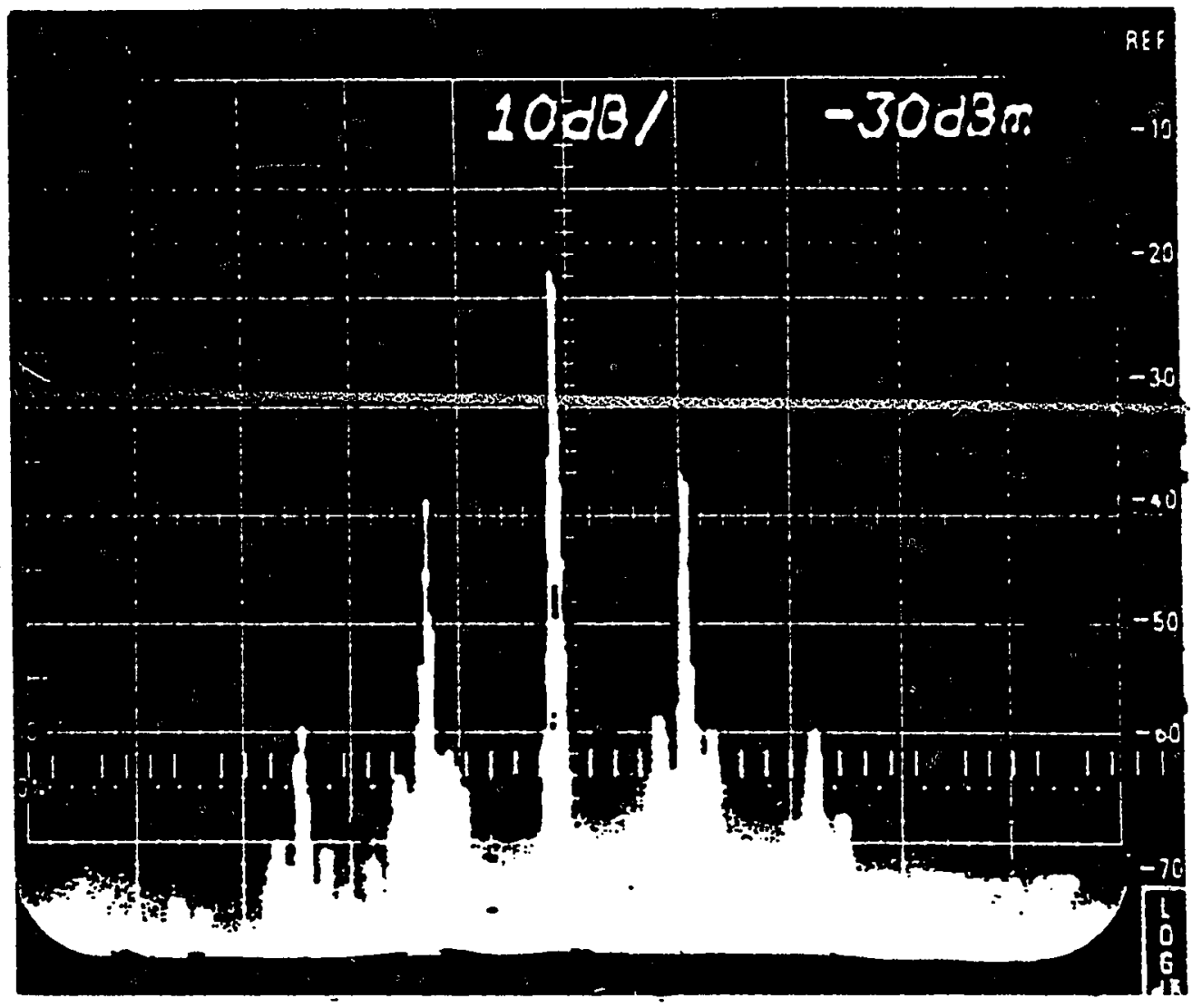

Figure 37 . If spectrum. $\delta=19.6 \times 10^{-5}$ 


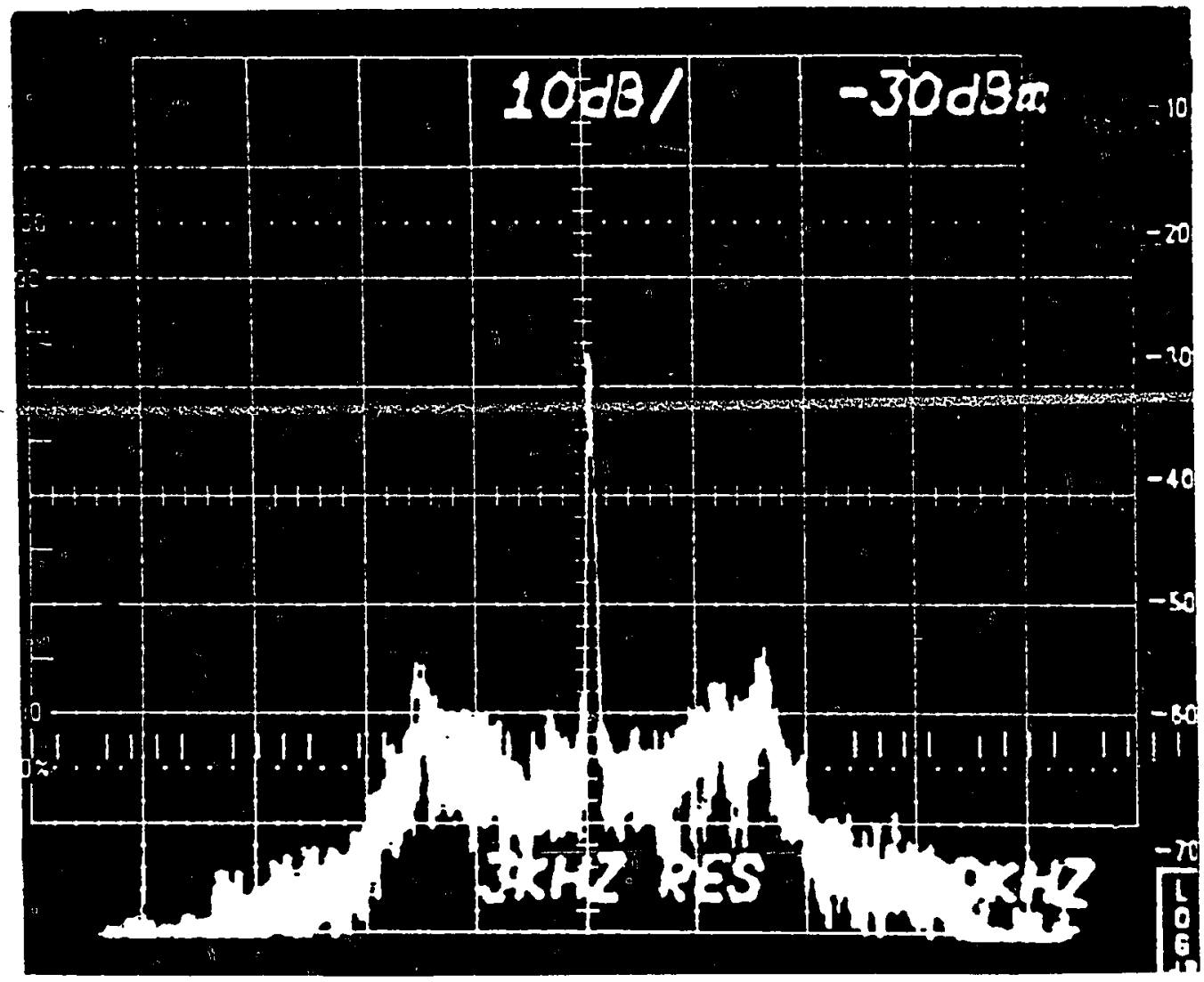

Figure $37 b$. if spectrum. $\delta=22.0 \times 10^{-5}$ 
164

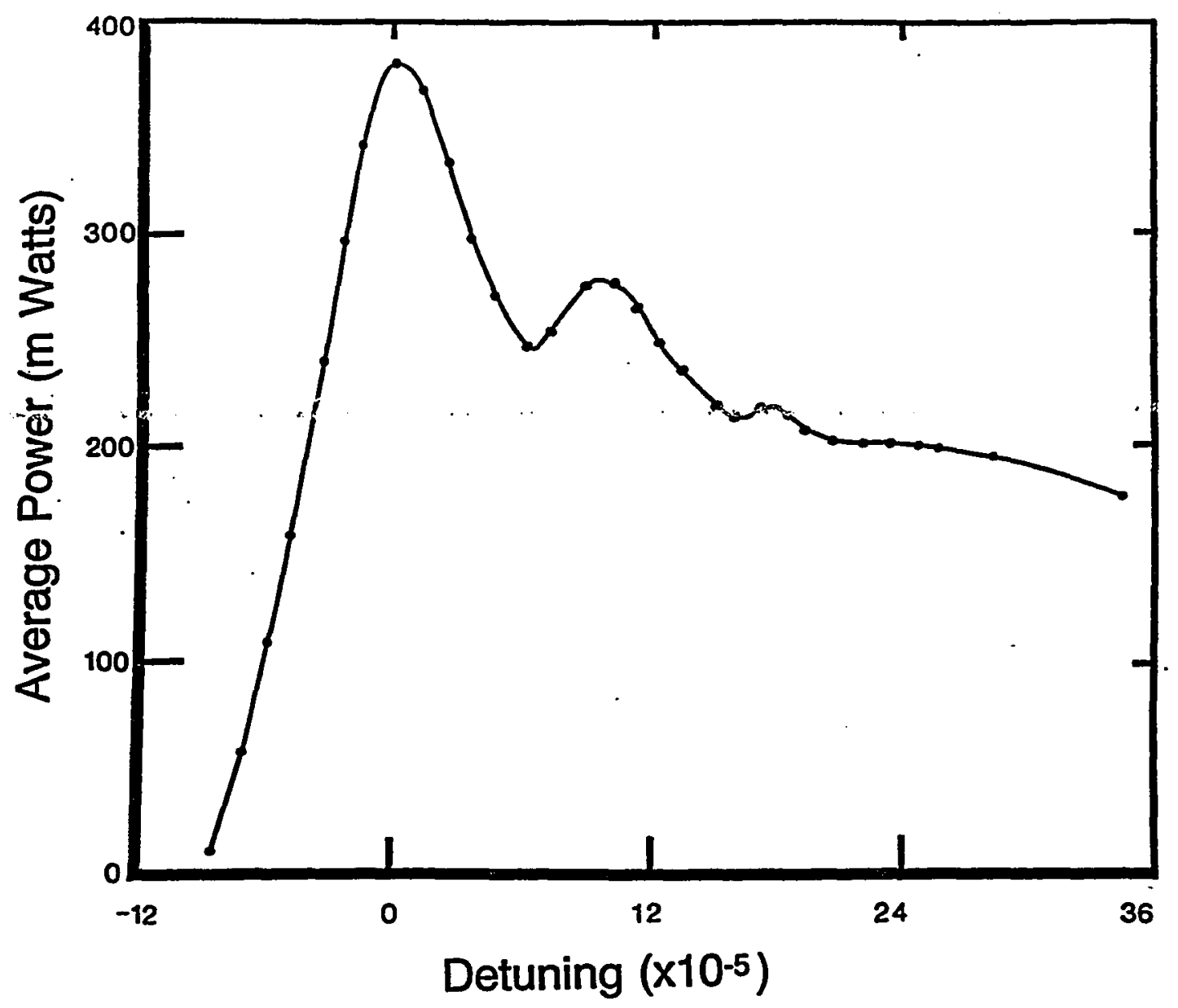

Figure 38. Average power versus detuning. 